\title{
Population
}

National..

Constituent countries of

the United Kingdom

Subnational Government Office Regions

Age and sex

Constituent countries of the United Kingdom

\section{Vital statistics}

Summary

Constituent countries of the United Kingdom ..Constituent countries of the United Kingdom

Key demographic and health indicators

\section{England and Wales}

\section{Live births}

Age of mother England and Wales

\section{Conceptions and abortions}

\section{Expectation of life}

\section{Deaths}

Age and sex the United Kingdom

Subnational. England and Wales ..Health Regional Office areas of England

\section{StatBase ${ }^{\circledR}$}

Health Statistics Quarterly tables are now available on StatBase ${ }^{\circledR}$ which can be accessed via our website www.statistics.gov.uk

\section{Symbols}

\author{
.. not available \\ not applicable \\ nil or less than half the final digit shown \\ blank not yet available
}




\section{Notes to tables}

\section{Time series}

For most tables, years start at 1971 and then continue at five-year intervals until 1991. Individual years are shown thereafter. If a year is not present the data are not available.

\section{United Kingdom}

The United Kingdom comprises England, Wales, Scotland and Northern Ireland. The Channel Islands and the Isle of Man are not part of the United Kingdom.

\section{Population}

The estimated resident population of an area includes all people who usually live there, whatever their nationality. Members of HM and US Armed Forces in England and Wales are included on residential basis wherever possible. HM Forces stationed outside England and Wales are not included. Students are taken to be resident at their term time addresses.

Further information on population estimates is available on the National Statistics website at: www.statistics.gov.uk/popest

\section{Live births}

For England and Wales, figures relate to the number of births occurring in a period; for Scotland and Northern Ireland, figures relate to births registered in a period. By law, births must be registered within 42 days in England and Wales, within 21 days in Scotland, and within 42 days in Northern Ireland. In England and Wales, where a birth is registered later than the legal time period, and too late to be included in the count for the year of occurrence, it will be included in the count for the following year.

\section{Perinatal mortality}

In October 1992 the legal definition of a stillbirth was changed, from a baby born dead after 28 completed weeks of gestation or more, to one born dead after 24 completed weeks of gestation or more.

\section{Period expectation of life}

The life tables on which these expectations are based use death rates for the given period to describe mortality levels for each year. Each individual year shown is based on a three-year period, so that for instance 1986 represents 1985-87. More information is available on the National Statistics website at: www.statistics.gov.uk/statbase/Product. asp?vlnk=14459

\section{Deaths}

Figures for England and Wales relate to the number of deaths registered in each year up until 1992, and the number of deaths occuring in a year between 1993 and 2005. From 2006 onwards, all figures relate to the number of deaths registered in the year. All figures for Scotland and Northern Ireland relate to the number of deaths registered in each year.

\section{Coding cause of death}

Between 1 January 1984 and 31 December 1992, ONS applied its own interpretation of the International Classification of Diseases (ICD) Section Rule 3 in the coding of deaths where terminal events and other 'modes of dying' such as cardiac arrest, cardiac failure, certain thrombembolic disorders, and unspecified pneumonia and bronchopneumonia, were stated by the certifier to be the underlying cause of death and other major pathology appeared on the certificate. In these cases ONS Rule 3 allowed the terminal event to be considered a direct sequel to the major pathology and that primary condition was selected as the underlying cause of death. Prior to 1984 and between 1 January 1993 and 31 December 2000, such certificates were coded to the terminal event. National Statistics also introduced automated coding of cause of death in 1993, which may also affect comparisons of deaths by cause from 1993. Further details can be found in the annual volumes Mortality statistics: Cause 1984, Series DH2 no. 11, and Mortality statistics: Cause 1993 (revised) and 1994, Series DH2 no. 21.

From 1 January 2001, under ICD-10, Rule 3 has again been changed - for details see the article in Health Statistics Quarterly13. This has resulted in a fall in the death rates from respiratory diseases, notably pneumonia, and consequently slight rises in the rates for other causes eg. strokes. For details of the major changes between ICD-9 and ICD-10, see the articles in Health Statistics Quarterly 08, 13 and 14.

\section{Age-standardised mortality rates}

Directly age-standardised rates make allowances for changes in the age structure of the population. The age-standardised rate for a particular condition is that which would have occurred if the observed age-specific rates for the condition had applied in a given standard population. Tables 2.2 and 6.3 use the European Standard Population. This is a hypothetical population standard which is the same for both males and females allowing standardised rates to be compared for each sex, and between males and females.

\section{Abortions}

Figures relate to numbers occurring in a period.

\section{Calculating quarterly rates}

Quarterly rates are calculated using seasonal adjustments which take into account the number of days in the month/year. The denominators used for calculating quarterly rates have been produced from mid-year population estimates and projections.

\section{Marriages and divorces}

Marriages are tabulated according to date of solemnisation. Divorces are tabulated according to date of decree absolute. In Scotland a small number of late divorces from previous years are added to the current year. The term 'divorces' includes decrees of nullity. The fact that a marriage or divorce has taken place in England, Wales, Scotland or Northern Ireland does not necessarily mean that either of the parties is resident there.

\section{Civil Partnerships}

The Civil Partnership Act 2004 came into force on 5 December 2005 in the UK, the first day couples could give notice of their intention to form a civil partnership. The first day that couples could normally form a partnership was 19 December 2005 in Northern Ireland, 20 December 2005 in Scotland and 21 December 2005 in England and Wales.

Civil partnerships are tabulated according to date of formation and area of occurrence. The fact that a civil partnership has taken place in England, Wales, Scotland or Northern Ireland does not necessarily mean either of the parties is resident there.

\section{Sources}

Figures for Scotland and Northern Ireland have been provided by the General Register Office for Scotland and the Northern Ireland Statistics and Research Agency respectively.

\section{Rounding}

All figures are rounded independently; constituent parts may not add to totals Generally numbers and rates per 1,000 population are rounded to one decimal place (for example 123.4); where appropriate, for small figures (below 10.0), two decimal places are given (for example 7.62). Figures which are provisional or estimated are given in less detail (for example 123 or 7.6 respectively) if their reliability does not justify giving the standard amount of detail. Where figures need to be treated with particular caution, an explanation is given as a footnote.

\section{Latest figures}

Figures for the latest quarters and years may be provisional and will be updated in future issues when later information becomes available. Where figures are not yet available, cells are left blank. 


\begin{tabular}{|c|c|c|c|c|c|c|c|c|c|c|c|c|c|}
\hline \multicolumn{2}{|c|}{ Table 1.1} & \multicolumn{12}{|c|}{ Population and vital rates: international } \\
\hline \multicolumn{11}{|c|}{ Selected countries } & \multicolumn{3}{|c|}{ Numbers (thousands)/Rates per thousand } \\
\hline Year & $\begin{array}{c}\text { United } \\
\text { Kingdom }\end{array}$ & Austria & Belgium & Bulgaria & Cyprus $^{1}$ & $\begin{array}{l}\text { Czech } \\
\text { Republic }\end{array}$ & Denmark & Estonia & Finland & France & Germany $^{2}$ & Greece $^{3}$ & Hungary \\
\hline \multicolumn{14}{|c|}{ Population (thousands) } \\
\hline 1971 & 55,928 & 7,501 & 9,673 & 8,540 & 610 & 9,810 & 4,963 & 1,369 & 4,612 & 51,251 & 78,313 & 8,831 & 10,370 \\
\hline 1976 & 56,216 & 7,566 & 9,818 & 8,760 & 498 & 10,094 & 5,073 & 1,435 & 4,726 & 52,909 & 78,337 & 9,167 & 10,590 \\
\hline 1981 & 56,357 & 7,569 & 9,859 & 8,891 & 515 & 10,293 & 5,121 & 1,482 & 4,800 & 54,182 & 78,408 & 9,729 & 10,712 \\
\hline 1986 & 56,684 & 7,588 & 9,862 & 8,958 & 545 & 10,340 & 5,120 & 1,534 & 4,918 & 55,547 & 77,720 & 9,967 & 10,631 \\
\hline 1991 & 57,439 & 7,813 & 9,979 & 8,982 & 587 & 10,309 & 5,154 & 1,566 & 5,014 & 57,055 & 79,984 & 10,247 & 10,346 \\
\hline 1996 & 58,164 & 7,959 & 10,137 & 8,363 & $661^{12}$ & 10,315 & 5,262 & 1,416 & 5,125 & 58,026 & 81,896 & 10,709 & 10,193 \\
\hline 2001 & 59,113 & 8,043 & 10,287 & 7,910 & $701^{12}$ & 10,224 & 5,359 & 1,364 & 5,188 & 59,322 & 82,340 & 10,950 & 10,188 \\
\hline 2002 & 59,323 & 8,084 & 10,333 & 7,869 & $710^{12}$ & 10,201 & 5,374 & 1,359 & 5,201 & 59,678 & 82,482 & 10,988 & 10,159 \\
\hline 2003 & 59,557 & 8,118 & 10,376 & 7,824 & $721^{12}$ & 10,202 & 5,387 & 1,354 & 5,213 & 60,028 & 82,520 & 11,024 & 10,130 \\
\hline 2004 & 59,846 & 8,175 & 10,421 & 7,781 & $737^{12}$ & 10,207 & 5,401 & 1,349 & 5,228 & 60,381 & 82,501 & 11,062 & 10,107 \\
\hline 2005 & 60,238 & 8,233 & 10,479 & 7,740 & $758^{12}$ & 10,234 & 5,416 & 1,346 & 5,246 & 60,996 & 82,464 & 11,104 & 10,087 \\
\hline 2006 & 60,587 & $8,280^{\mathrm{P}}$ & $10,511^{\mathrm{P}}$ & $7,680^{p}$ & $766^{12}$ & $10,280^{\mathrm{p}}$ & $5,427^{p}$ & 1,345 & 5,270 & $61,350^{\mathrm{P}}$ & $82,370^{\mathrm{P}}$ & $11,150^{\mathrm{p}}$ & $10,077^{\mathrm{p}}$ \\
\hline 2007 & 60,975 & .. & .. & .. & .. & .. & .. & $1,340^{\mathrm{p}}$ & $5,290^{\mathrm{p}}$ & .. & $82,260^{p}$ & .. & .. \\
\hline \multicolumn{14}{|c|}{ Population changes (per 1,000 per annum) } \\
\hline $1971-76$ & 1.0 & 1.7 & 3.0 & 5.2 & -36.7 & 5.8 & 4.4 & 9.6 & 4.9 & 6.5 & 0.1 & 7.6 & 4.2 \\
\hline 1976-81 & 0.5 & 0.1 & 0.8 & 3.0 & 6.8 & 3.9 & 1.9 & 6.6 & 3.1 & 4.8 & 0.2 & 12.3 & 2.3 \\
\hline $1981-86$ & 1.2 & 0.5 & 0.1 & 1.5 & 11.7 & 0.9 & 0.0 & 7.0 & 4.9 & 5.0 & -1.8 & 4.9 & -1.5 \\
\hline 1986-91 & 2.7 & 5.9 & 2.4 & 0.5 & 15.4 & -0.6 & 1.3 & 4.2 & 3.9 & 5.4 & 5.8 & 5.6 & -5.4 \\
\hline 1991-96 & 2.5 & 3.7 & 3.6 & -13.8 & 25.2 & 0.1 & 4.2 & -12.4 & 3.8 & 3.4 & 4.8 & 9.0 & -3.0 \\
\hline 1996-01 & 3.3 & 2.1 & 2.6 & -10.8 & 12.1 & -1.8 & 3.7 & -7.3 & 2.5 & 4.5 & 1.1 & 4.5 & -0.1 \\
\hline 2001-02 & 3.5 & 5.1 & 4.5 & -5.2 & 12.8 & -2.2 & 2.8 & -3.7 & 2.5 & 6.0 & 1.7 & 4.4 & -2.8 \\
\hline $2002-03$ & 3.9 & 4.2 & 4.2 & -5.7 & 15.5 & 0.1 & 2.4 & -3.7 & 2.3 & 5.9 & 0.5 & 2.4 & -2.9 \\
\hline 2003-04 & 4.8 & 7.0 & 4.3 & -5.5 & 22.2 & 0.5 & 2.6 & -3.7 & 2.9 & 5.9 & -0.2 & 3.4 & -2.3 \\
\hline 2004-05 & 6.6 & 7.1 & 5.6 & -5.3 & 28.5 & 2.6 & 2.8 & -2.2 & 3.4 & 10.2 & -0.4 & 3.8 & -2.0 \\
\hline $2005-06$ & 5.8 & $5.7^{p}$ & $3.1^{\mathrm{P}}$ & $-7.8^{p}$ & 10.6 & $4.5^{\mathrm{P}}$ & $2.0^{p}$ & -0.7 & 4.6 & $5.8^{p}$ & $-1.1^{p}$ & $4.1^{\mathrm{p}}$ & $-1.0^{p}$ \\
\hline $2006-07$ & 6.4 & .. & .. &.. & .. & .. &.. & $-3.7^{p}$ & $3.8^{\mathrm{p}}$ & .. & $-1.3^{p}$ & .. &.. \\
\hline \multicolumn{14}{|c|}{ Live birth rate (per 1,000 population per annum) } \\
\hline $1971-75$ & 14.1 & 13.3 & 13.4 & 13.2 & 17.7 & 17.8 & 14.6 & 15.4 & 13.1 & 16.0 & 10.5 & 15.8 & 16.1 \\
\hline $1976-80$ & 12.5 & 11.5 & 12.5 & 15.1 & 19.0 & 17.1 & 12.0 & 15.0 & 13.6 & 14.1 & 10.5 & 15.6 & 15.8 \\
\hline $1981-85$ & 12.9 & 12.0 & 12.0 & 13.7 & 20.2 & 13.5 & 10.2 & 15.6 & 13.4 & 14.2 & 10.7 & 13.3 & 12.3 \\
\hline $1986-90$ & 13.7 & 11.6 & 12.1 & 12.7 & 18.8 & 12.7 & 11.5 & 15.5 & 12.7 & 13.8 & 9.8 & 10.6 & 11.8 \\
\hline $1991-95$ & 13.2 & 11.8 & 12.0 & 9.8 & 16.9 & 11.1 & 13.1 & 10.7 & 12.9 & 12.7 & 10.9 & 9.9 & 11.7 \\
\hline $1996-00$ & 12.0 & 10.2 & 11.2 & 8.3 & 13.2 & 8.8 & 12.6 & 8.9 & 11.3 & 12.7 & 9.6 & 10.2 & 9.8 \\
\hline 2001 & 11.3 & 9.4 & 11.1 & 8.6 & 11.6 & 8.9 & 12.2 & 9.3 & 10.8 & 13.0 & 8.9 & 9.3 & 9.5 \\
\hline 2002 & 11.3 & 9.7 & 10.8 & 8.5 & 11.1 & 9.6 & 11.9 & 9.6 & 10.7 & 12.7 & 8.7 & 9.5 & 9.5 \\
\hline 2003 & 11.7 & 9.5 & 10.9 & 8.6 & 11.2 & 9.2 & 12.0 & 9.6 & 10.9 & 12.7 & 8.6 & 9.5 & 9.3 \\
\hline 2004 & 12.0 & 9.7 & 11.1 & 9.0 & 11.3 & 9.6 & 11.9 & 10.4 & 11.0 & 12.7 & 8.6 & 9.6 & 9.4 \\
\hline 2005 & 12.0 & 9.5 & 11.2 & 9.2 & 10.9 & 10.0 & 11.9 & 10.7 & 11.0 & 12.7 & 8.3 & 9.7 & 9.7 \\
\hline 2006 & 12.4 & 9.3 & 11.5 & 9.6 & 11.3 & 10.3 & 12.0 & 11.1 & 11.2 & 13.0 & 8.2 & 10.0 & 9.9 \\
\hline 2007 & 12.7 & 9.1 & 11.4 & 9.8 & 10.8 & 11.1 & 11.7 & 11.7 & 11.1 & 12.8 & 8.3 & 9.8 & 9.7 \\
\hline \multicolumn{14}{|c|}{ Death rate (per $1,000 p$} \\
\hline $1971-75$ & 11.8 & 12.6 & 12.1 & 9.8 & 9.9 & 12.4 & 10.1 & 11.1 & 9.5 & 10.7 & 12.3 & 8.6 & 11.9 \\
\hline $1976-80$ & 11.9 & 12.3 & 11.6 & 12.9 & 10.4 & 12.5 & 10.5 & 12.1 & 9.3 & 10.2 & 12.2 & 8.8 & 12.9 \\
\hline $1981-85$ & 11.7 & 12.0 & 11.4 & 11.3 & 10.0 & 12.8 & 11.1 & 12.3 & 9.3 & 10.1 & 12.0 & 9.0 & 13.7 \\
\hline $1986-90$ & 11.4 & 11.1 & 10.8 & 11.9 & 10.2 & 12.4 & 11.5 & 11.9 & 9.8 & 9.5 & 11.6 & 9.3 & 13.5 \\
\hline $1991-95$ & 11.1 & 10.4 & 10.4 & 12.9 & 9.0 & 11.6 & 11.9 & 13.9 & 9.8 & 9.1 & 10.8 & 9.5 & 14.3 \\
\hline $1996-00$ & 10.6 & 9.7 & 10.3 & 14.0 & 7.7 & 10.8 & 11.2 & 13.1 & 9.6 & 9.2 & 10.4 & 9.7 & 13.9 \\
\hline 2001 & 10.2 & 9.3 & 10.1 & 14.2 & 6.9 & 10.5 & 10.9 & 13.6 & 9.4 & 8.9 & 10.1 & 9.4 & 13.0 \\
\hline 2002 & 10.2 & 9.4 & 10.2 & 14.3 & 7.3 & 10.6 & 10.9 & 13.5 & 9.5 & 9.2 & 10.2 & 9.5 & 13.1 \\
\hline 2003 & 10.3 & 9.5 & 10.4 & 14.3 & 7.2 & 10.9 & 10.7 & 13.4 & 9.4 & 9.2 & 10.3 & 9.6 & 13.4 \\
\hline 2004 & 9.7 & 9.1 & 9.8 & 14.2 & 7.1 & 10.5 & 10.3 & 13.2 & 9.1 & 8.4 & 10.0 & 9.5 & 13.1 \\
\hline 2005 & 9.7 & 9.1 & 9.8 & 14.6 & 7.2 & 10.6 & 10.2 & 12.9 & 9.1 & 8.6 & 10.1 & 9.5 & 13.5 \\
\hline 2006 & 9.4 & 8.9 & .. & 14.8 & 6.7 & 10.2 & 10.2 & 12.9 & 9.1 & .. & 9.9 & 9.5 & 13.1 \\
\hline 2007 & 9.4 & 8.9 & .. & .. & .. & .. & 10.2 & .. & 9.2 & .. & 10.0 & .. & 13.0 \\
\hline
\end{tabular}

Note: as given in the United Nations Monthly Bulletin of Statistics (June 2008), the United Nation Demographic Yearbook (May 2008), and the Eurostat website (June 2008).

1 Republic of Cyprus - Greek Cypriot controlled area only

2 Including former GDR throughout.

3 Greece - mid-year population excludes armed forces stationed outside the country but includes alien forces stationed in the area.

4 Malta - including work and resident permit holders and foreigners residing in Malta.

5 Poland - excluding civilian aliens within the country but including civilian nationals temporarily outside the country. Average year data for 2000 and 2001 contain revised data according to the final results of the population census 2002.

6 Portugal - including the Azores and Madeira islands.

7 Spain - including the Balearic and Canary Islands.

8 For 1971 the European Union consisted of the 6 original member countries. This has since been expanded to include: 9 countries (1976-EU15); 10 countries (2004-EU25); 2 countries (2007-EU27). In this table, all totals include the EU27.
9 Including the Indian held part of Jammu and Kashmir, the final status of which has not yet been determined.

10 Japan - excluding diplomatic personnel outside the country and foreign military and civilian personnel and their dependants stationed in the area. Rates are based on births to or deaths of Japanese nationals only.

11 USA - excluding armed forces overseas and civilian citizens absent from the country for extended periods.

12 Indicates population estimates of uncertain reliability.

13 Data refer to 15 April.

14 Figures were updated taking into account the results of the 2002 All Russian Population Census.

15 Mid-year estimates have been adjusted for under-enumeration

16 For statistical purposes the data for China do not include those for the Hong Kong SAR, Macao SAR and Taiwan province of China. Data for the period 1996 to 2000 have been adjusted on the basis of the Population Census of 2000. Data from 2001 to 2004 have been estimated on the basis of the annual national sample surveys of Population Changes. Estimate of uncertain reliability. Death rates for 1999-2003 and birth rates for 2000-2003 were obtained by the Sample Survey of Population Change 2003 in China.

17 Rate is for $1990-1995$

p provisional. 


\begin{tabular}{|c|c|c|c|c|c|c|c|c|c|c|c|c|c|c|}
\hline \multicolumn{2}{|c|}{$\begin{array}{l}\text { Table } 1.1 \\
\text { continued }\end{array}$} & \multicolumn{13}{|c|}{ Population and vital rates: international } \\
\hline \multicolumn{11}{|c|}{ Selected countries } & \multicolumn{4}{|c|}{ Numbers (thousands)/Rates per thousand } \\
\hline Year & $\begin{array}{c}\text { Irish } \\
\text { Republic }\end{array}$ & Italy & Latvia & Lithuania & $\begin{array}{l}\text { Luxem- } \\
\text { bourg }\end{array}$ & Malta $^{4}$ & $\begin{array}{l}\text { Nether- } \\
\text { lands }\end{array}$ & Poland $^{5}$ & Portugal $^{6}$ & Romania & Slovakia & Slovenia & Spain $^{7}$ & Sweden \\
\hline \multicolumn{15}{|c|}{ Population (thousands) } \\
\hline 1971 & 2,992 & 54,073 & 2,366 & 3,160 & 342 & 330 & 13,194 & 32,800 & 8,644 & 20,470 & 4,540 & 1,732 & 34,216 & 8,098 \\
\hline 1976 & 3,238 & 55,718 & 2,465 & 3,315 & 361 & 330 & 13,774 & 34,360 & 9,356 & 21,450 & 4,764 & 1,809 & 36,118 & 8,222 \\
\hline 1981 & 3,443 & 56,502 & 2,515 & 3,422 & 365 & 322 & 14,247 & 35,902 & 9,851 & 22,353 & 4,996 & 1,910 & 37,741 & 8,320 \\
\hline 1986 & 3,543 & 56,596 & 2,588 & 3,560 & 368 & 344 & 14,572 & 37,456 & 10,011 & 22,823 & 5,179 & 1,975 & 38,536 & 8,370 \\
\hline 1991 & 3,526 & 56,751 & 2,662 & 3,742 & 387 & 358 & 15,070 & 38,245 & 9,871 & 23,185 & 5,283 & 2,002 & 38,920 & 8,617 \\
\hline 1996 & $3,626^{13}$ & 56,860 & 2,457 & 3,602 & 414 & 380 & 15,530 & 38,618 & 10,058 & 22,608 & 5,374 & 1,991 & 39,479 & 8,841 \\
\hline 2001 & $3,839^{13}$ & 56,978 & 2,355 & 3,481 & 442 & 393 & 16,046 & 38,251 & 10,293 & 22,408 & 5,380 & 1,992 & 40,721 & 8,896 \\
\hline 2002 & $3,917^{13}$ & 57,157 & 2,339 & 3,469 & 446 & 396 & 16,149 & 38,232 & 10,368 & 21,795 & 5,379 & 1,996 & 41,314 & 8,925 \\
\hline 2003 & $3,996^{13}$ & 57,605 & 2,325 & 3,454 & 450 & 399 & 16,225 & 38,195 & 10,441 & 21,734 & 5,379 & 1,997 & 42,005 & 8,958 \\
\hline 2004 & $4,044^{13}$ & 58,175 & 2,313 & 3,436 & 453 & 401 & 16,282 & 38,180 & 10,502 & 21,673 & 5,382 & 1,997 & 42,692 & 8,994 \\
\hline 2005 & $4,131^{13}$ & 58,607 & 2,301 & 3,414 & 457 & 404 & 16,320 & 38,161 & 10,549 & 21,624 & 5,387 & 2,001 & 43,398 & 9,030 \\
\hline 2006 & $4,230^{13}$ & 58,940 & 2,295 & $3,390^{\mathrm{p}}$ & $470^{p}$ & $410^{p}$ & $16,350^{p}$ & 38,130 & 10,580 & $21,580^{p}$ & $5,400^{p}$ & 2,010 & 44,100 & $9,090^{p}$ \\
\hline 2007 & $4,340^{\mathrm{P}}$ & $59,420^{p}$ & $2,270^{\mathrm{P}}$ & $3,370^{p}$ & & $410^{p}$ & $16,370^{p}$ & .. & .. & $21,540^{p}$ & $5,390^{p}$ & $2,020^{\mathrm{p}}$ & $44,850^{p}$ & $9,140^{p}$ \\
\hline \multicolumn{15}{|c|}{ Population changes (per 1,000 per annum) } \\
\hline $1971-76$ & 16.4 & 6.1 & 8.4 & 9.8 & 10.7 & 0.0 & 8.8 & 9.5 & 16.5 & 9.6 & 9.9 & 8.9 & 11.1 & 3.1 \\
\hline $1976-81$ & 12.7 & 2.8 & 4.1 & 6.5 & 2.5 & -4.8 & 6.9 & 9.0 & 10.6 & 8.4 & 9.7 & 11.2 & 9.0 & 2.4 \\
\hline $1981-86$ & 5.8 & 0.3 & 5.8 & 8.1 & 1.8 & 13.7 & 4.6 & 8.7 & 3.2 & 4.2 & 7.3 & 6.8 & 4.2 & 1.2 \\
\hline $1986-91$ & -1.0 & 0.5 & 5.7 & 10.2 & 10.2 & 8.1 & 6.8 & 4.2 & -2.8 & 3.2 & 4.0 & 2.7 & 2.0 & 5.9 \\
\hline 1991-96 & 4.3 & 0.4 & -12.8 & -1.7 & 13.9 & 8.4 & 6.1 & 2.0 & 3.8 & -5.0 & 3.4 & -1.1 & 2.9 & 5.1 \\
\hline $1996-01$ & 11.7 & 0.4 & -8.3 & -6.7 & 13.5 & 6.8 & 6.6 & -1.9 & 4.7 & -1.8 & 0.2 & 0.1 & 6.3 & 1.2 \\
\hline 2001-02 & 20.3 & 3.1 & -6.8 & -3.4 & 9.0 & 7.6 & 6.4 & -0.5 & 7.3 & -27.4 & -0.2 & 2.0 & 14.6 & 3.3 \\
\hline $2002-03$ & 20.2 & 7.8 & -6.0 & -4.3 & 9.0 & 7.6 & 4.7 & -1.0 & 7.0 & -2.8 & 0.0 & 0.5 & 16.7 & 3.7 \\
\hline 2003-04 & 12.0 & 9.9 & -5.2 & -5.2 & 6.7 & 5.0 & 3.5 & -0.4 & 5.8 & -2.8 & 0.6 & 0.0 & 16.4 & 4.0 \\
\hline 2004-05 & 21.5 & 7.4 & -5.2 & -6.4 & 8.8 & 7.5 & 2.3 & -0.5 & 4.5 & -2.3 & 0.9 & 2.0 & 16.5 & 4.0 \\
\hline 2005-06 & 24.0 & 5.7 & -2.6 & $-7.0^{\mathrm{p}}$ & $28.4^{\mathrm{P}}$ & $14.9^{\mathrm{p}}$ & $1.8^{p}$ & -0.8 & 2.9 & $-2.0^{p}$ & $2.4^{p}$ & 4.5 & 16.2 & $6.6^{p}$ \\
\hline 2006-07 & $26.0^{\mathrm{P}}$ & $8.1^{\mathrm{P}}$ & $-10.9^{p}$ & $-5.9^{p}$ & .. & $0.0^{p}$ & $1.2^{p}$ & .. & .. & $-1.9^{P}$ & $-1.9^{P}$ & $5.0^{\mathrm{p}}$ & $17.0^{p}$ & $5.5^{\mathrm{p}}$ \\
\hline \multicolumn{15}{|c|}{ Live birth rate (per 1,000 population per annum) } \\
\hline 1971-75 & 22.2 & 16.0 & 14.4 & 16.4 & 11.6 & 17.5 & 14.9 & 17.9 & 20.3 & 19.3 & 19.7 & 16.4 & 19.2 & 13.5 \\
\hline $1976-80$ & 21.3 & 12.6 & 13.9 & 15.4 & 11.2 & 17.0 & 12.6 & 19.3 & 17.9 & 18.9 & 20.3 & 16.3 & 17.1 & 11.6 \\
\hline $1981-85$ & 19.2 & 10.6 & 15.2 & 16.0 & 11.6 & 15.3 & 12.2 & 19.0 & 14.5 & 15.6 & 18.0 & 14.2 & 12.8 & 11.3 \\
\hline $1986-90$ & 15.8 & 9.8 & 15.3 & 15.8 & 12.2 & 16.0 & 12.8 & 15.5 & 11.9 & 15.8 & 15.8 & 12.3 & 10.8 & 13.2 \\
\hline $1991-95$ & 14.0 & 9.6 & 10.8 & 13.1 & 13.3 & 14.0 & 12.8 & 12.9 & 11.4 & 11.1 & 13.3 & 10.0 & 9.8 & 13.3 \\
\hline $1996-00$ & 14.2 & 9.2 & 8.0 & 10.4 & 13.1 & 12.0 & 12.6 & 10.4 & 11.3 & 10.4 & 10.7 & 9.1 & 9.5 & 10.2 \\
\hline 2001 & 15.1 & 9.2 & 8.3 & 9.1 & 12.4 & 10.0 & 12.6 & 9.6 & 11.0 & 9.8 & 9.5 & 8.8 & 10.0 & 10.3 \\
\hline 2002 & 15.5 & 9.4 & 8.6 & 8.7 & 12.0 & 9.6 & 12.5 & 9.3 & 11.0 & 9.7 & 9.5 & 8.8 & 10.2 & 10.7 \\
\hline 2003 & 15.4 & 9.4 & 9.0 & 8.9 & 11.8 & 10.1 & 12.3 & 9.2 & 10.8 & 9.8 & 9.6 & 8.7 & 10.5 & 11.1 \\
\hline 2004 & 15.3 & 9.7 & 8.8 & 8.9 & 11.8 & 9.7 & 11.9 & 9.3 & 10.4 & 10.0 & 10.0 & 9.0 & 10.6 & 11.2 \\
\hline 2005 & 14.8 & 9.5 & 9.4 & 9.0 & 11.8 & 9.6 & 11.5 & 9.6 & 10.4 & 10.2 & 9.3 & 9.1 & 10.7 & 11.2 \\
\hline 2006 & 15.2 & 9.5 & 9.7 & 9.2 & 11.7 & 9.6 & 11.3 & 9.8 & 10.0 & 10.1 & 10.0 & 9.4 & 10.9 & 11.7 \\
\hline 2007 & 16.2 & 9.5 & 10.1 & 9.5 & 11.4 & 9.5 & 11.0 & 10.2 & 10.0 & 9.9 & 10.1 & 9.7 & 10.8 & 11.7 \\
\hline \multicolumn{15}{|c|}{ Death rate (per 1,000 population per annum) } \\
\hline $1971-75$ & 11.0 & 9.8 & 11.6 & 9.0 & 12.2 & 9.0 & 8.3 & 8.4 & 11.0 & 9.4 & 9.4 & 10.0 & 8.5 & 10.5 \\
\hline $1976-80$ & 10.2 & 9.7 & 12.6 & 10.1 & 11.5 & 9.0 & 8.1 & 9.2 & 10.1 & 9.8 & 9.8 & 9.8 & 8.0 & 10.9 \\
\hline 1981-85 & 9.4 & 9.5 & 12.8 & 10.6 & 11.2 & 8.2 & 8.3 & 9.6 & 9.6 & 10.3 & 10.1 & 10.3 & 7.7 & 11.0 \\
\hline $1986-90$ & 9.1 & 9.4 & 12.4 & 10.3 & 10.5 & 7.4 & 8.5 & 10.0 & 9.6 & 10.8 & 10.1 & 9.6 & 8.2 & 11.1 \\
\hline $1991-95$ & 8.8 & 9.7 & 14.8 & 12.0 & 9.8 & 7.6 & 8.8 & 10.2 & 10.4 & 11.5 & 9.9 & 9.7 & 8.7 & 10.9 \\
\hline $1996-00$ & 8.5 & 9.8 & 13.9 & 11.5 & 9.0 & 7.7 & 8.8 & 9.8 & 10.5 & 12.0 & 9.7 & 9.5 & 9.1 & 10.6 \\
\hline 2001 & 7.9 & 9.6 & 14.0 & 11.6 & 8.4 & 7.6 & 8.7 & 9.5 & 10.2 & 11.6 & 9.7 & 9.3 & 8.9 & 10.5 \\
\hline 2002 & 7.5 & 9.8 & 13.9 & 11.8 & 8.4 & 7.8 & 8.8 & 9.4 & 10.2 & 12.4 & 9.6 & 9.4 & 8.9 & 10.6 \\
\hline 2003 & 7.2 & 10.2 & 13.9 & 11.9 & 9.0 & 7.7 & 8.7 & 9.6 & 10.4 & 12.3 & 9.7 & 9.7 & 9.2 & 10.4 \\
\hline 2004 & 7.0 & 9.4 & 13.9 & 12.0 & 7.6 & 7.2 & 8.4 & 9.5 & 9.7 & 11.9 & 9.6 & 9.3 & 8.7 & 10.1 \\
\hline 2005 & 6.6 & 9.7 & 14.2 & 12.8 & 8.0 & 7.8 & 8.4 & 9.7 & 10.2 & 12.1 & 9.9 & 9.4 & 8.9 & 10.2 \\
\hline 2006 & 6.5 & 9.5 & 14.5 & 13.2 & 8.0 & .. & 8.3 & 9.7 & 9.7 & 11.9 & 9.9 & 9.1 & 8.4 & 10.0 \\
\hline 2007 & .. & & 14.5 & 13.5 & .. & .. & .. &.. & .. & 11.7 & 9.9 & .. & .. & 10.0 \\
\hline
\end{tabular}

See notes on first page of table. 


\section{Table 1.1 continued}

Population and vital rates: international

Selected countries

\begin{tabular}{|c|c|c|c|c|c|c|c|c|c|c|}
\hline \multicolumn{8}{|c|}{ Selected countries } & \multicolumn{3}{|c|}{ Numbers (thousands)/Rates per thousand } \\
\hline Year & $\mathrm{EU}^{8}$ & $\begin{array}{l}\text { Russian } \\
\text { Federation }\end{array}$ & Australia & Canada & $\begin{array}{c}\text { New } \\
\text { Zealand }\end{array}$ & China & India ${ }^{9}$ & $J^{\prime a p a n}{ }^{10}$ & USA $^{11}$ & Year \\
\hline \multicolumn{11}{|c|}{ Population (thousands) } \\
\hline 1971 & 438,728 & 130,934 & 13,067 & 22,026 & 2,899 & $852,290^{16}$ & 551,311 & 105,145 & 207,661 & 1971 \\
\hline 1976 & 450,468 & 135,027 & 14,033 & 23,517 & 3,163 & $937,170^{16}$ & 617,248 & 113,094 & 218,035 & 1976 \\
\hline 1981 & 459,807 & 139,225 & 14,923 & 24,900 & 3,195 & $1,008,460^{16}$ & 675,185 & 117,902 & 229,958 & 1981 \\
\hline 1986 & 465,336 & 144,154 & 16,018 & 26,204 & 3,317 & $1,086,733^{16}$ & 767,199 & 121,672 & 240,680 & 1986 \\
\hline 1991 & 473,094 & 148,245 & 17,284 & 28,031 & 3,477 & $1,170,100^{16}$ & 851,897 & 123,964 & 252,639 & 1991 \\
\hline 1996 & 478,084 & $148,160^{14}$ & $18,311^{15}$ & $29,611^{15}$ & 3,732 & $1,217,550^{16}$ & $942,157^{12}$ & 125,757 & 269,394 & 1996 \\
\hline 2001 & 482,464 & $145,976^{14}$ & $19,413^{15}$ & $31,021^{15}$ & 3,880 & $1,271,850^{16}$ & $1,035,066^{12}$ & 127,130 & 285,108 & 2001 \\
\hline 2002 & 483,643 & $145,306^{14}$ & $19,641^{15}$ & $31,373^{15}$ & 3,939 & $1,280,400^{16}$ & $1,050,640^{12}$ & 127,400 & 287,985 & 2002 \\
\hline 2003 & 485,617 & $144,566^{14}$ & $19,873^{15}$ & $31,669^{15}$ & 4,009 & $1,288,400^{16}$ & $1,068,214^{12}$ & 127,650 & 290,850 & 2003 \\
\hline 2004 & 487,720 & $143,821^{14}$ & $20,111^{15}$ & $31,974^{15}$ & 4,061 & $1,296,075^{16}$ & $1,085,600^{12}$ & 127,670 & 293,623 & 2004 \\
\hline 2005 & 490,125 & $143,150^{14}$ & $20,409^{15}$ & $32,312^{15}$ & 4,099 & $1,303,720^{16}$ & $1,101,000^{12}$ & 127,773 & 296,410 & 2005 \\
\hline 2006 & $492,068^{P}$ & $142,490^{14}$ & $20,700^{15}$ & $32,650^{15}$ & 4,180 & $1,311,020^{16}$ & $1,117,730^{12}$ & 127,760 & 299,400 & 2006 \\
\hline 2007 & .. & .. & $21,020^{15, P}$ & $32,980^{15, P}$ & $4,230^{P}$ & .. & $1,134,000^{12}$ & 127,770 & .. & 2007 \\
\hline
\end{tabular}

Population changes (per 1,000 per annum)

$\begin{array}{ll}1971-76 & 5.4 \\ 1976-81 & 4.1 \\ 1981-86 & 2.4 \\ 1986-91 & 3.3 \\ 1991-96 & 2.1 \\ 1996-01 & 1.8 \\ & \\ 2001-02 & 2.4 \\ 2002-03 & 4.1 \\ 2003-04 & 4.3 \\ 2004-05 & 4.9 \\ 2005-06 & 3.8^{\text {p }} \\ 2006-07 & \end{array}$

$\begin{array}{rrr}6.3 & 14.8 & 13.5 \\ 6.2 & 12.7 & 11.8 \\ 7.1 & 14.7 & 10.5 \\ 5.7 & 15.8 & 13 . \\ -1.7 & 11.9 & 11.3 \\ -2.9 & 12.0 & 9.5 \\ -4.6 & 11.7 & 11.3 \\ -5.1 & 11.8 & 9.4 \\ -5.2 & 12.0 & 9.6 \\ -4.7 & 14.8 & 10.6 \\ -4.6 & 14.3 & 10.5 \\ . . & 15.5^{P} & 10.1^{p}\end{array}$

$\begin{array}{rr}13.5 & 18 . \\ 11.8 & 2.0 \\ 10.5 & 7.6 \\ 13.9 & 9 . \\ 11.3 & 14.7 \\ 9.5 & 7.9 \\ & \\ 11.3 & 15 . \\ 9.4 & 17.8 \\ 9.6 & 13.0 \\ 10.6 & 9.4 \\ 10.5 & 19.8 \\ 10.1^{p} & 12 .\end{array}$

$\begin{array}{rrr}18.2 & 19.9 & 23.9 \\ 2.0 & 15.2 & 18.8 \\ 7.6 & 15.5 & 27.3 \\ 9.6 & 15.3 & 22.1 \\ 14.7 & 10.3 & 21 . \\ 7.9 & 8.9 & 19.7 \\ & & \\ 15.2 & 6.7 & 15.0 \\ 17.8 & 6.2 & 16.7 \\ 13.0 & 6.0 & 16.3 \\ 9.4 & 5.9 & 14.2 \\ 19.8 & 5.6 & 15.2 \\ 12.0^{\text {p }} & . . & 14.6 \\ & & \end{array}$

$\begin{array}{rr}23.9 & 15 . \\ 18.8 & 8.5 \\ 27.3 & 6.4 \\ 22.1 & 3.8 \\ 21.1 & 2.9 \\ 19.7 & 2.2 \\ & \\ 15.0 & 2.1 \\ 16.7 & 2.0 \\ 16.3 & 0.2 \\ 14.2 & 0.8 \\ 15.2 & -0.1 \\ 14.6 & 0.1\end{array}$

$\begin{array}{rrr}15.1 & 10.0 & 1971-76 \\ 8.5 & 10.9 & 1976-81 \\ 6.4 & 9.3 & 1981-86 \\ 3.8 & 9.9 & 1986-91 \\ 2.9 & 12.1 & 1991-96 \\ 2.2 & 11.7 & 1996-01 \\ & & \\ 2.1 & 10.1 & 2001-02 \\ 2.0 & 9.9 & 2002-03 \\ 0.2 & 9.5 & 2003-04 \\ 0.8 & 9.5 & 2004-05 \\ -0.1 & 10.1 & 2005-06 \\ 0.1 & . . & 2006-07\end{array}$

Live birth rate (per 1,000 population per annum)

1971-75

$1976-80$

1981-85

1986-90

1991-95

1996-00

$\begin{array}{rr}. . & . . \\ . . & . . \\ . . & . . \\ . . & . . \\ 11.4 & 10.2 \\ 10.6 & 8.6 \\ & \\ 10.1 & 9.0 \\ 10.3 & 9.6 \\ 10.3 & 10.2 \\ 10.4 & 10.5 \\ 10.4 & 10.2 \\ 10.6 & 10.4 \\ 10.6 & 10.9\end{array}$

18.8

15.9

15.5

15.1

15.6

$15.1 \quad 14.8$

2001

10.2
8.6

14.7
13.4

13.6
11.4

20.4

27.2

35.6

$\begin{array}{cr}18.6 & 33.4 \\ 19.2 & . . \\ . . & . . \\ 18.5^{17} & . . \\ . . & . . \\ 13.4^{16} & 25.4 \\ 12.9^{16} & 25.0 \\ 12.4^{16} & 24.8 \\ 12.3^{16} & 24.1 \\ 12.4^{16} & 23.8\end{array}$

18.6
14.9
12.6
10.6
9.7
9.5
9.2
9.1
8.8
8.7
8.3
8.6

\begin{tabular}{rr}
15.3 & $1971-75$ \\
15.2 & $1976-80$ \\
15.7 & $1981-85$ \\
16.0 & $1986-90$ \\
13.1 & $1991-95$ \\
14.3 & $1996-00$ \\
14.1 & \\
14.0 & 2001 \\
14.1 & 2002 \\
14.0 & 2003 \\
14.0 & 2004 \\
.. & 2005 \\
.. & 2006 \\
\hline & 2007
\end{tabular}

Death rate (per 1,000 population per annum)

\begin{tabular}{|c|c|c|c|c|c|c|c|c|c|c|}
\hline $1971-75$ & .. & .. & 8.2 & 7.4 & 8.4 & 7.3 & 15.5 & 6.4 & 9.1 & $1971-75$ \\
\hline $1976-80$ & .. &.. & 7.6 & 7.2 & 8.2 & 6.6 & 13.8 & 6.1 & 8.7 & 1976-80 \\
\hline $1981-85$ & .. &.. & 7.3 & 7.0 & 8.1 & 6.7 & .. & 6.1 & 8.6 & 1981-85 \\
\hline $1986-90$ & .. &.. & 7.2 & 7.3 & 8.2 & .. & .. & 6.4 & 8.7 & 1986-90 \\
\hline 1991-95 & 10.4 & 13.7 & 7.0 & 7.8 & 7.8 & .. & .. & 7.0 & 8.7 & 1991-95 \\
\hline 1996-00 & 10.2 & 14.3 & 6.9 & 7.2 & 7.2 & .. & .. & 7.4 & 8.5 & 1996-00 \\
\hline 2001 & 9.9 & 15.4 & 6.6 & 7.1 & 7.2 & $6.4^{16}$ & 8.4 & 7.6 & 8.5 & 2001 \\
\hline 2002 & 9.9 & 16.1 & 6.8 & 7.1 & 7.1 & $6.4^{16}$ & 8.1 & 7.7 & 8.5 & 2002 \\
\hline 2003 & 10.1 & 16.4 & 6.7 & 7.1 & 7.0 & $6.4^{16}$ & 8.0 & 8.0 & 8.4 & 2003 \\
\hline 2004 & 9.7 & 16.0 & 6.6 & 7.1 & 7.0 & $6.4^{16}$ & 7.5 & 8.1 & 8.2 & 2004 \\
\hline 2005 & 9.8 & 15.2 & 6.4 & 7.2 & 6.6 & $6.5^{16}$ & 7.6 & 8.5 & .. & 2005 \\
\hline 2006 & .. & 15.0 & 6.5 & .. & 6.7 & .. & .. & 8.5 & .. & 2006 \\
\hline 2007 & .. & .. & .. & .. & 6.8 & .. & .. & .. & .. & 2007 \\
\hline
\end{tabular}

See notes on first page of table. 


\section{Table 1.2}

Population: national

\begin{tabular}{|c|c|c|c|c|c|c|c|}
\hline \multicolumn{5}{|c|}{ Constituent countries of the United Kingdom } & \multicolumn{3}{|c|}{ Numbers (thousands) and percentage age distribution } \\
\hline Mid-year & $\begin{array}{c}\text { United } \\
\text { Kingdom }\end{array}$ & $\begin{array}{l}\text { Great } \\
\text { Britain }\end{array}$ & $\begin{array}{l}\text { England } \\
\text { and Wales }\end{array}$ & England & Wales & Scotland & $\begin{array}{l}\text { Northern } \\
\text { Ireland }\end{array}$ \\
\hline \multicolumn{8}{|l|}{ Estimates } \\
\hline 1971 & 55,928 & 54,388 & 49,152 & 46,412 & 2,740 & 5,236 & 1,540 \\
\hline 1976 & 56,216 & 54,693 & 49,459 & 46,660 & 2,799 & 5,233 & 1,524 \\
\hline 1981 & 56,357 & 54,815 & 49,634 & 46,821 & 2,813 & 5,180 & 1,543 \\
\hline 1986 & 56,684 & 55,110 & 49,999 & 47,188 & 2,811 & 5,112 & 1,574 \\
\hline 1991 & 57,439 & 55,831 & 50,748 & 47,875 & 2,873 & 5,083 & 1,607 \\
\hline 1993 & 57,714 & 56,078 & 50,986 & 48,102 & 2,884 & 5,092 & 1,636 \\
\hline 1994 & 57,862 & 56,218 & 51,116 & 48,229 & 2,887 & 5,102 & 1,644 \\
\hline 1995 & 58,025 & 56,376 & 51,272 & 48,383 & 2,889 & 5,104 & 1,649 \\
\hline 1996 & 58,164 & 56,503 & 51,410 & 48,519 & 2,891 & 5,092 & 1,662 \\
\hline 1997 & 58,314 & 56,643 & 51,560 & 48,665 & 2,895 & 5,083 & 1,671 \\
\hline 1998 & 58,475 & 56,797 & 51,720 & 48,821 & 2,900 & 5,077 & 1,678 \\
\hline 1999 & 58,684 & 57,005 & 51,933 & 49,033 & 2,901 & 5,072 & 1,679 \\
\hline 2000 & 58,886 & 57,203 & 52,140 & 49,233 & 2,907 & 5,063 & 1,683 \\
\hline 2001 & 59,113 & 57,424 & 52,360 & 49,450 & 2,910 & 5,064 & 1,689 \\
\hline 2002 & 59,323 & 57,627 & 52,572 & 49,652 & 2,920 & 5,055 & 1,697 \\
\hline 2003 & 59,557 & 57,855 & 52,797 & 49,866 & 2,931 & 5,057 & 1,703 \\
\hline 2004 & 59,846 & 58,136 & 53,057 & 50,111 & 2,946 & 5,078 & 1,710 \\
\hline 2005 & 60,238 & 58,514 & 53,419 & 50,466 & 2,954 & 5,095 & 1,724 \\
\hline 2006 & 60,587 & 58,846 & 53,729 & 50,763 & 2,966 & 5,117 & 1,742 \\
\hline 2007 & 60,975 & 59,216 & 54,072 & 51,092 & 2,980 & 5,144 & 1,759 \\
\hline 2007 by age $g$ & & & & & & & \\
\hline $0-4$ & 5.9 & 5.9 & 5.9 & 5.9 & 5.5 & 5.3 & 6.6 \\
\hline 5-15 & 13.0 & 12.9 & 13.0 & 13.0 & 13.2 & 12.5 & 15.1 \\
\hline $16-44$ & 40.1 & 40.1 & 40.2 & 40.3 & 37.4 & 39.3 & 41.2 \\
\hline $45-64 \mathrm{M} / 59 \mathrm{~F}$ & 22.0 & 22.1 & 21.9 & 21.9 & 22.9 & 23.4 & 20.7 \\
\hline $65 \mathrm{M} / 60 \mathrm{~F}-74$ & 11.2 & 11.3 & 11.2 & 11.1 & 12.5 & 11.9 & 10.1 \\
\hline 75 and over & 7.7 & 7.8 & 7.8 & 7.8 & 8.5 & 7.5 & 6.3 \\
\hline \multicolumn{8}{|l|}{ Projections ${ }^{1}$} \\
\hline 2006 & 60,587 & 58,846 & 53,729 & 50,763 & 2,966 & 5,117 & 1,742 \\
\hline 2011 & 62,761 & 60,950 & 55,744 & 52,706 & 3,038 & 5,206 & 1,812 \\
\hline 2016 & 64,975 & 63,107 & 57,837 & 54,724 & 3,113 & 5,270 & 1,868 \\
\hline 2021 & 67,191 & 65,269 & 59,943 & 56,757 & 3,186 & 5,326 & 1,922 \\
\hline 2026 & 69,260 & 67,294 & 61,931 & 58,682 & 3,248 & 5,363 & 1,966 \\
\hline 2031 & 71,100 & 69,101 & 63,727 & 60,432 & 3,296 & 5,374 & 1,999 \\
\hline \multicolumn{8}{|c|}{2031 by age group (percentages) } \\
\hline $0-4$ & 5.5 & 5.5 & 5.6 & 5.6 & 5.1 & 4.7 & 5.7 \\
\hline 5-15 & 12.4 & 12.4 & 12.5 & 12.5 & 12.1 & 11.2 & 13.4 \\
\hline $16-44$ & 36.4 & 36.4 & 36.6 & 36.8 & 33.7 & 34.3 & 35.5 \\
\hline $45-64^{2}$ & 23.4 & 23.4 & 23.3 & 23.3 & 23.5 & 24.4 & 23.9 \\
\hline $65-74^{2}$ & 10.6 & 10.6 & 10.5 & 10.4 & 12.0 & 12.4 & 10.7 \\
\hline 75 and over & 11.6 & 11.6 & 11.5 & 11.4 & 13.7 & 12.9 & 10.9 \\
\hline
\end{tabular}

Note: Figures may not add exactly due to rounding.

1 National projections based on mid-2006 population estimates.

2 Between 2010 and 2020, state pension age will change from 65 years for men and 60 years for women to 65 years for both sexes.

Between 2024 and 2026, state pension age will increase from 65 years to 66 years for both men and women. 
Population: subnational

Government Office Regions of England

\begin{tabular}{|c|c|c|c|c|c|c|c|c|c|}
\hline Mid-year & $\begin{array}{l}\text { North } \\
\text { East }\end{array}$ & $\begin{array}{l}\text { North } \\
\text { West }\end{array}$ & $\begin{array}{l}\text { Yorkshire } \\
\text { and The } \\
\text { Humber }\end{array}$ & $\begin{array}{c}\text { East } \\
\text { Midlands }\end{array}$ & $\begin{array}{c}\text { West } \\
\text { Midlands }\end{array}$ & East & London & $\begin{array}{c}\text { South } \\
\text { East }\end{array}$ & $\begin{array}{l}\text { South } \\
\text { West }\end{array}$ \\
\hline \multicolumn{10}{|l|}{ Estimates } \\
\hline 1971 & 2,679 & 7,108 & 4,902 & 3,652 & 5,146 & 4,454 & 7,529 & 6,830 & 4,112 \\
\hline 1976 & 2,671 & 7,043 & 4,924 & 3,774 & 5,178 & 4,672 & 7,089 & 7,029 & 4,280 \\
\hline 1981 & 2,636 & 6,940 & 4,918 & 3,853 & 5,187 & 4,854 & 6,806 & 7,245 & 4,381 \\
\hline 1986 & 2,594 & 6,833 & 4,884 & 3,908 & 5,180 & 4,999 & 6,774 & 7,468 & 4,548 \\
\hline 1991 & 2,587 & 6,843 & 4,936 & 4,011 & 5,230 & 5,121 & 6,829 & 7,629 & 4,688 \\
\hline 1993 & 2,594 & 6,847 & 4,954 & 4,056 & 5,246 & 5,154 & 6,844 & 7,673 & 4,734 \\
\hline 1994 & 2,589 & 6,839 & 4,960 & 4,072 & 5,249 & 5,178 & 6,874 & 7,712 & 4,757 \\
\hline 1995 & 2,583 & 6,828 & 4,961 & 4,092 & 5,257 & 5,206 & 6,913 & 7,763 & 4,782 \\
\hline 1996 & 2,576 & 6,810 & 4,961 & 4,108 & 5,263 & 5,233 & 6,974 & 7,800 & 4,793 \\
\hline 1997 & 2,568 & 6,794 & 4,958 & 4,120 & 5,262 & 5,267 & 7,015 & 7,853 & 4,827 \\
\hline 1998 & 2,561 & 6,792 & 4,958 & 4,133 & 5,271 & 5,302 & 7,065 & 7,889 & 4,849 \\
\hline 1999 & 2,550 & 6,773 & 4,956 & 4,152 & 5,272 & 5,339 & 7,154 & 7,955 & 4,881 \\
\hline 2000 & 2,543 & 6,774 & 4,959 & 4,168 & 5,270 & 5,375 & 7,237 & 7,991 & 4,917 \\
\hline 2001 & 2,540 & 6,773 & 4,977 & 4,190 & 5,281 & 5,400 & 7,322 & 8,023 & 4,943 \\
\hline 2002 & 2,541 & 6,778 & 5,002 & 4,222 & 5,295 & 5,433 & 7,362 & 8,047 & 4,973 \\
\hline 2003 & 2,541 & 6,800 & 5,028 & 4,254 & 5,312 & 5,475 & 7,364 & 8,087 & 5,005 \\
\hline 2004 & 2,542 & 6,820 & 5,064 & 4,291 & 5,327 & 5,511 & 7,389 & 8,125 & 5,042 \\
\hline 2005 & 2,550 & 6,840 & 5,108 & 4,328 & 5,351 & 5,563 & 7,456 & 8,185 & 5,087 \\
\hline 2006 & 2,556 & 6,853 & 5,142 & 4,364 & 5,367 & 5,607 & 7,512 & 8,238 & 5,124 \\
\hline 2007 & 2,564 & 6,864 & 5,177 & 4,400 & 5,382 & 5,661 & 7,557 & 8,309 & 5,178 \\
\hline \multicolumn{10}{|c|}{2007 by age group (percentages) } \\
\hline $0-4$ & 5.5 & 5.9 & 5.8 & 5.6 & 6.1 & 5.9 & 7.0 & 5.8 & 5.2 \\
\hline $5-15$ & 12.6 & 13.2 & 13.0 & 12.9 & 13.4 & 13.2 & 12.2 & 13.2 & 12.5 \\
\hline $16-44$ & 38.9 & 39.4 & 40.3 & 39.4 & 39.1 & 38.6 & 48.3 & 38.9 & 37.1 \\
\hline $45-64 \mathrm{M} / 59 \mathrm{~F}$ & 23.2 & 22.3 & 22.0 & 22.7 & 22.0 & 22.4 & 18.6 & 22.5 & 22.9 \\
\hline $65 \mathrm{M} / 60 \mathrm{~F}-74$ & 11.9 & 11.5 & 11.3 & 11.6 & 11.6 & 11.7 & 8.1 & 11.3 & 12.6 \\
\hline 75 and over & 8.0 & 7.7 & 7.6 & 7.8 & 7.9 & 8.2 & 5.7 & 8.3 & 9.5 \\
\hline \multicolumn{10}{|l|}{$\mathrm{ns}^{1}$} \\
\hline 2006 & 2,556 & 6,853 & 5,142 & 4,364 & 5,367 & 5,607 & 7,512 & 8,238 & 5,124 \\
\hline 2011 & 2,594 & 7,014 & 5,377 & 4,591 & 5,506 & 5,890 & 7,817 & 8,550 & 5,368 \\
\hline 2016 & 2,638 & 7,193 & 5,621 & 4,825 & 5,662 & 6,179 & 8,114 & 8,871 & 5,620 \\
\hline 2021 & 2,685 & 7,377 & 5,866 & 5,060 & 5,824 & 6,471 & 8,390 & 9,202 & 5,882 \\
\hline 2026 & 2,730 & 7,546 & 6,101 & 5,286 & 5,977 & 6,747 & 8,633 & 9,523 & 6,139 \\
\hline 2031 & 2,769 & 7,696 & 6,319 & 5,491 & 6,114 & 6,997 & 8,858 & 9,814 & 6,374 \\
\hline 2031 by age group & & & & & & & & & \\
\hline $0-4$ & 5.2 & 5.5 & 5.6 & 5.3 & 5.9 & 5.5 & 6.7 & 5.5 & 5.0 \\
\hline $5-15$ & 12.1 & 12.6 & 12.5 & 12.3 & 13.2 & 12.6 & 12.6 & 12.7 & 11.8 \\
\hline $16-44$ & 35.6 & 36.3 & 37.7 & 35.6 & 35.7 & 34.9 & 43.7 & 35.1 & 33.9 \\
\hline $45-64^{2}$ & 23.0 & 23.2 & 23.0 & 23.8 & 22.8 & 23.7 & 22.9 & 23.6 & 23.7 \\
\hline $65-74^{2}$ & 11.7 & 10.9 & 10.2 & 11.0 & 10.5 & 10.9 & 7.4 & 10.8 & 11.8 \\
\hline 75 and over & 12.4 & 11.5 & 10.9 & 12.0 & 11.9 & 12.4 & 6.7 & 12.4 & 13.9 \\
\hline
\end{tabular}

Note: Figures may not add exactly due to rounding.

1 These projections are based on the 2006 population estimates and are consistent with the 2006-based national projections produced by the Office for National Statistics.

2 Between 2010 and 2020, state pension age will change from 65 years for men and 60 years for women to 65 years for both sexes.

Between 2024 and 2026, state pension age will increase from 65 years to 66 years for both men and women. 


\begin{tabular}{|c|c|c|c|c|c|c|c|c|c|c|c|c|c|c|c|c|}
\hline Table 1.4 & \multicolumn{16}{|c|}{ Population: age and sex } \\
\hline \multicolumn{15}{|c|}{ Constituent countries of the United Kingdom } & \multicolumn{2}{|c|}{ Numbers (thousands) } \\
\hline & & \multicolumn{15}{|c|}{ Age group } \\
\hline Mid-year & All ages & Under 1 & $1-4$ & $5-14$ & $15-24$ & $25-34$ & $35-44$ & $45-59$ & $60-64$ & $65-74$ & $75-84$ & $85-89$ & $\begin{array}{c}90 \text { and } \\
\text { over }\end{array}$ & $\begin{array}{c}\text { Under } \\
16\end{array}$ & \begin{tabular}{|c|}
$16-$ \\
$64 \mathrm{M} / 59 \mathrm{~F}^{1}$
\end{tabular} & $\begin{array}{l}65 \mathrm{M} / 60 \mathrm{~F}^{1} \\
\text { and over }\end{array}$ \\
\hline $\begin{array}{l}\text { United Kingd } \\
\text { Persons } \\
1981 \\
1986 \\
1991 \\
1996\end{array}$ & $\begin{array}{l}56,357 \\
56,684 \\
57,439 \\
58,164\end{array}$ & $\begin{array}{l}730 \\
748 \\
790 \\
719\end{array}$ & $\begin{array}{l}2,726 \\
2,886 \\
3,077 \\
3,019\end{array}$ & $\begin{array}{l}8,147 \\
7,143 \\
7,141 \\
7,544\end{array}$ & $\begin{array}{l}9,019 \\
9,200 \\
8,168 \\
7,231\end{array}$ & $\begin{array}{l}8,010 \\
8,007 \\
8,898 \\
9,131\end{array}$ & $\begin{array}{l}6,774 \\
7,711 \\
7,918 \\
7,958\end{array}$ & $\begin{array}{r}9,540 \\
9,212 \\
9,500 \\
10,553\end{array}$ & $\begin{array}{l}2,935 \\
3,069 \\
2,888 \\
2,785\end{array}$ & $\begin{array}{l}5,195 \\
5,020 \\
5,067 \\
5,066\end{array}$ & $\begin{array}{l}2,677 \\
2,971 \\
3,119 \\
3,129\end{array}$ & $\begin{array}{l}716 \\
626 \\
711\end{array}$ & $\begin{array}{r}\ddot{*} \\
24 \ddot{8} \\
317\end{array}$ & $\begin{array}{l}12,543 \\
11,645 \\
11,685 \\
12,018\end{array}$ & $\begin{array}{l}33,780 \\
34,725 \\
35,197 \\
35,498\end{array}$ & $\begin{array}{l}10,035 \\
10,313 \\
10,557 \\
10,649\end{array}$ \\
\hline $\begin{array}{l}2000 \\
2001 \\
2002 \\
2003\end{array}$ & $\begin{array}{l}58,886 \\
59,113 \\
59,323 \\
59,557\end{array}$ & $\begin{array}{l}682 \\
663 \\
661 \\
680\end{array}$ & $\begin{array}{l}2,869 \\
2,819 \\
2,753 \\
2,706\end{array}$ & $\begin{array}{l}7,652 \\
7,624 \\
7,603 \\
7,546\end{array}$ & $\begin{array}{l}7,139 \\
7,261 \\
7,400 \\
7,573\end{array}$ & $\begin{array}{l}8,646 \\
8,475 \\
8,264 \\
8,084\end{array}$ & $\begin{array}{l}8,678 \\
8,846 \\
9,004 \\
9,105\end{array}$ & $\begin{array}{l}11,011 \\
11,168 \\
11,307 \\
11,412\end{array}$ & $\begin{array}{l}2,900 \\
2,884 \\
2,892 \\
2,949\end{array}$ & $\begin{array}{l}4,940 \\
4,947 \\
4,967 \\
5,001\end{array}$ & $\begin{array}{l}3,249 \\
3,296 \\
3,344 \\
3,398\end{array}$ & $\begin{array}{l}755 \\
753 \\
738 \\
706\end{array}$ & $\begin{array}{l}364 \\
377 \\
388 \\
399\end{array}$ & $\begin{array}{l}11,959 \\
11,863 \\
11,785 \\
11,720\end{array}$ & $\begin{array}{l}36,138 \\
36,406 \\
36,622 \\
36,826\end{array}$ & $\begin{array}{l}10,788 \\
10,845 \\
10,916 \\
11,012\end{array}$ \\
\hline $\begin{array}{l}2004 \\
2005 \\
2006 \\
2007\end{array}$ & $\begin{array}{l}59,846 \\
60,238 \\
60,587 \\
60,975\end{array}$ & $\begin{array}{l}705 \\
716 \\
732 \\
756\end{array}$ & $\begin{array}{l}2,686 \\
2,713 \\
2,765 \\
2,837\end{array}$ & $\begin{array}{l}7,475 \\
7,373 \\
7,241 \\
7,128\end{array}$ & $\begin{array}{l}7,739 \\
7,886 \\
8,020 \\
8,156\end{array}$ & $\begin{array}{l}7,954 \\
7,935 \\
7,896 \\
7,859\end{array}$ & $\begin{array}{l}9,185 \\
9,245 \\
9,262 \\
9,248\end{array}$ & $\begin{array}{l}11,507 \\
11,616 \\
11,744 \\
11,728\end{array}$ & $\begin{array}{l}3,027 \\
3,114 \\
3,240 \\
3,483\end{array}$ & $\begin{array}{l}5,028 \\
5,046 \\
5,029 \\
5,058\end{array}$ & $\begin{array}{l}3,431 \\
3,420 \\
3,416 \\
3,424\end{array}$ & $\begin{array}{l}702 \\
755 \\
820 \\
873\end{array}$ & $\begin{array}{l}409 \\
419 \\
423 \\
425\end{array}$ & $\begin{array}{l}11,645 \\
11,589 \\
11,537 \\
11,509\end{array}$ & $\begin{array}{l}37,083 \\
37,418 \\
37,707 \\
37,904\end{array}$ & $\begin{array}{l}11,117 \\
11,232 \\
11,344 \\
11,562\end{array}$ \\
\hline $\begin{array}{l}\text { Males } \\
1981 \\
1986 \\
1991 \\
1996\end{array}$ & $\begin{array}{l}27,412 \\
27,542 \\
27,909 \\
28,287\end{array}$ & $\begin{array}{l}374 \\
384 \\
403 \\
369\end{array}$ & $\begin{array}{l}1,400 \\
1,478 \\
1,572 \\
1,547\end{array}$ & $\begin{array}{l}4,184 \\
3,664 \\
3,655 \\
3,857\end{array}$ & $\begin{array}{l}4,596 \\
4,663 \\
4,146 \\
3,652\end{array}$ & $\begin{array}{l}4,035 \\
4,022 \\
4,432 \\
4,540\end{array}$ & $\begin{array}{l}3,409 \\
3,864 \\
3,949 \\
3,954\end{array}$ & $\begin{array}{l}4,711 \\
4,572 \\
4,732 \\
5,244\end{array}$ & $\begin{array}{l}1,376 \\
1,463 \\
1,390 \\
1,360\end{array}$ & $\begin{array}{l}2,264 \\
2,206 \\
2,272 \\
2,311\end{array}$ & $\begin{array}{r}922 \\
1,060 \\
1,146 \\
1,187\end{array}$ & $\begin{array}{l}166 \\
166 \\
201\end{array}$ & $\begin{array}{c}. . \\
4 \ddot{6} \\
65\end{array}$ & $\begin{array}{l}6,439 \\
5,968 \\
5,976 \\
6,148\end{array}$ & $\begin{array}{l}17,646 \\
18,142 \\
18,303 \\
18,375\end{array}$ & $\begin{array}{l}3,327 \\
3,432 \\
3,630 \\
3,764\end{array}$ \\
\hline $\begin{array}{l}2000 \\
2001 \\
2002 \\
2003\end{array}$ & $\begin{array}{l}28,690 \\
28,832 \\
28,964 \\
29,109\end{array}$ & $\begin{array}{l}350 \\
338 \\
338 \\
349\end{array}$ & $\begin{array}{l}1,469 \\
1,445 \\
1,408 \\
1,384\end{array}$ & $\begin{array}{l}3,920 \\
3,906 \\
3,897 \\
3,868\end{array}$ & $\begin{array}{l}3,606 \\
3,672 \\
3,758 \\
3,855\end{array}$ & $\begin{array}{l}4,292 \\
4,215 \\
4,114 \\
4,024\end{array}$ & $\begin{array}{l}4,298 \\
4,382 \\
4,462 \\
4,514\end{array}$ & $\begin{array}{l}5,457 \\
5,534 \\
5,594 \\
5,646\end{array}$ & $\begin{array}{l}1,420 \\
1,412 \\
1,414 \\
1,440\end{array}$ & $\begin{array}{l}2,294 \\
2,308 \\
2,325 \\
2,347\end{array}$ & $\begin{array}{l}1,278 \\
1,308 \\
1,338 \\
1,369\end{array}$ & $\begin{array}{l}225 \\
227 \\
226 \\
219\end{array}$ & $\begin{array}{l}81 \\
85 \\
89 \\
94\end{array}$ & $\begin{array}{l}6,128 \\
6,077 \\
6,037 \\
6,006\end{array}$ & $\begin{array}{l}18,685 \\
18,827 \\
18,949 \\
19,075\end{array}$ & $\begin{array}{l}3,878 \\
3,928 \\
3,978 \\
4,028\end{array}$ \\
\hline $\begin{array}{l}2004 \\
2005 \\
2006 \\
2007\end{array}$ & $\begin{array}{l}29,278 \\
29,497 \\
29,694 \\
29,916\end{array}$ & $\begin{array}{l}362 \\
367 \\
374 \\
387\end{array}$ & $\begin{array}{l}1,376 \\
1,389 \\
1,416 \\
1,453\end{array}$ & $\begin{array}{l}3,832 \\
3,781 \\
3,709 \\
3,649\end{array}$ & $\begin{array}{l}3,953 \\
4,030 \\
4,108 \\
4,193\end{array}$ & $\begin{array}{l}3,960 \\
3,952 \\
3,940 \\
3,936\end{array}$ & $\begin{array}{l}4,546 \\
4,581 \\
4,586 \\
4,578\end{array}$ & $\begin{array}{l}5,691 \\
5,745 \\
5,804 \\
5,786\end{array}$ & $\begin{array}{l}1,479 \\
1,522 \\
1,584 \\
1,701\end{array}$ & $\begin{array}{l}2,365 \\
2,380 \\
2,379 \\
2,398\end{array}$ & $\begin{array}{l}1,392 \\
1,400 \\
1,413 \\
1,432\end{array}$ & $\begin{array}{l}223 \\
247 \\
273 \\
295\end{array}$ & $\begin{array}{r}98 \\
103 \\
106 \\
108\end{array}$ & $\begin{array}{l}5,971 \\
5,941 \\
5,912 \\
5,895\end{array}$ & $\begin{array}{l}19,229 \\
19,426 \\
19,611 \\
19,789\end{array}$ & $\begin{array}{l}4,078 \\
4,130 \\
4,171 \\
4,233\end{array}$ \\
\hline $\begin{array}{l}\text { Females } \\
1981 \\
1986 \\
1991 \\
1996\end{array}$ & $\begin{array}{l}28,946 \\
29,142 \\
29,530 \\
29,877\end{array}$ & $\begin{array}{l}356 \\
364 \\
387 \\
350\end{array}$ & $\begin{array}{l}1,327 \\
1,408 \\
1,505 \\
1,472\end{array}$ & $\begin{array}{l}3,963 \\
3,480 \\
3,487 \\
3,687\end{array}$ & $\begin{array}{l}4,423 \\
4,538 \\
4,021 \\
3,579\end{array}$ & $\begin{array}{l}3,975 \\
3,985 \\
4,466 \\
4,591\end{array}$ & $\begin{array}{l}3,365 \\
3,847 \\
3,968 \\
4,005\end{array}$ & $\begin{array}{l}4,829 \\
4,639 \\
4,769 \\
5,309\end{array}$ & $\begin{array}{l}1,559 \\
1,606 \\
1,498 \\
1,426\end{array}$ & $\begin{array}{r}2,931 \\
2,814 \\
2,795 \\
2,755\end{array}$ & $\begin{array}{l}1,756 \\
1,911 \\
1,972 \\
1,942\end{array}$ & $\begin{array}{l}550 \\
460 \\
509\end{array}$ & $\begin{array}{r}\ddot{*} \\
20 \ddot{2} \\
252\end{array}$ & $\begin{array}{l}6,104 \\
5,678 \\
5,709 \\
5,870\end{array}$ & $\begin{array}{l}16,134 \\
16,583 \\
16,894 \\
17,123\end{array}$ & $\begin{array}{l}6,708 \\
6,881 \\
6,927 \\
6,885\end{array}$ \\
\hline $\begin{array}{l}2000 \\
2001 \\
2002 \\
2003\end{array}$ & $\begin{array}{l}30,196 \\
30,281 \\
30,359 \\
30,449\end{array}$ & $\begin{array}{l}333 \\
324 \\
323 \\
331\end{array}$ & $\begin{array}{l}1,399 \\
1,375 \\
1,346 \\
1,322\end{array}$ & $\begin{array}{l}3,732 \\
3,718 \\
3,706 \\
3,678\end{array}$ & $\begin{array}{l}3,533 \\
3,589 \\
3,642 \\
3,718\end{array}$ & $\begin{array}{l}4,353 \\
4,260 \\
4,150 \\
4,060\end{array}$ & $\begin{array}{l}4,380 \\
4,465 \\
4,542 \\
4,590\end{array}$ & $\begin{array}{l}5,554 \\
5,634 \\
5,713 \\
5,766\end{array}$ & $\begin{array}{l}1,481 \\
1,473 \\
1,478 \\
1,509\end{array}$ & $\begin{array}{l}2,646 \\
2,640 \\
2,642 \\
2,654\end{array}$ & $\begin{array}{l}1,971 \\
1,987 \\
2,006 \\
2,029\end{array}$ & $\begin{array}{l}530 \\
526 \\
513 \\
487\end{array}$ & $\begin{array}{l}283 \\
292 \\
299 \\
305\end{array}$ & $\begin{array}{l}5,832 \\
5,786 \\
5,748 \\
5,714\end{array}$ & $\begin{array}{l}17,453 \\
17,579 \\
17,673 \\
17,751\end{array}$ & $\begin{array}{l}6,911 \\
6,917 \\
6,938 \\
6,984\end{array}$ \\
\hline $\begin{array}{l}2004 \\
2005 \\
2006 \\
2007\end{array}$ & $\begin{array}{l}30,568 \\
30,741 \\
30,893 \\
31,059\end{array}$ & $\begin{array}{l}343 \\
349 \\
357 \\
368\end{array}$ & $\begin{array}{l}1,310 \\
1,324 \\
1,349 \\
1,383\end{array}$ & $\begin{array}{l}3,642 \\
3,592 \\
3,532 \\
3,480\end{array}$ & $\begin{array}{l}3,785 \\
3,856 \\
3,912 \\
3,963\end{array}$ & $\begin{array}{l}3,993 \\
3,983 \\
3,956 \\
3,924\end{array}$ & $\begin{array}{l}4,639 \\
4,663 \\
4,675 \\
4,670\end{array}$ & $\begin{array}{l}5,816 \\
5,871 \\
5,940 \\
5,942\end{array}$ & $\begin{array}{l}1,548 \\
1,591 \\
1,656 \\
1,782\end{array}$ & $\begin{array}{l}2,662 \\
2,666 \\
2,650 \\
2,660\end{array}$ & $\begin{array}{l}2,040 \\
2,020 \\
2,002 \\
1,992\end{array}$ & $\begin{array}{l}479 \\
509 \\
547 \\
578\end{array}$ & $\begin{array}{l}310 \\
316 \\
317 \\
317\end{array}$ & $\begin{array}{l}5,674 \\
5,647 \\
5,625 \\
5,615\end{array}$ & $\begin{array}{l}17,854 \\
17,992 \\
18,096 \\
18,116\end{array}$ & $\begin{array}{l}7,039 \\
7,102 \\
7,172 \\
7,329\end{array}$ \\
\hline $\begin{array}{l}\text { England an } \\
\text { Persons } \\
1981 \\
1986 \\
1991 \\
1996\end{array}$ & $\begin{array}{l}49,634 \\
49,999 \\
50,748 \\
51,410\end{array}$ & $\begin{array}{l}634 \\
654 \\
698 \\
637\end{array}$ & $\begin{array}{l}2,372 \\
2,522 \\
2,713 \\
2,668\end{array}$ & $\begin{array}{l}7,085 \\
6,226 \\
6,248 \\
6,636\end{array}$ & $\begin{array}{l}7,873 \\
8,061 \\
7,165 \\
6,336\end{array}$ & $\begin{array}{l}7,086 \\
7,052 \\
7,862 \\
8,076\end{array}$ & $\begin{array}{l}5,996 \\
6,856 \\
7,022 \\
7,017\end{array}$ & $\begin{array}{l}8,433 \\
8,136 \\
8,407 \\
9,363\end{array}$ & $\begin{array}{l}2,607 \\
2,725 \\
2,553 \\
2,457\end{array}$ & $\begin{array}{l}4,619 \\
4,470 \\
4,506 \\
4,496\end{array}$ & $\begin{array}{l}2,388 \\
2,655 \\
2,790 \\
2,801\end{array}$ & $\begin{array}{l}383 \\
461 \\
561 \\
639\end{array}$ & $\begin{array}{l}157 \\
182 \\
223 \\
285\end{array}$ & $\begin{array}{l}10,910 \\
10,161 \\
10,247 \\
10,584\end{array}$ & $\begin{array}{l}29,796 \\
30,647 \\
31,100 \\
31,353\end{array}$ & $\begin{array}{l}8,928 \\
9,190 \\
9,400 \\
9,474\end{array}$ \\
\hline $\begin{array}{l}2000 \\
2001 \\
2002 \\
2003\end{array}$ & $\begin{array}{l}52,140 \\
52,360 \\
52,572 \\
52,797\end{array}$ & $\begin{array}{l}607 \\
589 \\
589 \\
607\end{array}$ & $\begin{array}{l}2,544 \\
2,502 \\
2,445 \\
2,404\end{array}$ & $\begin{array}{l}6,757 \\
6,740 \\
6,728 \\
6,682\end{array}$ & $\begin{array}{l}6,275 \\
6,387 \\
6,518 \\
6,679\end{array}$ & $\begin{array}{l}7,682 \\
7,536 \\
7,357 \\
7,203\end{array}$ & $\begin{array}{l}7,661 \\
7,816 \\
7,964 \\
8,058\end{array}$ & $\begin{array}{r}9,764 \\
9,898 \\
10,018 \\
10,104\end{array}$ & $\begin{array}{l}2,564 \\
2,549 \\
2,555 \\
2,606\end{array}$ & $\begin{array}{l}4,372 \\
4,377 \\
4,394 \\
4,422\end{array}$ & $\begin{array}{l}2,907 \\
2,947 \\
2,989 \\
3,037\end{array}$ & $\begin{array}{l}680 \\
677 \\
664 \\
634\end{array}$ & $\begin{array}{l}328 \\
340 \\
351 \\
360\end{array}$ & $\begin{array}{l}10,572 \\
10,495 \\
10,437 \\
10,388\end{array}$ & $\begin{array}{l}31,977 \\
32,226 \\
32,435 \\
32,626\end{array}$ & $\begin{array}{l}9,591 \\
9,639 \\
9,700 \\
9,783\end{array}$ \\
\hline $\begin{array}{l}2004 \\
2005 \\
2006 \\
2007\end{array}$ & $\begin{array}{l}53,057 \\
53,419 \\
53,729 \\
54,072\end{array}$ & $\begin{array}{l}629 \\
639 \\
653 \\
675\end{array}$ & $\begin{array}{l}2,390 \\
2,415 \\
2,462 \\
2,528\end{array}$ & $\begin{array}{l}6,618 \\
6,528 \\
6,412 \\
6,314\end{array}$ & $\begin{array}{l}6,836 \\
6,974 \\
7,095 \\
7,219\end{array}$ & $\begin{array}{l}7,090 \\
7,078 \\
7,040 \\
6,999\end{array}$ & $\begin{array}{l}8,133 \\
8,194 \\
8,213 \\
8,209\end{array}$ & $\begin{array}{l}10,177 \\
10,264 \\
10,369 \\
10,347\end{array}$ & $\begin{array}{l}2,675 \\
2,757 \\
2,874 \\
3,092\end{array}$ & $\begin{array}{l}4,445 \\
4,461 \\
4,444 \\
4,468\end{array}$ & $\begin{array}{l}3,063 \\
3,052 \\
3,045 \\
3,049\end{array}$ & $\begin{array}{l}632 \\
680 \\
740 \\
787\end{array}$ & $\begin{array}{l}370 \\
379 \\
382 \\
385\end{array}$ & $\begin{array}{l}10,326 \\
10,278 \\
10,235 \\
10,212\end{array}$ & $\begin{array}{l}32,856 \\
33,164 \\
33,417 \\
33,588\end{array}$ & $\begin{array}{r}9,875 \\
9,977 \\
10,077 \\
10,271\end{array}$ \\
\hline $\begin{array}{l}\text { Males } \\
1981 \\
1986 \\
1991 \\
1996\end{array}$ & $\begin{array}{l}24,160 \\
24,311 \\
24,681 \\
25,030\end{array}$ & $\begin{array}{l}324 \\
335 \\
356 \\
327\end{array}$ & $\begin{array}{l}1,218 \\
1,292 \\
1,385 \\
1,368\end{array}$ & $\begin{array}{l}3,639 \\
3,194 \\
3,198 \\
3,393\end{array}$ & $\begin{array}{l}4,011 \\
4,083 \\
3,638 \\
3,202\end{array}$ & $\begin{array}{l}3,569 \\
3,542 \\
3,920 \\
4,020\end{array}$ & $\begin{array}{l}3,024 \\
3,438 \\
3,504 \\
3,489\end{array}$ & $\begin{array}{l}4,178 \\
4,053 \\
4,199 \\
4,659\end{array}$ & $\begin{array}{l}1,227 \\
1,302 \\
1,234 \\
1,205\end{array}$ & $\begin{array}{l}2,020 \\
1,972 \\
2,027 \\
2,059\end{array}$ & $\begin{array}{r}825 \\
951 \\
1,029 \\
1,067\end{array}$ & $\begin{array}{r}94 \\
115 \\
150 \\
182\end{array}$ & $\begin{array}{l}32 \\
35 \\
42 \\
59\end{array}$ & $\begin{array}{l}5,601 \\
5,208 \\
5,240 \\
5,416\end{array}$ & $\begin{array}{l}15,589 \\
16,031 \\
16,193 \\
16,247\end{array}$ & $\begin{array}{r}2,970 \\
3,072 \\
3,248 \\
3,367\end{array}$ \\
\hline $\begin{array}{l}2000 \\
2001 \\
2002 \\
2003\end{array}$ & $\begin{array}{l}25,438 \\
25,574 \\
25,704 \\
25,841\end{array}$ & $\begin{array}{l}311 \\
301 \\
301 \\
312\end{array}$ & $\begin{array}{l}1,303 \\
1,281 \\
1,249 \\
1,230\end{array}$ & $\begin{array}{l}3,462 \\
3,453 \\
3,448 \\
3,425\end{array}$ & $\begin{array}{l}3,172 \\
3,231 \\
3,311 \\
3,399\end{array}$ & $\begin{array}{l}3,823 \\
3,758 \\
3,672 \\
3,594\end{array}$ & $\begin{array}{l}3,802 \\
3,881 \\
3,957 \\
4,007\end{array}$ & $\begin{array}{l}4,842 \\
4,907 \\
4,958 \\
5,002\end{array}$ & $\begin{array}{l}1,259 \\
1,252 \\
1,253 \\
1,276\end{array}$ & $\begin{array}{l}2,040 \\
2,052 \\
2,067 \\
2,085\end{array}$ & $\begin{array}{l}1,148 \\
1,175 \\
1,202 \\
1,229\end{array}$ & $\begin{array}{l}204 \\
206 \\
204 \\
198\end{array}$ & $\begin{array}{l}73 \\
77 \\
81 \\
85\end{array}$ & $\begin{array}{l}5,416 \\
5,376 \\
5,346 \\
5,324\end{array}$ & $\begin{array}{l}16,556 \\
16,688 \\
16,804 \\
16,920\end{array}$ & $\begin{array}{l}3,466 \\
3,510 \\
3,554 \\
3,597\end{array}$ \\
\hline $\begin{array}{l}2004 \\
2005 \\
2006 \\
2007\end{array}$ & $\begin{array}{l}25,995 \\
26,197 \\
26,371 \\
26,569\end{array}$ & $\begin{array}{l}323 \\
327 \\
334 \\
346\end{array}$ & $\begin{array}{l}1,225 \\
1,237 \\
1,261 \\
1,295\end{array}$ & $\begin{array}{l}3,394 \\
3,348 \\
3,284 \\
3,231\end{array}$ & $\begin{array}{l}3,493 \\
3,565 \\
3,636 \\
3,715\end{array}$ & $\begin{array}{l}3,538 \\
3,530 \\
3,517 \\
3,508\end{array}$ & $\begin{array}{l}4,036 \\
4,073 \\
4,080 \\
4,076\end{array}$ & $\begin{array}{l}5,037 \\
5,080 \\
5,130 \\
5,110\end{array}$ & $\begin{array}{l}1,310 \\
1,351 \\
1,407 \\
1,511\end{array}$ & $\begin{array}{l}2,100 \\
2,113 \\
2,111 \\
2,127\end{array}$ & $\begin{array}{l}1,248 \\
1,256 \\
1,267 \\
1,283\end{array}$ & $\begin{array}{l}202 \\
224 \\
248 \\
268\end{array}$ & $\begin{array}{l}89 \\
94 \\
96 \\
99\end{array}$ & $\begin{array}{l}5,295 \\
5,270 \\
5,245 \\
5,230\end{array}$ & $\begin{array}{l}17,060 \\
17,241 \\
17,405 \\
17,563\end{array}$ & $\begin{array}{l}3,640 \\
3,685 \\
3,722 \\
3,775\end{array}$ \\
\hline $\begin{array}{l}\text { Females } \\
1981 \\
1986 \\
1991 \\
1996\end{array}$ & $\begin{array}{l}25,474 \\
25,687 \\
26,067 \\
26,381\end{array}$ & $\begin{array}{l}310 \\
319 \\
342 \\
310\end{array}$ & $\begin{array}{l}1,154 \\
1,231 \\
1,328 \\
1,300\end{array}$ & $\begin{array}{l}3,446 \\
3,032 \\
3,050 \\
3,243\end{array}$ & $\begin{array}{l}3,863 \\
3,978 \\
3,527 \\
3,134\end{array}$ & $\begin{array}{l}3,517 \\
3,509 \\
3,943 \\
4,056\end{array}$ & $\begin{array}{l}2,972 \\
3,418 \\
3,517 \\
3,528\end{array}$ & $\begin{array}{l}4,255 \\
4,083 \\
4,208 \\
4,704\end{array}$ & $\begin{array}{l}1,380 \\
1,422 \\
1,319 \\
1,252\end{array}$ & $\begin{array}{l}2,599 \\
2,498 \\
2,479 \\
2,437\end{array}$ & $\begin{array}{l}1,564 \\
1,704 \\
1,761 \\
1,734\end{array}$ & $\begin{array}{l}289 \\
346 \\
411 \\
457\end{array}$ & $\begin{array}{l}126 \\
148 \\
181 \\
227\end{array}$ & $\begin{array}{l}5,309 \\
4,953 \\
5,007 \\
5,168\end{array}$ & $\begin{array}{l}14,207 \\
14,616 \\
14,908 \\
15,106\end{array}$ & $\begin{array}{l}5,958 \\
6,118 \\
6,152 \\
6,107\end{array}$ \\
\hline $\begin{array}{l}2000 \\
2001 \\
2002 \\
2003\end{array}$ & $\begin{array}{l}26,702 \\
26,786 \\
26,868 \\
26,956\end{array}$ & $\begin{array}{l}296 \\
288 \\
287 \\
295\end{array}$ & $\begin{array}{l}1,241 \\
1,220 \\
1,195 \\
1,175\end{array}$ & $\begin{array}{l}3,296 \\
3,287 \\
3,280 \\
3,256\end{array}$ & $\begin{array}{l}3,103 \\
3,156 \\
3,207 \\
3,280\end{array}$ & $\begin{array}{l}3,859 \\
3,778 \\
3,685 \\
3,610\end{array}$ & $\begin{array}{l}3,859 \\
3,935 \\
4,007 \\
4,051\end{array}$ & $\begin{array}{l}4,923 \\
4,992 \\
5,060 \\
5,103\end{array}$ & $\begin{array}{l}1,304 \\
1,297 \\
1,302 \\
1,329\end{array}$ & $\begin{array}{l}2,332 \\
2,326 \\
2,328 \\
2,338\end{array}$ & $\begin{array}{l}1,758 \\
1,771 \\
1,787 \\
1,807\end{array}$ & $\begin{array}{l}476 \\
471 \\
460 \\
436\end{array}$ & $\begin{array}{l}255 \\
263 \\
270 \\
275\end{array}$ & $\begin{array}{l}5,155 \\
5,119 \\
5,091 \\
5,064\end{array}$ & $\begin{array}{l}15,421 \\
15,538 \\
15,631 \\
15,705\end{array}$ & $\begin{array}{l}6,126 \\
6,129 \\
6,146 \\
6,186\end{array}$ \\
\hline $\begin{array}{l}2004 \\
2005 \\
2006 \\
2007\end{array}$ & $\begin{array}{l}27,062 \\
27,223 \\
27,358 \\
27,503\end{array}$ & $\begin{array}{l}306 \\
312 \\
319 \\
329\end{array}$ & $\begin{array}{l}1,165 \\
1,178 \\
1,201 \\
1,233 \\
\end{array}$ & $\begin{array}{l}3,224 \\
3,180 \\
3,127 \\
3,082\end{array}$ & $\begin{array}{l}3,342 \\
3,409 \\
3,458 \\
3,505\end{array}$ & $\begin{array}{l}3,552 \\
3,548 \\
3,523 \\
3,490\end{array}$ & $\begin{array}{l}4,097 \\
4,121 \\
4,134 \\
4,132 \\
\end{array}$ & $\begin{array}{l}5,141 \\
5,183 \\
5,239 \\
5,237\end{array}$ & $\begin{array}{l}1,365 \\
1,406 \\
1,466 \\
1,581\end{array}$ & $\begin{array}{l}2,345 \\
2,348 \\
2,333 \\
2,342\end{array}$ & $\begin{array}{l}1,815 \\
1,796 \\
1,778 \\
1,767\end{array}$ & $\begin{array}{l}430 \\
456 \\
492 \\
520\end{array}$ & $\begin{array}{l}280 \\
285 \\
286 \\
286\end{array}$ & $\begin{array}{l}5,031 \\
5,008 \\
4,990 \\
4,982\end{array}$ & $\begin{array}{l}15,796 \\
15,922 \\
16,012 \\
16,026\end{array}$ & $\begin{array}{l}6,235 \\
6,292 \\
6,355 \\
6,496\end{array}$ \\
\hline
\end{tabular}

1 Between 2010 and 2020, state pension age will change from 65 years for men and 60 years for women to 65 years for both sexes. 


\begin{tabular}{|c|c|c|c|c|c|c|c|c|c|c|c|c|c|c|c|c|}
\hline $\begin{array}{l}\text { Table } 1.4 \\
\text { continued }\end{array}$ & \multicolumn{16}{|c|}{ Population: age and sex } \\
\hline \multicolumn{15}{|c|}{ Constituent countries of the United Kingdom } & \multicolumn{2}{|c|}{ Numbers (thousands) } \\
\hline & & \multicolumn{15}{|c|}{ Age group } \\
\hline Mid-year & All ages & Under 1 & $1-4$ & $5-14$ & $15-24$ & $25-34$ & $35-44$ & $45-59$ & $60-64$ & $65-74$ & $75-84$ & $85-89$ & $\begin{array}{c}90 \text { and } \\
\text { over }\end{array}$ & $\begin{array}{c}\text { Under } \\
16\end{array}$ & \begin{tabular}{|c|}
$16-$ \\
$64 \mathrm{M} / 59 \mathrm{~F}^{1}$
\end{tabular} & $\begin{array}{l}65 \mathrm{M} / 65 \mathrm{~F}^{1} \\
\text { and over }\end{array}$ \\
\hline $\begin{array}{l}\text { England } \\
\text { Persons } \\
1981 \\
1986 \\
1991 \\
1996\end{array}$ & $\begin{array}{l}46,821 \\
47,188 \\
47,875 \\
48,519\end{array}$ & $\begin{array}{l}598 \\
618 \\
660 \\
603\end{array}$ & $\begin{array}{l}2,235 \\
2,380 \\
2,560 \\
2,523\end{array}$ & $\begin{array}{l}6,678 \\
5,869 \\
5,885 \\
6,255\end{array}$ & $\begin{array}{l}7,440 \\
7,623 \\
6,772 \\
5,985\end{array}$ & $\begin{array}{l}6,703 \\
6,682 \\
7,460 \\
7,667\end{array}$ & $\begin{array}{l}5,663 \\
6,478 \\
6,633 \\
6,638\end{array}$ & $\begin{array}{l}7,948 \\
7,672 \\
7,920 \\
8,822\end{array}$ & $\begin{array}{l}2,449 \\
2,559 \\
2,399 \\
2,310\end{array}$ & $\begin{array}{l}4,347 \\
4,199 \\
4,222 \\
4,217\end{array}$ & $\begin{array}{l}2,249 \\
2,501 \\
2,626 \\
2,631\end{array}$ & $\begin{array}{l}362 \\
435 \\
529 \\
602\end{array}$ & $\begin{array}{l}149 \\
172 \\
210 \\
269\end{array}$ & $\begin{array}{r}10,285 \\
9,583 \\
9,658 \\
9,985\end{array}$ & $\begin{array}{l}28,133 \\
28,962 \\
29,390 \\
29,639\end{array}$ & $\begin{array}{l}8,403 \\
8,643 \\
8,827 \\
8,895\end{array}$ \\
\hline $\begin{array}{l}2000 \\
2001 \\
2002 \\
2003\end{array}$ & $\begin{array}{l}49,233 \\
49,450 \\
49,652 \\
49,866\end{array}$ & $\begin{array}{l}575 \\
558 \\
559 \\
576\end{array}$ & $\begin{array}{l}2,406 \\
2,366 \\
2,313 \\
2,275\end{array}$ & $\begin{array}{l}6,375 \\
6,359 \\
6,348 \\
6,305\end{array}$ & $\begin{array}{l}5,923 \\
6,032 \\
6,153 \\
6,304\end{array}$ & $\begin{array}{l}7,304 \\
7,171 \\
7,003 \\
6,859\end{array}$ & $\begin{array}{l}7,257 \\
7,407 \\
7,550 \\
7,641\end{array}$ & $\begin{array}{l}9,199 \\
9,327 \\
9,439 \\
9,522\end{array}$ & $\begin{array}{l}2,411 \\
2,395 \\
2,399 \\
2,445\end{array}$ & $\begin{array}{l}4,107 \\
4,113 \\
4,129 \\
4,155\end{array}$ & $\begin{array}{l}2,727 \\
2,764 \\
2,803 \\
2,850\end{array}$ & $\begin{array}{l}641 \\
638 \\
625 \\
596\end{array}$ & $\begin{array}{l}309 \\
321 \\
331 \\
340\end{array}$ & $\begin{array}{l}9,980 \\
9,908 \\
9,855 \\
9,812\end{array}$ & $\begin{array}{l}30,243 \\
30,487 \\
30,686 \\
30,867\end{array}$ & $\begin{array}{l}9,010 \\
9,055 \\
9,111 \\
9,188\end{array}$ \\
\hline $\begin{array}{l}2004 \\
2005 \\
2006 \\
2007\end{array}$ & $\begin{array}{l}50,111 \\
50,466 \\
50,763 \\
51,092\end{array}$ & $\begin{array}{l}597 \\
606 \\
620 \\
641\end{array}$ & $\begin{array}{l}2,262 \\
2,289 \\
2,335 \\
2,398\end{array}$ & $\begin{array}{l}6,245 \\
6,161 \\
6,051 \\
5,961\end{array}$ & $\begin{array}{l}6,450 \\
6,583 \\
6,696 \\
6,812\end{array}$ & $\begin{array}{l}6,751 \\
6,742 \\
6,708 \\
6,669\end{array}$ & $\begin{array}{l}7,712 \\
7,772 \\
7,793 \\
7,791\end{array}$ & $\begin{array}{l}9,591 \\
9,675 \\
9,777 \\
9,758\end{array}$ & $\begin{array}{l}2,509 \\
2,586 \\
2,697 \\
2,904\end{array}$ & $\begin{array}{l}4,175 \\
4,189 \\
4,171 \\
4,192\end{array}$ & $\begin{array}{l}2,875 \\
2,865 \\
2,860 \\
2,865\end{array}$ & $\begin{array}{l}593 \\
638 \\
695 \\
739\end{array}$ & $\begin{array}{l}349 \\
357 \\
360 \\
363\end{array}$ & $\begin{array}{l}9,755 \\
9,713 \\
9,674 \\
9,656\end{array}$ & $\begin{array}{l}31,083 \\
31,384 \\
31,627 \\
31,792\end{array}$ & $\begin{array}{l}9,273 \\
9,370 \\
9,462 \\
9,645\end{array}$ \\
\hline $\begin{array}{l}\text { Males } \\
1981 \\
1986 \\
1991 \\
1996\end{array}$ & $\begin{array}{l}22,795 \\
22,949 \\
23,291 \\
23,629\end{array}$ & $\begin{array}{l}306 \\
317 \\
336 \\
309\end{array}$ & $\begin{array}{l}1,147 \\
1,219 \\
1,307 \\
1,294\end{array}$ & $\begin{array}{l}3,430 \\
3,010 \\
3,011 \\
3,198\end{array}$ & $\begin{array}{l}3,790 \\
3,862 \\
3,439 \\
3,023\end{array}$ & $\begin{array}{l}3,377 \\
3,357 \\
3,721 \\
3,818\end{array}$ & $\begin{array}{l}2,856 \\
3,249 \\
3,311 \\
3,302\end{array}$ & $\begin{array}{l}3,938 \\
3,822 \\
3,957 \\
4,390\end{array}$ & $\begin{array}{l}1,154 \\
1,224 \\
1,159 \\
1,133\end{array}$ & $\begin{array}{l}1,902 \\
1,853 \\
1,900 \\
1,932\end{array}$ & $\begin{array}{r}777 \\
897 \\
970 \\
1,003\end{array}$ & $\begin{array}{l}89 \\
108 \\
141 \\
172\end{array}$ & $\begin{array}{l}30 \\
33 \\
39 \\
55\end{array}$ & $\begin{array}{l}5,280 \\
4,911 \\
4,938 \\
5,110\end{array}$ & $\begin{array}{l}14,717 \\
15,147 \\
15,302 \\
15,358\end{array}$ & $\begin{array}{l}2,798 \\
2,891 \\
3,050 \\
3,161\end{array}$ \\
\hline $\begin{array}{l}2000 \\
2001 \\
2002 \\
2003\end{array}$ & $\begin{array}{l}24,030 \\
24,166 \\
24,290 \\
24,419\end{array}$ & $\begin{array}{l}294 \\
285 \\
286 \\
296\end{array}$ & $\begin{array}{l}1,232 \\
1,212 \\
1,182 \\
1,163\end{array}$ & $\begin{array}{l}3,266 \\
3,257 \\
3,253 \\
3,232\end{array}$ & $\begin{array}{l}2,995 \\
3,053 \\
3,127 \\
3,209\end{array}$ & $\begin{array}{l}3,638 \\
3,580 \\
3,500 \\
3,425\end{array}$ & $\begin{array}{l}3,604 \\
3,681 \\
3,755 \\
3,803\end{array}$ & $\begin{array}{l}4,562 \\
4,624 \\
4,673 \\
4,715\end{array}$ & $\begin{array}{l}1,184 \\
1,176 \\
1,176 \\
1,197\end{array}$ & $\begin{array}{l}1,917 \\
1,928 \\
1,942 \\
1,958\end{array}$ & $\begin{array}{l}1,078 \\
1,103 \\
1,128 \\
1,154\end{array}$ & $\begin{array}{l}192 \\
194 \\
193 \\
186\end{array}$ & $\begin{array}{l}69 \\
73 \\
77 \\
80\end{array}$ & $\begin{array}{l}5,113 \\
5,075 \\
5,047 \\
5,028\end{array}$ & $\begin{array}{l}15,661 \\
15,793 \\
15,904 \\
16,012\end{array}$ & $\begin{array}{l}3,256 \\
3,298 \\
3,339 \\
3,379\end{array}$ \\
\hline $\begin{array}{l}2004 \\
2005 \\
2006 \\
2007\end{array}$ & $\begin{array}{l}24,563 \\
24,758 \\
24,926 \\
25,114\end{array}$ & $\begin{array}{l}306 \\
310 \\
317 \\
328\end{array}$ & $\begin{array}{l}1,159 \\
1,172 \\
1,196 \\
1,228\end{array}$ & $\begin{array}{l}3,202 \\
3,160 \\
3,100 \\
3,050\end{array}$ & $\begin{array}{l}3,297 \\
3,365 \\
3,432 \\
3,506\end{array}$ & $\begin{array}{l}3,371 \\
3,365 \\
3,353 \\
3,345\end{array}$ & $\begin{array}{l}3,831 \\
3,868 \\
3,875 \\
3,874\end{array}$ & $\begin{array}{l}4,748 \\
4,791 \\
4,839 \\
4,821\end{array}$ & $\begin{array}{l}1,228 \\
1,267 \\
1,320 \\
1,418\end{array}$ & $\begin{array}{l}1,972 \\
1,984 \\
1,981 \\
1,995\end{array}$ & $\begin{array}{l}1,172 \\
1,179 \\
1,190 \\
1,205\end{array}$ & $\begin{array}{l}190 \\
210 \\
233 \\
251\end{array}$ & $\begin{array}{l}84 \\
88 \\
91 \\
93\end{array}$ & $\begin{array}{l}5,001 \\
4,979 \\
4,957 \\
4,944\end{array}$ & $\begin{array}{l}16,143 \\
16,317 \\
16,475 \\
16,626\end{array}$ & $\begin{array}{l}3,419 \\
3,461 \\
3,494 \\
3,544\end{array}$ \\
\hline $\begin{array}{l}\text { Females } \\
1981 \\
1986 \\
1991 \\
1996\end{array}$ & $\begin{array}{l}24,026 \\
24,239 \\
24,584 \\
24,890\end{array}$ & $\begin{array}{l}292 \\
301 \\
324 \\
293\end{array}$ & $\begin{array}{l}1,088 \\
1,161 \\
1,253 \\
1,229\end{array}$ & $\begin{array}{l}3,248 \\
2,859 \\
2,873 \\
3,056\end{array}$ & $\begin{array}{l}3,650 \\
3,761 \\
3,333 \\
2,961\end{array}$ & $\begin{array}{l}3,327 \\
3,325 \\
3,739 \\
3,849\end{array}$ & $\begin{array}{l}2,807 \\
3,229 \\
3,322 \\
3,336\end{array}$ & $\begin{array}{l}4,009 \\
3,850 \\
3,964 \\
4,432\end{array}$ & $\begin{array}{l}1,295 \\
1,335 \\
1,239 \\
1,177\end{array}$ & $\begin{array}{l}2,445 \\
2,346 \\
2,323 \\
2,286\end{array}$ & $\begin{array}{l}1,472 \\
1,604 \\
1,656 \\
1,628\end{array}$ & $\begin{array}{l}273 \\
326 \\
388 \\
430\end{array}$ & $\begin{array}{l}119 \\
140 \\
171 \\
214\end{array}$ & $\begin{array}{l}5,004 \\
4,672 \\
4,720 \\
4,876\end{array}$ & $\begin{array}{l}13,416 \\
13,815 \\
14,088 \\
14,281\end{array}$ & $\begin{array}{l}5,605 \\
5,752 \\
5,777 \\
5,734\end{array}$ \\
\hline $\begin{array}{l}2000 \\
2001 \\
2002 \\
2003\end{array}$ & $\begin{array}{l}25,203 \\
25,284 \\
25,362 \\
25,448\end{array}$ & $\begin{array}{l}281 \\
273 \\
273 \\
280\end{array}$ & $\begin{array}{l}1,174 \\
1,154 \\
1,131 \\
1,112\end{array}$ & $\begin{array}{l}3,109 \\
3,102 \\
3,095 \\
3,073\end{array}$ & $\begin{array}{l}2,928 \\
2,979 \\
3,026 \\
3,095\end{array}$ & $\begin{array}{l}3,667 \\
3,591 \\
3,503 \\
3,433\end{array}$ & $\begin{array}{l}3,653 \\
3,726 \\
3,795 \\
3,838\end{array}$ & $\begin{array}{l}4,637 \\
4,702 \\
4,767 \\
4,808\end{array}$ & $\begin{array}{l}1,227 \\
1,219 \\
1,223 \\
1,248\end{array}$ & $\begin{array}{l}2,190 \\
2,185 \\
2,187 \\
2,197\end{array}$ & $\begin{array}{l}1,649 \\
1,661 \\
1,676 \\
1,696\end{array}$ & $\begin{array}{l}448 \\
444 \\
433 \\
410\end{array}$ & $\begin{array}{l}240 \\
248 \\
254 \\
260\end{array}$ & $\begin{array}{l}4,867 \\
4,834 \\
4,808 \\
4,784\end{array}$ & $\begin{array}{l}14,582 \\
14,694 \\
14,782 \\
14,854\end{array}$ & $\begin{array}{l}5,755 \\
5,757 \\
5,772 \\
5,809\end{array}$ \\
\hline $\begin{array}{l}2004 \\
2005 \\
2006 \\
2007\end{array}$ & $\begin{array}{l}25,548 \\
25,708 \\
25,837 \\
25,978\end{array}$ & $\begin{array}{l}291 \\
296 \\
303 \\
312\end{array}$ & $\begin{array}{l}1,103 \\
1,117 \\
1,139 \\
1,170\end{array}$ & $\begin{array}{l}3,043 \\
3,001 \\
2,952 \\
2,910\end{array}$ & $\begin{array}{l}3,153 \\
3,218 \\
3,264 \\
3,306\end{array}$ & $\begin{array}{l}3,380 \\
3,378 \\
3,355 \\
3,324\end{array}$ & $\begin{array}{l}3,881 \\
3,905 \\
3,918 \\
3,917\end{array}$ & $\begin{array}{l}4,843 \\
4,885 \\
4,938 \\
4,937\end{array}$ & $\begin{array}{l}1,280 \\
1,319 \\
1,377 \\
1,485\end{array}$ & $\begin{array}{l}2,203 \\
2,206 \\
2,190 \\
2,198\end{array}$ & $\begin{array}{l}1,703 \\
1,686 \\
1,670 \\
1,660\end{array}$ & $\begin{array}{l}403 \\
428 \\
461 \\
488\end{array}$ & $\begin{array}{l}264 \\
269 \\
270 \\
270\end{array}$ & $\begin{array}{l}4,753 \\
4,733 \\
4,717 \\
4,711\end{array}$ & $\begin{array}{l}14,940 \\
15,066 \\
15,152 \\
15,166\end{array}$ & $\begin{array}{l}5,854 \\
5,908 \\
5,968 \\
6,100\end{array}$ \\
\hline $\begin{array}{l}\text { Wales } \\
\text { Persons } \\
1981 \\
1986 \\
1991 \\
1996\end{array}$ & $\begin{array}{l}2,813 \\
2,811 \\
2,873 \\
2,891\end{array}$ & $\begin{array}{l}36 \\
37 \\
38 \\
34\end{array}$ & $\begin{array}{l}136 \\
143 \\
153 \\
146\end{array}$ & $\begin{array}{l}407 \\
357 \\
363 \\
381\end{array}$ & $\begin{array}{l}434 \\
438 \\
393 \\
352\end{array}$ & $\begin{array}{l}383 \\
369 \\
402 \\
409\end{array}$ & $\begin{array}{l}333 \\
378 \\
389 \\
379\end{array}$ & $\begin{array}{l}485 \\
464 \\
486 \\
541\end{array}$ & $\begin{array}{l}158 \\
166 \\
154 \\
147\end{array}$ & $\begin{array}{l}272 \\
271 \\
284 \\
279\end{array}$ & $\begin{array}{l}139 \\
154 \\
164 \\
170\end{array}$ & $\begin{array}{l}21 \\
26 \\
32 \\
37\end{array}$ & $\begin{array}{r}8 \\
10 \\
13 \\
17\end{array}$ & $\begin{array}{l}626 \\
578 \\
589 \\
598\end{array}$ & $\begin{array}{l}1,663 \\
1,686 \\
1,711 \\
1,714\end{array}$ & $\begin{array}{l}525 \\
547 \\
573 \\
578\end{array}$ \\
\hline $\begin{array}{l}2000 \\
2001 \\
2002 \\
2003\end{array}$ & $\begin{array}{l}2,907 \\
2,910 \\
2,920 \\
2,931\end{array}$ & $\begin{array}{l}32 \\
32 \\
30 \\
31\end{array}$ & $\begin{array}{l}138 \\
136 \\
132 \\
129\end{array}$ & $\begin{array}{l}383 \\
382 \\
380 \\
377\end{array}$ & $\begin{array}{l}352 \\
356 \\
365 \\
376\end{array}$ & $\begin{array}{l}378 \\
365 \\
354 \\
345\end{array}$ & $\begin{array}{l}403 \\
409 \\
414 \\
417\end{array}$ & $\begin{array}{l}565 \\
572 \\
578 \\
582\end{array}$ & $\begin{array}{l}152 \\
154 \\
156 \\
161\end{array}$ & $\begin{array}{l}265 \\
264 \\
265 \\
268\end{array}$ & $\begin{array}{l}180 \\
183 \\
185 \\
187\end{array}$ & $\begin{array}{l}39 \\
39 \\
39 \\
38\end{array}$ & $\begin{array}{l}19 \\
20 \\
20 \\
21\end{array}$ & $\begin{array}{l}591 \\
587 \\
582 \\
577\end{array}$ & $\begin{array}{l}1,734 \\
1,739 \\
1,749 \\
1,759\end{array}$ & $\begin{array}{l}581 \\
584 \\
589 \\
595\end{array}$ \\
\hline $\begin{array}{l}2004 \\
2005 \\
2006 \\
2007\end{array}$ & $\begin{array}{l}2,946 \\
2,954 \\
2,966 \\
2,980\end{array}$ & $\begin{array}{l}32 \\
32 \\
33 \\
34\end{array}$ & $\begin{array}{l}127 \\
126 \\
127 \\
130\end{array}$ & $\begin{array}{l}373 \\
367 \\
361 \\
353\end{array}$ & $\begin{array}{l}385 \\
390 \\
399 \\
407\end{array}$ & $\begin{array}{l}339 \\
335 \\
332 \\
330\end{array}$ & $\begin{array}{l}421 \\
421 \\
421 \\
418\end{array}$ & $\begin{array}{l}586 \\
589 \\
592 \\
590\end{array}$ & $\begin{array}{l}166 \\
171 \\
177 \\
188\end{array}$ & $\begin{array}{l}270 \\
271 \\
273 \\
276\end{array}$ & $\begin{array}{l}188 \\
186 \\
186 \\
185\end{array}$ & $\begin{array}{l}39 \\
42 \\
45 \\
48\end{array}$ & $\begin{array}{l}21 \\
21 \\
22 \\
22\end{array}$ & $\begin{array}{l}572 \\
566 \\
561 \\
557\end{array}$ & $\begin{array}{l}1,773 \\
1,780 \\
1,790 \\
1,797\end{array}$ & $\begin{array}{l}602 \\
608 \\
615 \\
627\end{array}$ \\
\hline $\begin{array}{l}\text { Males } \\
1981 \\
1986 \\
1991 \\
1996\end{array}$ & $\begin{array}{l}1,365 \\
1,362 \\
1,391 \\
1,401\end{array}$ & $\begin{array}{l}18 \\
19 \\
20 \\
17\end{array}$ & $\begin{array}{l}70 \\
73 \\
78 \\
74\end{array}$ & $\begin{array}{l}209 \\
184 \\
186 \\
195\end{array}$ & $\begin{array}{l}221 \\
221 \\
199 \\
179\end{array}$ & $\begin{array}{l}193 \\
186 \\
199 \\
203\end{array}$ & $\begin{array}{l}168 \\
190 \\
194 \\
187\end{array}$ & $\begin{array}{l}240 \\
231 \\
242 \\
269\end{array}$ & $\begin{array}{l}73 \\
79 \\
74 \\
72\end{array}$ & $\begin{array}{l}118 \\
119 \\
128 \\
128\end{array}$ & $\begin{array}{l}48 \\
54 \\
60 \\
64\end{array}$ & $\begin{array}{r}5 \\
7 \\
8 \\
10\end{array}$ & $\begin{array}{l}2 \\
2 \\
2 \\
3\end{array}$ & $\begin{array}{l}321 \\
297 \\
302 \\
306\end{array}$ & $\begin{array}{l}871 \\
885 \\
891 \\
890\end{array}$ & $\begin{array}{l}173 \\
181 \\
198 \\
206\end{array}$ \\
\hline $\begin{array}{l}2000 \\
2001 \\
2002 \\
2003\end{array}$ & $\begin{array}{l}1,408 \\
1,409 \\
1,414 \\
1,423\end{array}$ & $\begin{array}{l}16 \\
16 \\
16 \\
16\end{array}$ & $\begin{array}{l}71 \\
69 \\
68 \\
66\end{array}$ & $\begin{array}{l}196 \\
196 \\
195 \\
194\end{array}$ & $\begin{array}{l}177 \\
179 \\
184 \\
190\end{array}$ & $\begin{array}{l}185 \\
178 \\
172 \\
168\end{array}$ & $\begin{array}{l}198 \\
200 \\
202 \\
204\end{array}$ & $\begin{array}{l}280 \\
283 \\
285 \\
287\end{array}$ & $\begin{array}{l}75 \\
75 \\
77 \\
79\end{array}$ & $\begin{array}{l}124 \\
124 \\
125 \\
127\end{array}$ & $\begin{array}{l}71 \\
73 \\
74 \\
75\end{array}$ & $\begin{array}{l}12 \\
12 \\
12 \\
11\end{array}$ & $\begin{array}{l}4 \\
4 \\
5 \\
5\end{array}$ & $\begin{array}{l}303 \\
301 \\
299 \\
296\end{array}$ & $\begin{array}{l}895 \\
895 \\
900 \\
908\end{array}$ & $\begin{array}{l}210 \\
212 \\
215 \\
218\end{array}$ \\
\hline $\begin{array}{l}2004 \\
2005 \\
2006 \\
2007\end{array}$ & $\begin{array}{l}1,432 \\
1,439 \\
1,445 \\
1,454\end{array}$ & $\begin{array}{l}16 \\
17 \\
17 \\
17\end{array}$ & $\begin{array}{l}65 \\
65 \\
65 \\
67\end{array}$ & $\begin{array}{l}192 \\
189 \\
185 \\
181\end{array}$ & $\begin{array}{l}196 \\
200 \\
204 \\
209\end{array}$ & $\begin{array}{l}166 \\
166 \\
164 \\
164\end{array}$ & $\begin{array}{l}205 \\
205 \\
205 \\
203\end{array}$ & $\begin{array}{l}288 \\
290 \\
291 \\
289\end{array}$ & $\begin{array}{l}82 \\
84 \\
87 \\
93\end{array}$ & $\begin{array}{l}128 \\
129 \\
130 \\
132\end{array}$ & $\begin{array}{l}76 \\
77 \\
77 \\
78\end{array}$ & $\begin{array}{l}12 \\
13 \\
15 \\
16\end{array}$ & $\begin{array}{l}5 \\
5 \\
5 \\
6\end{array}$ & $\begin{array}{l}294 \\
291 \\
288 \\
286\end{array}$ & $\begin{array}{l}917 \\
924 \\
929 \\
937\end{array}$ & $\begin{array}{l}221 \\
224 \\
227 \\
231\end{array}$ \\
\hline $\begin{array}{l}\text { Females } \\
1981 \\
1986 \\
1991 \\
1996\end{array}$ & $\begin{array}{l}1,448 \\
1,449 \\
1,482 \\
1,490\end{array}$ & $\begin{array}{l}18 \\
18 \\
19 \\
16\end{array}$ & $\begin{array}{l}66 \\
70 \\
75 \\
71\end{array}$ & $\begin{array}{l}199 \\
173 \\
177 \\
186\end{array}$ & $\begin{array}{l}213 \\
217 \\
194 \\
173\end{array}$ & $\begin{array}{l}190 \\
184 \\
203 \\
206\end{array}$ & $\begin{array}{l}165 \\
188 \\
195 \\
192\end{array}$ & $\begin{array}{l}246 \\
233 \\
244 \\
272\end{array}$ & $\begin{array}{l}85 \\
87 \\
80 \\
75\end{array}$ & $\begin{array}{l}154 \\
152 \\
156 \\
151\end{array}$ & $\begin{array}{r}91 \\
100 \\
104 \\
106\end{array}$ & $\begin{array}{l}16 \\
20 \\
24 \\
27\end{array}$ & $\begin{array}{r}6 \\
8 \\
10 \\
13\end{array}$ & $\begin{array}{l}305 \\
282 \\
288 \\
293\end{array}$ & $\begin{array}{l}791 \\
801 \\
820 \\
825\end{array}$ & $\begin{array}{l}352 \\
366 \\
375 \\
373\end{array}$ \\
\hline $\begin{array}{l}2000 \\
2001 \\
2002 \\
2003\end{array}$ & $\begin{array}{l}1,499 \\
1,502 \\
1,506 \\
1,508\end{array}$ & $\begin{array}{l}15 \\
15 \\
15 \\
15\end{array}$ & $\begin{array}{l}67 \\
66 \\
65 \\
63\end{array}$ & $\begin{array}{l}186 \\
186 \\
185 \\
183\end{array}$ & $\begin{array}{l}175 \\
177 \\
181 \\
185\end{array}$ & $\begin{array}{l}192 \\
187 \\
182 \\
176\end{array}$ & $\begin{array}{l}206 \\
209 \\
212 \\
214\end{array}$ & $\begin{array}{l}285 \\
289 \\
293 \\
295\end{array}$ & $\begin{array}{l}77 \\
78 \\
80 \\
82\end{array}$ & $\begin{array}{l}142 \\
141 \\
140 \\
141\end{array}$ & $\begin{array}{l}109 \\
110 \\
111 \\
112\end{array}$ & $\begin{array}{l}28 \\
27 \\
27 \\
27\end{array}$ & $\begin{array}{l}15 \\
15 \\
16 \\
16\end{array}$ & $\begin{array}{l}288 \\
286 \\
283 \\
280\end{array}$ & $\begin{array}{l}840 \\
844 \\
849 \\
851\end{array}$ & $\begin{array}{l}371 \\
372 \\
374 \\
377\end{array}$ \\
\hline $\begin{array}{l}2004 \\
2005 \\
2006 \\
2007\end{array}$ & $\begin{array}{l}1,514 \\
1,515 \\
1,521 \\
1,526\end{array}$ & $\begin{array}{l}15 \\
16 \\
16 \\
16\end{array}$ & $\begin{array}{l}62 \\
61 \\
62 \\
63\end{array}$ & $\begin{array}{l}182 \\
179 \\
176 \\
172\end{array}$ & $\begin{array}{l}189 \\
191 \\
195 \\
198 \\
\end{array}$ & $\begin{array}{l}172 \\
170 \\
168 \\
166\end{array}$ & $\begin{array}{l}216 \\
216 \\
216 \\
215\end{array}$ & $\begin{array}{l}298 \\
299 \\
301 \\
300\end{array}$ & $\begin{array}{l}84 \\
87 \\
90 \\
96\end{array}$ & $\begin{array}{l}142 \\
142 \\
143 \\
144\end{array}$ & $\begin{array}{l}112 \\
110 \\
108 \\
107\end{array}$ & $\begin{array}{l}26 \\
28 \\
30 \\
32 \\
\end{array}$ & $\begin{array}{l}16 \\
16 \\
16 \\
16\end{array}$ & $\begin{array}{l}278 \\
275 \\
273 \\
271\end{array}$ & $\begin{array}{l}856 \\
856 \\
861 \\
860\end{array}$ & $\begin{array}{l}380 \\
383 \\
387 \\
395\end{array}$ \\
\hline
\end{tabular}

See notes on first page of table. 


\begin{tabular}{|c|c|c|c|c|c|c|c|c|c|c|c|c|c|c|c|c|}
\hline $\begin{array}{l}\text { Table } 1.4 \\
\text { continued }\end{array}$ & \multicolumn{16}{|c|}{ Population: age and sex } \\
\hline \multicolumn{15}{|c|}{ Constituent countries of the United Kingdom } & \multicolumn{2}{|c|}{ Numbers (thousands } \\
\hline & & \multicolumn{15}{|c|}{ Age group } \\
\hline Mid-year & All ages & Under 1 & $1-4$ & $5-14$ & $15-24$ & $25-34$ & $35-44$ & $45-59$ & $60-64$ & $65-74$ & $75-84$ & $85-89$ & $\begin{array}{l}90 \text { and } \\
\text { over }\end{array}$ & $\begin{array}{l}\text { Under } \\
16\end{array}$ & \begin{tabular}{|c|}
$16-$ \\
$64 \mathrm{M} / 59 \mathrm{~F}^{1}$
\end{tabular} & $\begin{array}{l}65 \mathrm{M} / 60 \mathrm{~F}^{1} \\
\text { and over }\end{array}$ \\
\hline $\begin{array}{l}\text { Scotland } \\
\text { Persons } \\
1981 \\
1986 \\
1991 \\
1996\end{array}$ & $\begin{array}{l}5,180 \\
5,112 \\
5,083 \\
5,092\end{array}$ & $\begin{array}{l}69 \\
66 \\
66 \\
59\end{array}$ & $\begin{array}{l}249 \\
257 \\
258 \\
252\end{array}$ & $\begin{array}{l}780 \\
656 \\
634 \\
643\end{array}$ & $\begin{array}{l}875 \\
863 \\
746 \\
651\end{array}$ & $\begin{array}{l}724 \\
739 \\
795 \\
798\end{array}$ & $\begin{array}{l}603 \\
665 \\
696 \\
722\end{array}$ & $\begin{array}{l}880 \\
849 \\
853 \\
925\end{array}$ & $\begin{array}{l}260 \\
273 \\
265 \\
259\end{array}$ & $\begin{array}{l}460 \\
435 \\
441 \\
448\end{array}$ & $\begin{array}{l}232 \\
252 \\
259 \\
256\end{array}$ & $\begin{array}{l}35 \\
42 \\
51 \\
57\end{array}$ & $\begin{array}{l}14 \\
15 \\
19 \\
24\end{array}$ & $\begin{array}{l}1,188 \\
1,061 \\
1,021 \\
1,019\end{array}$ & $\begin{array}{l}3,110 \\
3,161 \\
3,151 \\
3,151\end{array}$ & $\begin{array}{l}882 \\
890 \\
912 \\
922\end{array}$ \\
\hline $\begin{array}{l}2000 \\
2001 \\
2002 \\
2003\end{array}$ & $\begin{array}{l}5,063 \\
5,064 \\
5,055 \\
5,057\end{array}$ & $\begin{array}{l}53 \\
52 \\
51 \\
52\end{array}$ & $\begin{array}{l}230 \\
224 \\
217 \\
212\end{array}$ & $\begin{array}{l}636 \\
629 \\
622 \\
614\end{array}$ & $\begin{array}{l}628 \\
633 \\
639 \\
648\end{array}$ & $\begin{array}{l}717 \\
696 \\
669 \\
648\end{array}$ & $\begin{array}{l}774 \\
782 \\
788 \\
793\end{array}$ & $\begin{array}{r}962 \\
979 \\
993 \\
1,008\end{array}$ & $\begin{array}{l}263 \\
262 \\
262 \\
265\end{array}$ & $\begin{array}{l}445 \\
447 \\
449 \\
452\end{array}$ & $\begin{array}{l}267 \\
272 \\
276 \\
281\end{array}$ & $\begin{array}{l}59 \\
59 \\
58 \\
55\end{array}$ & $\begin{array}{l}28 \\
29 \\
30 \\
31\end{array}$ & $\begin{array}{l}985 \\
970 \\
955 \\
943\end{array}$ & $\begin{array}{l}3,141 \\
3,150 \\
3,150 \\
3,156\end{array}$ & $\begin{array}{l}937 \\
944 \\
950 \\
958\end{array}$ \\
\hline $\begin{array}{l}2004 \\
2005 \\
2006 \\
2007\end{array}$ & $\begin{array}{l}5,078 \\
5,095 \\
5,117 \\
5,144\end{array}$ & $\begin{array}{l}54 \\
54 \\
55 \\
57\end{array}$ & $\begin{array}{l}210 \\
211 \\
213 \\
218\end{array}$ & $\begin{array}{l}609 \\
600 \\
588 \\
576\end{array}$ & $\begin{array}{l}653 \\
659 \\
668 \\
676\end{array}$ & $\begin{array}{l}635 \\
629 \\
627 \\
629\end{array}$ & $\begin{array}{l}796 \\
794 \\
790 \\
781\end{array}$ & $\begin{array}{l}1,025 \\
1,042 \\
1,058 \\
1,060\end{array}$ & $\begin{array}{l}270 \\
273 \\
280 \\
301\end{array}$ & $\begin{array}{l}455 \\
457 \\
456 \\
457\end{array}$ & $\begin{array}{l}286 \\
286 \\
287 \\
290\end{array}$ & $\begin{array}{l}54 \\
59 \\
63 \\
66\end{array}$ & $\begin{array}{l}31 \\
32 \\
32 \\
32\end{array}$ & $\begin{array}{l}935 \\
929 \\
922 \\
917\end{array}$ & $\begin{array}{l}3,175 \\
3,191 \\
3,213 \\
3,227\end{array}$ & $\begin{array}{r}968 \\
975 \\
983 \\
1,001\end{array}$ \\
\hline $\begin{array}{l}\text { Males } \\
1981 \\
1986 \\
1991 \\
1996\end{array}$ & $\begin{array}{l}2,495 \\
2,462 \\
2,445 \\
2,447\end{array}$ & $\begin{array}{l}35 \\
34 \\
34 \\
30\end{array}$ & $\begin{array}{l}128 \\
131 \\
132 \\
128\end{array}$ & $\begin{array}{l}400 \\
336 \\
324 \\
328\end{array}$ & $\begin{array}{l}445 \\
438 \\
377 \\
327\end{array}$ & $\begin{array}{l}364 \\
371 \\
394 \\
392\end{array}$ & $\begin{array}{l}298 \\
331 \\
345 \\
355\end{array}$ & $\begin{array}{l}424 \\
410 \\
415 \\
454\end{array}$ & $\begin{array}{l}118 \\
127 \\
124 \\
122\end{array}$ & $\begin{array}{l}194 \\
184 \\
192 \\
198\end{array}$ & $\begin{array}{l}77 \\
86 \\
91 \\
93\end{array}$ & $\begin{array}{r}8 \\
10 \\
13 \\
15\end{array}$ & $\begin{array}{l}3 \\
3 \\
3 \\
5\end{array}$ & $\begin{array}{l}610 \\
543 \\
522 \\
521\end{array}$ & $\begin{array}{l}1,603 \\
1,636 \\
1,623 \\
1,616\end{array}$ & $\begin{array}{l}282 \\
283 \\
299 \\
310\end{array}$ \\
\hline $\begin{array}{l}2000 \\
2001 \\
2002 \\
2003\end{array}$ & $\begin{array}{l}2,432 \\
2,434 \\
2,432 \\
2,435\end{array}$ & $\begin{array}{l}28 \\
26 \\
26 \\
26\end{array}$ & $\begin{array}{l}118 \\
115 \\
111 \\
108\end{array}$ & $\begin{array}{l}326 \\
322 \\
319 \\
314\end{array}$ & $\begin{array}{l}315 \\
319 \\
324 \\
329\end{array}$ & $\begin{array}{l}347 \\
337 \\
325 \\
315\end{array}$ & $\begin{array}{l}377 \\
379 \\
382 \\
383\end{array}$ & $\begin{array}{l}474 \\
483 \\
490 \\
496\end{array}$ & $\begin{array}{l}125 \\
125 \\
125 \\
126\end{array}$ & $\begin{array}{l}199 \\
200 \\
202 \\
204\end{array}$ & $\begin{array}{l}100 \\
103 \\
106 \\
108\end{array}$ & $\begin{array}{l}17 \\
17 \\
17 \\
16\end{array}$ & $\begin{array}{l}6 \\
6 \\
7 \\
7\end{array}$ & $\begin{array}{l}505 \\
497 \\
489 \\
483\end{array}$ & $\begin{array}{l}1,606 \\
1,610 \\
1,612 \\
1,616\end{array}$ & $\begin{array}{l}322 \\
327 \\
331 \\
336\end{array}$ \\
\hline $\begin{array}{l}2004 \\
2005 \\
2006 \\
2007\end{array}$ & $\begin{array}{r}2,446 \\
2,456 \\
2,469 \\
2,486\end{array}$ & $\begin{array}{l}28 \\
28 \\
28 \\
29\end{array}$ & $\begin{array}{l}107 \\
107 \\
109 \\
112\end{array}$ & $\begin{array}{l}312 \\
307 \\
301 \\
295\end{array}$ & $\begin{array}{l}332 \\
335 \\
340 \\
345\end{array}$ & $\begin{array}{l}310 \\
309 \\
310 \\
313\end{array}$ & $\begin{array}{l}384 \\
382 \\
380 \\
375\end{array}$ & $\begin{array}{l}503 \\
511 \\
517 \\
517\end{array}$ & $\begin{array}{l}129 \\
131 \\
135 \\
146\end{array}$ & $\begin{array}{l}207 \\
208 \\
208 \\
210\end{array}$ & $\begin{array}{l}111 \\
112 \\
113 \\
116\end{array}$ & $\begin{array}{l}16 \\
18 \\
20 \\
21\end{array}$ & $\begin{array}{l}7 \\
7 \\
8 \\
8\end{array}$ & $\begin{array}{l}479 \\
476 \\
472 \\
469\end{array}$ & $\begin{array}{l}1,627 \\
1,6635 \\
1,649 \\
1,662\end{array}$ & $\begin{array}{l}341 \\
345 \\
349 \\
354\end{array}$ \\
\hline $\begin{array}{l}\text { Females } \\
1981 \\
1986 \\
1991 \\
1996\end{array}$ & $\begin{array}{l}2,685 \\
2,649 \\
2,639 \\
2,645\end{array}$ & $\begin{array}{l}33 \\
32 \\
32 \\
28\end{array}$ & $\begin{array}{l}121 \\
126 \\
126 \\
123\end{array}$ & $\begin{array}{l}380 \\
320 \\
309 \\
315\end{array}$ & $\begin{array}{l}430 \\
424 \\
369 \\
324\end{array}$ & $\begin{array}{l}359 \\
368 \\
402 \\
406\end{array}$ & $\begin{array}{l}305 \\
334 \\
351 \\
367\end{array}$ & $\begin{array}{l}456 \\
439 \\
437 \\
470\end{array}$ & $\begin{array}{l}142 \\
146 \\
141 \\
137\end{array}$ & $\begin{array}{l}265 \\
250 \\
249 \\
250\end{array}$ & $\begin{array}{l}155 \\
166 \\
168 \\
164\end{array}$ & $\begin{array}{l}27 \\
32 \\
38 \\
42\end{array}$ & $\begin{array}{l}11 \\
12 \\
16 \\
20\end{array}$ & $\begin{array}{l}579 \\
518 \\
499 \\
498\end{array}$ & $\begin{array}{l}1,506 \\
1,525 \\
1,528 \\
1,535\end{array}$ & $\begin{array}{l}600 \\
606 \\
612 \\
612\end{array}$ \\
\hline $\begin{array}{l}2000 \\
2001 \\
2002 \\
2003\end{array}$ & $\begin{array}{l}2,631 \\
2,630 \\
2,623 \\
2,623\end{array}$ & $\begin{array}{l}26 \\
26 \\
25 \\
25\end{array}$ & $\begin{array}{l}112 \\
109 \\
106 \\
104\end{array}$ & $\begin{array}{l}310 \\
307 \\
303 \\
300\end{array}$ & $\begin{array}{l}313 \\
314 \\
315 \\
318\end{array}$ & $\begin{array}{l}369 \\
359 \\
344 \\
332\end{array}$ & $\begin{array}{l}397 \\
403 \\
406 \\
410\end{array}$ & $\begin{array}{l}488 \\
496 \\
504 \\
512\end{array}$ & $\begin{array}{l}138 \\
137 \\
137 \\
139\end{array}$ & $\begin{array}{l}246 \\
246 \\
247 \\
248\end{array}$ & $\begin{array}{l}166 \\
169 \\
171 \\
173\end{array}$ & $\begin{array}{l}43 \\
43 \\
41 \\
39\end{array}$ & $\begin{array}{l}22 \\
23 \\
23 \\
24\end{array}$ & $\begin{array}{l}480 \\
473 \\
466 \\
460\end{array}$ & $\begin{array}{l}1,535 \\
1,540 \\
1,538 \\
1,540\end{array}$ & $\begin{array}{l}616 \\
617 \\
619 \\
622\end{array}$ \\
\hline $\begin{array}{l}2004 \\
2005 \\
2006 \\
2007\end{array}$ & $\begin{array}{l}2,632 \\
2,639 \\
2,647 \\
2,659\end{array}$ & $\begin{array}{l}26 \\
26 \\
27 \\
28\end{array}$ & $\begin{array}{l}103 \\
103 \\
104 \\
106\end{array}$ & $\begin{array}{l}297 \\
293 \\
287 \\
281\end{array}$ & $\begin{array}{l}321 \\
324 \\
328 \\
332\end{array}$ & $\begin{array}{l}325 \\
320 \\
317 \\
316\end{array}$ & $\begin{array}{l}412 \\
411 \\
410 \\
406\end{array}$ & $\begin{array}{l}521 \\
531 \\
541 \\
542\end{array}$ & $\begin{array}{l}141 \\
142 \\
145 \\
155\end{array}$ & $\begin{array}{l}248 \\
249 \\
247 \\
247\end{array}$ & $\begin{array}{l}175 \\
174 \\
174 \\
174\end{array}$ & $\begin{array}{l}38 \\
41 \\
43 \\
45\end{array}$ & $\begin{array}{l}24 \\
25 \\
25 \\
24\end{array}$ & $\begin{array}{l}457 \\
453 \\
450 \\
448\end{array}$ & $\begin{array}{l}1,549 \\
1,556 \\
1,564 \\
1,564\end{array}$ & $\begin{array}{l}627 \\
630 \\
634 \\
646\end{array}$ \\
\hline $\begin{array}{l}\text { Northern Irela } \\
\text { Persons } \\
1981 \\
1986 \\
1991 \\
1996\end{array}$ & $\begin{array}{l}1,543 \\
1,574 \\
1,607 \\
1,662\end{array}$ & $\begin{array}{l}27 \\
28 \\
26 \\
24\end{array}$ & $\begin{array}{r}106 \\
107 \\
106 \\
99\end{array}$ & $\begin{array}{l}282 \\
261 \\
260 \\
266\end{array}$ & $\begin{array}{l}271 \\
277 \\
256 \\
244\end{array}$ & $\begin{array}{l}200 \\
217 \\
240 \\
257\end{array}$ & $\begin{array}{l}175 \\
190 \\
200 \\
220\end{array}$ & $\begin{array}{l}227 \\
227 \\
241 \\
266\end{array}$ & $\begin{array}{l}68 \\
71 \\
70 \\
70\end{array}$ & $\begin{array}{l}116 \\
115 \\
121 \\
123\end{array}$ & $\begin{array}{l}57 \\
64 \\
69 \\
72\end{array}$ & $\begin{array}{l}10 \\
14 \\
15\end{array}$ & $\begin{array}{l}. . \\
\ddot{6} \\
7\end{array}$ & $\begin{array}{l}444 \\
423 \\
417 \\
415\end{array}$ & $\begin{array}{l}874 \\
917 \\
945 \\
993\end{array}$ & $\begin{array}{l}224 \\
234 \\
246 \\
253\end{array}$ \\
\hline $\begin{array}{l}2000 \\
2001 \\
2002 \\
2003\end{array}$ & $\begin{array}{l}1,683 \\
1,689 \\
1,697 \\
1,703\end{array}$ & $\begin{array}{l}22 \\
22 \\
22 \\
21\end{array}$ & $\begin{array}{l}95 \\
93 \\
91 \\
89\end{array}$ & $\begin{array}{l}259 \\
255 \\
253 \\
251\end{array}$ & $\begin{array}{l}237 \\
240 \\
243 \\
246\end{array}$ & $\begin{array}{l}247 \\
243 \\
238 \\
233\end{array}$ & $\begin{array}{l}243 \\
248 \\
251 \\
254\end{array}$ & $\begin{array}{l}284 \\
290 \\
296 \\
301\end{array}$ & $\begin{array}{l}73 \\
74 \\
75 \\
78\end{array}$ & $\begin{array}{l}123 \\
123 \\
125 \\
126\end{array}$ & $\begin{array}{l}75 \\
77 \\
79 \\
81\end{array}$ & $\begin{array}{l}16 \\
16 \\
16 \\
16\end{array}$ & $\begin{array}{l}7 \\
7 \\
7 \\
8\end{array}$ & $\begin{array}{l}403 \\
397 \\
393 \\
388\end{array}$ & $\begin{array}{l}1,020 \\
1,030 \\
1,037 \\
1,044\end{array}$ & $\begin{array}{l}259 \\
262 \\
266 \\
271\end{array}$ \\
\hline $\begin{array}{l}2004 \\
2005 \\
2006 \\
2007\end{array}$ & $\begin{array}{l}1,710 \\
1,724 \\
1,742 \\
1,759\end{array}$ & $\begin{array}{l}22 \\
23 \\
23 \\
24\end{array}$ & $\begin{array}{l}87 \\
88 \\
89 \\
91\end{array}$ & $\begin{array}{l}248 \\
245 \\
242 \\
239\end{array}$ & $\begin{array}{l}250 \\
253 \\
258 \\
260\end{array}$ & $\begin{array}{l}229 \\
228 \\
229 \\
231\end{array}$ & $\begin{array}{l}256 \\
257 \\
259 \\
259\end{array}$ & $\begin{array}{l}305 \\
310 \\
316 \\
321\end{array}$ & $\begin{array}{l}81 \\
84 \\
87 \\
90\end{array}$ & $\begin{array}{l}127 \\
128 \\
130 \\
132\end{array}$ & $\begin{array}{l}82 \\
83 \\
83 \\
84\end{array}$ & $\begin{array}{l}16 \\
17 \\
18 \\
19\end{array}$ & $\begin{array}{l}8 \\
8 \\
8 \\
8\end{array}$ & $\begin{array}{l}383 \\
381 \\
380 \\
380\end{array}$ & $\begin{array}{l}1,052 \\
1,064 \\
1,077 \\
1,089\end{array}$ & $\begin{array}{l}275 \\
280 \\
284 \\
290\end{array}$ \\
\hline $\begin{array}{l}\text { Males } \\
1981 \\
1986 \\
1991 \\
1996\end{array}$ & $\begin{array}{l}757 \\
768 \\
783 \\
810\end{array}$ & $\begin{array}{l}14 \\
14 \\
13 \\
12\end{array}$ & $\begin{array}{l}54 \\
55 \\
54 \\
51\end{array}$ & $\begin{array}{l}145 \\
134 \\
133 \\
136\end{array}$ & $\begin{array}{l}140 \\
142 \\
131 \\
124\end{array}$ & $\begin{array}{l}102 \\
109 \\
119 \\
128\end{array}$ & $\begin{array}{r}87 \\
95 \\
100 \\
109\end{array}$ & $\begin{array}{l}109 \\
110 \\
118 \\
131\end{array}$ & $\begin{array}{l}32 \\
33 \\
32 \\
33\end{array}$ & $\begin{array}{l}50 \\
50 \\
53 \\
54\end{array}$ & $\begin{array}{l}21 \\
23 \\
26 \\
27\end{array}$ & $\begin{array}{l}\ddot{4} \\
4 \\
4\end{array}$ & $\begin{array}{l}\ddot{.} \\
\ddot{1} \\
1\end{array}$ & $\begin{array}{l}228 \\
217 \\
213 \\
212\end{array}$ & $\begin{array}{l}454 \\
474 \\
487 \\
511\end{array}$ & $\begin{array}{l}75 \\
77 \\
83 \\
87\end{array}$ \\
\hline $\begin{array}{l}2000 \\
2001 \\
2002 \\
2003\end{array}$ & $\begin{array}{l}820 \\
824 \\
829 \\
833\end{array}$ & $\begin{array}{l}11 \\
11 \\
11 \\
11\end{array}$ & $\begin{array}{l}49 \\
48 \\
47 \\
46\end{array}$ & $\begin{array}{l}133 \\
131 \\
130 \\
129\end{array}$ & $\begin{array}{l}120 \\
122 \\
124 \\
126\end{array}$ & $\begin{array}{l}122 \\
120 \\
117 \\
115\end{array}$ & $\begin{array}{l}119 \\
122 \\
123 \\
124\end{array}$ & $\begin{array}{l}141 \\
144 \\
147 \\
149\end{array}$ & $\begin{array}{l}35 \\
35 \\
36 \\
38\end{array}$ & $\begin{array}{l}55 \\
56 \\
56 \\
57\end{array}$ & $\begin{array}{l}29 \\
30 \\
31 \\
31\end{array}$ & $\begin{array}{l}5 \\
5 \\
5 \\
5\end{array}$ & $\begin{array}{l}2 \\
2 \\
2 \\
2\end{array}$ & $\begin{array}{l}207 \\
204 \\
202 \\
199\end{array}$ & $\begin{array}{l}524 \\
529 \\
534 \\
538\end{array}$ & $\begin{array}{l}90 \\
92 \\
94 \\
95\end{array}$ \\
\hline $\begin{array}{l}2004 \\
2005 \\
2006 \\
2007\end{array}$ & $\begin{array}{l}836 \\
844 \\
853 \\
862\end{array}$ & $\begin{array}{l}11 \\
12 \\
12 \\
13\end{array}$ & $\begin{array}{l}45 \\
45 \\
46 \\
47\end{array}$ & $\begin{array}{l}127 \\
126 \\
124 \\
123\end{array}$ & $\begin{array}{l}128 \\
130 \\
132 \\
134\end{array}$ & $\begin{array}{l}113 \\
113 \\
113 \\
114\end{array}$ & $\begin{array}{l}125 \\
126 \\
127 \\
127\end{array}$ & $\begin{array}{l}151 \\
153 \\
156 \\
158\end{array}$ & $\begin{array}{l}39 \\
41 \\
42 \\
44\end{array}$ & $\begin{array}{l}58 \\
59 \\
60 \\
61\end{array}$ & $\begin{array}{l}32 \\
32 \\
33 \\
33\end{array}$ & $\begin{array}{l}5 \\
5 \\
6 \\
6\end{array}$ & $\begin{array}{l}2 \\
2 \\
2 \\
2\end{array}$ & $\begin{array}{l}197 \\
196 \\
195 \\
195\end{array}$ & $\begin{array}{l}542 \\
550 \\
558 \\
564\end{array}$ & $\begin{array}{r}97 \\
99 \\
101 \\
103\end{array}$ \\
\hline $\begin{array}{l}\text { Females } \\
1981 \\
1986 \\
1991 \\
1996\end{array}$ & $\begin{array}{l}786 \\
805 \\
824 \\
851\end{array}$ & $\begin{array}{l}13 \\
13 \\
13 \\
11\end{array}$ & $\begin{array}{l}52 \\
52 \\
52 \\
49\end{array}$ & $\begin{array}{l}137 \\
127 \\
127 \\
130\end{array}$ & $\begin{array}{l}130 \\
135 \\
125 \\
120\end{array}$ & $\begin{array}{r}98 \\
107 \\
121 \\
129\end{array}$ & $\begin{array}{r}88 \\
96 \\
100 \\
110\end{array}$ & $\begin{array}{l}118 \\
118 \\
123 \\
135\end{array}$ & $\begin{array}{l}37 \\
38 \\
38 \\
37\end{array}$ & $\begin{array}{l}66 \\
65 \\
67 \\
69\end{array}$ & $\begin{array}{l}37 \\
41 \\
44 \\
45\end{array}$ & $\begin{array}{l}12 \\
10 \\
11\end{array}$ & $\begin{array}{l}\ddot{y} \\
\ddot{4} \\
6\end{array}$ & $\begin{array}{l}216 \\
206 \\
203 \\
203\end{array}$ & $\begin{array}{l}420 \\
442 \\
458 \\
482\end{array}$ & $\begin{array}{l}150 \\
157 \\
163 \\
167\end{array}$ \\
\hline $\begin{array}{l}2000 \\
2001 \\
2002 \\
2003\end{array}$ & $\begin{array}{l}862 \\
865 \\
868 \\
870\end{array}$ & $\begin{array}{l}11 \\
10 \\
11 \\
10\end{array}$ & $\begin{array}{l}46 \\
45 \\
44 \\
43\end{array}$ & $\begin{array}{l}126 \\
124 \\
123 \\
122\end{array}$ & $\begin{array}{l}118 \\
119 \\
119 \\
120\end{array}$ & $\begin{array}{l}125 \\
123 \\
120 \\
118\end{array}$ & $\begin{array}{l}124 \\
126 \\
128 \\
129\end{array}$ & $\begin{array}{l}143 \\
146 \\
149 \\
152\end{array}$ & $\begin{array}{l}38 \\
38 \\
39 \\
40\end{array}$ & $\begin{array}{l}68 \\
68 \\
68 \\
68\end{array}$ & $\begin{array}{l}46 \\
47 \\
48 \\
49\end{array}$ & $\begin{array}{l}11 \\
11 \\
11 \\
11\end{array}$ & $\begin{array}{l}6 \\
6 \\
6 \\
6\end{array}$ & $\begin{array}{l}196 \\
193 \\
191 \\
189\end{array}$ & $\begin{array}{l}497 \\
501 \\
504 \\
506\end{array}$ & $\begin{array}{l}169 \\
170 \\
173 \\
175\end{array}$ \\
\hline $\begin{array}{l}2004 \\
2005 \\
2006 \\
2007\end{array}$ & $\begin{array}{l}874 \\
880 \\
888 \\
897\end{array}$ & $\begin{array}{l}11 \\
11 \\
11 \\
12\end{array}$ & $\begin{array}{l}42 \\
43 \\
43 \\
44\end{array}$ & $\begin{array}{l}121 \\
119 \\
118 \\
116\end{array}$ & $\begin{array}{l}122 \\
123 \\
126 \\
127\end{array}$ & $\begin{array}{l}116 \\
115 \\
115 \\
117\end{array}$ & $\begin{array}{l}130 \\
131 \\
132 \\
132\end{array}$ & $\begin{array}{l}154 \\
157 \\
160 \\
163\end{array}$ & $\begin{array}{l}42 \\
43 \\
45 \\
46\end{array}$ & $\begin{array}{l}69 \\
69 \\
69 \\
70\end{array}$ & $\begin{array}{l}50 \\
50 \\
51 \\
51\end{array}$ & $\begin{array}{l}11 \\
11 \\
12 \\
13\end{array}$ & $\begin{array}{l}6 \\
6 \\
6 \\
6\end{array}$ & $\begin{array}{l}187 \\
186 \\
185 \\
185\end{array}$ & $\begin{array}{l}509 \\
514 \\
520 \\
526\end{array}$ & $\begin{array}{l}178 \\
181 \\
183 \\
187\end{array}$ \\
\hline
\end{tabular}

See notes on first page of table. 


\begin{tabular}{|c|c|c|c|c|c|c|c|c|c|c|c|}
\hline Table 1.5 & Populatic & ge, se & legal m & al status & & & & & & & \\
\hline England and Wales & & & & & & & & & & Num & thousands) \\
\hline & Total & & & Males & & & & & Females & & \\
\hline Mid-year & pop & Single & Married & Divorced & Widowed & Total & Single & Married & Divorced & Widowed & Total \\
\hline Aged & & & & & & & & & & & \\
\hline 16 and over & & & & & & & & & & & \\
\hline 1971 & 36,818 & 4,173 & 12,522 & 187 & 682 & 17,563 & 3,583 & 12,566 & 296 & 2,810 & 19,255 \\
\hline 1976 & 37,486 & 4,369 & 12,511 & 376 & 686 & 17,941 & 3,597 & 12,538 & 533 & 2,877 & 19,545 \\
\hline 1981 & 38,724 & 5,013 & 12,238 & 611 & 698 & 18,559 & 4,114 & 12,284 & 828 & 2,939 & 20,165 \\
\hline 1986 & 39,837 & 5,625 & 11,867 & 917 & 695 & 19,103 & 4,617 & 12,000 & 1,165 & 2,953 & 20,734 \\
\hline 1991 & 40,501 & 5,891 & 11,636 & 1,187 & 727 & 19,441 & 4,817 & 11,833 & 1,459 & 2,951 & 21,060 \\
\hline 1996 & 40,827 & 6,225 & 11,310 & 1,346 & 733 & 19,614 & 5,168 & 11,433 & 1,730 & 2,881 & 21,212 \\
\hline 1999 & 41,325 & 6,582 & 11,143 & 1,433 & 732 & 19,890 & 5,526 & 11,235 & 1,875 & 2,800 & 21,435 \\
\hline 2000 & 41,569 & 6,721 & 11,113 & 1,456 & 731 & 20,022 & 5,650 & 11,199 & 1,927 & 2,772 & 21,547 \\
\hline 2001 & 41,865 & 6,894 & 11,090 & 1,482 & 733 & 20,198 & 5,798 & 11,150 & 1,975 & 2,745 & 21,667 \\
\hline 2002 & 42,135 & 7,086 & 11,008 & 1,534 & 730 & 20,358 & 5,957 & 11,075 & 2,036 & 2,710 & 21,777 \\
\hline 2003 & 42,409 & 7,272 & 10,929 & 1,589 & 727 & 20,517 & 6,126 & 11,000 & 2,096 & 2,669 & 21,892 \\
\hline 2004 & 42,731 & 7,483 & 10,851 & 1,642 & 724 & 20,700 & 6,311 & 10,935 & 2,156 & 2,629 & 22,031 \\
\hline 2005 & 43,141 & 7,708 & 10,801 & 1,696 & 722 & 20,927 & 6,529 & 10,882 & 2,215 & 2,589 & 22,214 \\
\hline 2006 & 43,494 & 7,944 & 10,723 & 1,739 & 720 & 21,126 & 6,740 & 10,812 & 2,266 & 2,549 & 22,367 \\
\hline 2007 & 43,860 & 8,194 & 10,645 & 1,780 & 719 & 21,338 & 6,946 & 10,745 & 2,317 & 2,514 & 22,521 \\
\hline $16-19$ & & & & & & & & & & & \\
\hline 1971 & 2,666 & 1,327 & 34 & 0 & 0 & 1,362 & 1,163 & 142 & 0 & 0 & 1,305 \\
\hline 1976 & 2,901 & 1,454 & 28 & 0 & 0 & 1,482 & 1,289 & 129 & 0 & 0 & 1,419 \\
\hline 1981 & 3,310 & 1,675 & 20 & 0 & 0 & 1,694 & 1,523 & 93 & 0 & 0 & 1,616 \\
\hline 1986 & 3,131 & 1,587 & 10 & 0 & 0 & 1,596 & 1,484 & 49 & 1 & 0 & 1,535 \\
\hline 1991 & 2,665 & 1,358 & 8 & 0 & 0 & 1,366 & 1,267 & 32 & 0 & 0 & 1,300 \\
\hline 1996 & 2,402 & 1,209 & 6 & 0 & 0 & 1,216 & 1,164 & 21 & 0 & 0 & 1,186 \\
\hline 1999 & 2,543 & 1,280 & 6 & 1 & 1 & 1,288 & 1,234 & 20 & 1 & 1 & 1,255 \\
\hline 2000 & 2,523 & 1,276 & 6 & 1 & 1 & 1,283 & 1,221 & 18 & 1 & 1 & 1,240 \\
\hline 2001 & 2,567 & 1,304 & 5 & 1 & 1 & 1,312 & 1,237 & 16 & 1 & 1 & 1,255 \\
\hline 2002 & 2,630 & 1,352 & 4 & 1 & 1 & 1,357 & 1,259 & 13 & 1 & 1 & 1,273 \\
\hline 2003 & 2,703 & 1,392 & 4 & 1 & 1 & 1,397 & 1,293 & 12 & 0 & 1 & 1,306 \\
\hline 2004 & 2,771 & 1,424 & 3 & 0 & 0 & 1,428 & 1,332 & 11 & 0 & 0 & 1,343 \\
\hline 2005 & 2,801 & 1,434 & 2 & 0 & 0 & 1,436 & 1,355 & 9 & 0 & 0 & 1,365 \\
\hline 2006 & 2,829 & 1,457 & 2 & 0 & 0 & 1,459 & 1,364 & 7 & 0 & 0 & 1,370 \\
\hline 2007 & 2,861 & 1,474 & 2 & 0 & 0 & 1,475 & 1,380 & 6 & 0 & 0 & 1,386 \\
\hline $20-24$ & & & & & & & & & & & \\
\hline 1971 & 3,773 & 1,211 & 689 & 3 & 0 & 1,904 & 745 & 1,113 & 9 & 2 & 1,869 \\
\hline 1976 & 3,395 & 1,167 & 557 & 4 & 0 & 1,728 & 725 & 925 & 16 & 2 & 1,667 \\
\hline 1981 & 3,744 & 1,420 & 466 & 10 & 1 & 1,896 & 1,007 & 811 & 27 & 2 & 1,847 \\
\hline 1986 & 4,171 & 1,768 & 317 & 14 & 0 & 2,099 & 1,383 & 657 & 32 & 1 & 2,072 \\
\hline 1991 & 3,911 & 1,717 & 242 & 12 & 0 & 1,971 & 1,421 & 490 & 29 & 1 & 1,941 \\
\hline 1996 & 3,291 & 1,538 & 117 & 3 & 0 & 1,658 & 1,361 & 260 & 11 & 1 & 1,633 \\
\hline 1999 & 3,047 & 1,449 & 78 & 2 & 0 & 1,530 & 1,320 & 188 & 8 & 1 & 1,517 \\
\hline 2000 & 3,088 & 1,470 & 74 & 3 & 0 & 1,548 & 1,352 & 180 & 8 & 1 & 1,540 \\
\hline 2001 & 3,157 & 1,501 & 74 & 3 & 1 & 1,579 & 1,390 & 178 & 8 & 1 & 1,578 \\
\hline 2002 & 3,212 & 1,533 & 69 & 3 & 1 & 1,606 & 1,430 & 167 & 8 & 1 & 1,606 \\
\hline 2003 & 3,281 & 1,573 & 68 & 3 & 1 & 1,645 & 1,465 & 161 & 8 & 1 & 1,636 \\
\hline 2004 & 3,376 & 1,639 & 69 & 3 & 1 & 1,712 & 1,497 & 157 & 8 & 2 & 1,664 \\
\hline 2005 & 3,477 & 1,700 & 66 & 3 & 1 & 1,771 & 1,547 & 150 & 8 & 2 & 1,706 \\
\hline 2006 & 3,558 & 1,749 & 59 & 3 & 1 & 1,812 & 1,599 & 138 & 7 & 1 & 1,746 \\
\hline 2007 & 3,661 & 1,822 & 56 & 3 & 1 & 1,881 & 1,646 & 126 & 6 & 1 & 1,780 \\
\hline $25-29$ & & & & & & & & & & & \\
\hline 1971 & 3,267 & 431 & 1,206 & 16 & 1 & 1,654 & 215 & 1,367 & 29 & 4 & 1,614 \\
\hline 1976 & 3,758 & 533 & 1,326 & 39 & 2 & 1,900 & 267 & 1,522 & 65 & 5 & 1,859 \\
\hline 1981 & 3,372 & 588 & 1,057 & 54 & 1 & 1,700 & 331 & 1,247 & 89 & 4 & 1,671 \\
\hline 1986 & 3,713 & 835 & 949 & 79 & 1 & 1,863 & 527 & 1,207 & 113 & 4 & 1,850 \\
\hline 1991 & 4,154 & 1,132 & 856 & 82 & 1 & 2,071 & 800 & 1,158 & 123 & 2 & 2,083 \\
\hline 1996 & 3,950 & 1,273 & 650 & 46 & 1 & 1,970 & 977 & 906 & 93 & 3 & 1,980 \\
\hline 1999 & 3,687 & 1,304 & 497 & 34 & 1 & 1,836 & 1,051 & 725 & 72 & 3 & 1,851 \\
\hline 2000 & 3,605 & 1,305 & 459 & 31 & 1 & 1,796 & 1,065 & 677 & 65 & 3 & 1,810 \\
\hline 2001 & 3,487 & 1,293 & 420 & 28 & 1 & 1,742 & 1,059 & 625 & 58 & 3 & 1,745 \\
\hline 2002 & 3,365 & 1,286 & 375 & 26 & 1 & 1,688 & 1,054 & 568 & 52 & 3 & 1,676 \\
\hline 2003 & 3,284 & 1,281 & 340 & 25 & 1 & 1,647 & 1,060 & 527 & 49 & 2 & 1,638 \\
\hline 2004 & 3,280 & 1,297 & 319 & 24 & 1 & 1,641 & 1,089 & 501 & 47 & 2 & 1,639 \\
\hline 2005 & 3,354 & 1,344 & 307 & 23 & 1 & 1,675 & 1,143 & 488 & 46 & 2 & 1,679 \\
\hline 2006 & 3,434 & 1,400 & 295 & 23 & 1 & 1,718 & 1,198 & 471 & 46 & 2 & 1,716 \\
\hline 2007 & 3,526 & 1,457 & 289 & 23 & 1 & 1,770 & 1,248 & 461 & 45 & 2 & 1,756 \\
\hline
\end{tabular}




\begin{tabular}{|c|c|c|c|c|c|c|c|c|c|c|c|}
\hline \multirow{2}{*}{$\begin{array}{l}\text { Table } 1.5 \\
\text { continued } \\
\text { England and Wales }\end{array}$} & \multicolumn{11}{|c|}{ Population: age, sex and legal marital status } \\
\hline & \multirow{3}{*}{\begin{tabular}{|c|} 
Total \\
population
\end{tabular}} & & & & & & & & & Numbe & housands) \\
\hline & & \multicolumn{5}{|c|}{ Males } & \multicolumn{5}{|c|}{ Females } \\
\hline Mid-year & & Single & Married & Divorced & Widowed & Total & Single & Married & Divorced & Widowed & Total \\
\hline \multicolumn{12}{|l|}{$30-34$} \\
\hline 1971 & 2,897 & 206 & 1,244 & 23 & 3 & 1,475 & 111 & 1,269 & 34 & 8 & 1,422 \\
\hline 1976 & 3,220 & 236 & 1,338 & 55 & 3 & 1,632 & 118 & 1,388 & 75 & 8 & 1,588 \\
\hline 1981 & 3,715 & 318 & 1,451 & 97 & 3 & 1,869 & 165 & 1,544 & 129 & 9 & 1,846 \\
\hline 1986 & 3,338 & 355 & 1,197 & 124 & 2 & 1,679 & 206 & 1,293 & 154 & 6 & 1,660 \\
\hline 1991 & 3,708 & 520 & 1,172 & 155 & 2 & 1,849 & 335 & 1,330 & 189 & 5 & 1,859 \\
\hline 1996 & 4,126 & 776 & 1,135 & 138 & 2 & 2,050 & 551 & 1,316 & 201 & 7 & 2,076 \\
\hline 1999 & 4,113 & 877 & 1,043 & 121 & 3 & 2,044 & 651 & 1,223 & 188 & 7 & 2,069 \\
\hline 2000 & 4,076 & 904 & 1,007 & 114 & 2 & 2,027 & 679 & 1,182 & 181 & 7 & 2,049 \\
\hline 2001 & 4,050 & 934 & 971 & 108 & 2 & 2,016 & 711 & 1,142 & 174 & 7 & 2,033 \\
\hline 2002 & 3,992 & 959 & 918 & 105 & 2 & 1,984 & 742 & 1,093 & 167 & 6 & 2,009 \\
\hline 2003 & 3,919 & 979 & 864 & 102 & 2 & 1,947 & 766 & 1,041 & 159 & 6 & 1,972 \\
\hline 2004 & 3,810 & 988 & 810 & 97 & 2 & 1,897 & 777 & 982 & 149 & 5 & 1,913 \\
\hline 2005 & 3,724 & 1,002 & 761 & 92 & 2 & 1,856 & 791 & 933 & 139 & 5 & 1,868 \\
\hline 2006 & 3,606 & 1,010 & 703 & 84 & 2 & 1,799 & 800 & 876 & 127 & 5 & 1,808 \\
\hline 2007 & 3,473 & 1,010 & 649 & 78 & 2 & 1,738 & 800 & 814 & 117 & 4 & 1,735 \\
\hline \multicolumn{12}{|l|}{$35-44$} \\
\hline 1971 & 5,736 & 317 & 2,513 & 48 & 13 & 2,891 & 201 & 2,529 & 66 & 48 & 2,845 \\
\hline 1976 & 5,608 & 286 & 2,442 & 104 & 12 & 2,843 & 167 & 2,427 & 129 & 42 & 2,765 \\
\hline 1981 & 5,996 & 316 & 2,519 & 178 & 12 & 3,024 & 170 & 2,540 & 222 & 41 & 2,972 \\
\hline 1986 & 6,856 & 396 & 2,738 & 293 & 12 & 3,438 & 213 & 2,815 & 350 & 39 & 3,418 \\
\hline 1991 & 7,022 & 477 & 2,632 & 384 & 11 & 3,504 & 280 & 2,760 & 444 & 34 & 3,517 \\
\hline 1996 & 7,017 & 653 & 2,426 & 398 & 12 & 3,489 & 427 & 2,568 & 497 & 36 & 3,528 \\
\hline 1999 & 7,475 & 832 & 2,459 & 408 & 13 & 3,711 & 577 & 2,617 & 533 & 37 & 3,763 \\
\hline 2000 & 7,661 & 899 & 2,481 & 410 & 12 & 3,802 & 635 & 2,640 & 547 & 37 & 3,859 \\
\hline 2001 & 7,816 & 963 & 2,494 & 411 & 12 & 3,881 & 692 & 2,649 & 558 & 36 & 3,935 \\
\hline 2002 & 7,964 & 1,031 & 2,490 & 424 & 12 & 3,957 & 751 & 2,650 & 572 & 35 & 4,007 \\
\hline 2003 & 8,058 & 1,089 & 2,471 & 435 & 12 & 4,007 & 804 & 2,631 & 583 & 34 & 4,051 \\
\hline 2004 & 8,133 & 1,141 & 2,441 & 443 & 11 & 4,036 & 858 & 2,613 & 593 & 32 & 4,097 \\
\hline 2005 & 8,194 & 1,195 & 2,417 & 450 & 11 & 4,073 & 910 & 2,583 & 597 & 31 & 4,121 \\
\hline 2006 & 8,213 & 1,249 & 2,371 & 448 & 11 & 4,080 & 965 & 2,543 & 595 & 30 & 4,134 \\
\hline 2007 & 8,209 & 1,303 & 2,319 & 444 & 11 & 4,076 & 1,019 & 2,498 & 586 & 29 & 4,132 \\
\hline \multicolumn{12}{|l|}{$45-64$} \\
\hline 1971 & 11,887 & 502 & 4,995 & 81 & 173 & 5,751 & 569 & 4,709 & 125 & 733 & 6,136 \\
\hline 1976 & 11,484 & 496 & 4,787 & 141 & 160 & 5,583 & 462 & 4,568 & 188 & 683 & 5,901 \\
\hline 1981 & 11,040 & 480 & 4,560 & 218 & 147 & 5,405 & 386 & 4,358 & 271 & 620 & 5,635 \\
\hline 1986 & 10,860 & 461 & 4,422 & 331 & 141 & 5,355 & 327 & 4,220 & 388 & 570 & 5,505 \\
\hline 1991 & 10,960 & 456 & 4,394 & 456 & 127 & 5,433 & 292 & 4,211 & 521 & 503 & 5,527 \\
\hline 1996 & 11,820 & 528 & 4,587 & 628 & 121 & 5,864 & 318 & 4,466 & 732 & 440 & 5,956 \\
\hline 1999 & 12,198 & 589 & 4,627 & 706 & 121 & 6,043 & 355 & 4,541 & 844 & 415 & 6,155 \\
\hline 2000 & 12,328 & 615 & 4,638 & 727 & 121 & 6,101 & 372 & 4,564 & 881 & 410 & 6,227 \\
\hline 2001 & 12,447 & 644 & 4,647 & 747 & 121 & 6,159 & 391 & 4,578 & 918 & 401 & 6,289 \\
\hline 2002 & 12,573 & 670 & 4,642 & 779 & 120 & 6,211 & 413 & 4,597 & 960 & 391 & 6,362 \\
\hline 2003 & 12,710 & 702 & 4,643 & 814 & 119 & 6,278 & 437 & 4,612 & 1,002 & 381 & 6,432 \\
\hline 2004 & 12,852 & 736 & 4,643 & 850 & 117 & 6,347 & 465 & 4,625 & 1,045 & 371 & 6,505 \\
\hline 2005 & 13,021 & 774 & 4,652 & 888 & 117 & 6,431 & 497 & 4,642 & 1,090 & 362 & 6,590 \\
\hline 2006 & 13,243 & 818 & 4,676 & 926 & 117 & 6,537 & 535 & 4,677 & 1,138 & 356 & 6,706 \\
\hline 2007 & 13,439 & 864 & 4,678 & 963 & 116 & 6,621 & 579 & 4,704 & 1,187 & 348 & 6,818 \\
\hline \multicolumn{12}{|l|}{65 and over } \\
\hline 1971 & 6,592 & 179 & 1,840 & 17 & 492 & 2,527 & 580 & 1,437 & 32 & 2,016 & 4,065 \\
\hline 1976 & 7,119 & 197 & 2,033 & 33 & 510 & 2,773 & 569 & 1,579 & 60 & 2,138 & 4,347 \\
\hline 1981 & 7,548 & 216 & 2,167 & 54 & 534 & 2,971 & 533 & 1,692 & 90 & 2,263 & 4,578 \\
\hline 1986 & 7,768 & 223 & 2,234 & 76 & 539 & 3,072 & 477 & 1,759 & 127 & 2,333 & 4,696 \\
\hline 1991 & 8,080 & 231 & 2,332 & 99 & 586 & 3,248 & 422 & 1,853 & 152 & 2,405 & 4,832 \\
\hline 1996 & 8,221 & 247 & 2,390 & 134 & 597 & 3,367 & 369 & 1,897 & 196 & 2,393 & 4,854 \\
\hline 1999 & 8,262 & 251 & 2,431 & 161 & 594 & 3,437 & 338 & 1,922 & 230 & 2,336 & 4,825 \\
\hline 2000 & 8,287 & 252 & 2,449 & 171 & 593 & 3,466 & 327 & 1,938 & 243 & 2,313 & 4,821 \\
\hline 2001 & 8,342 & 254 & 2,478 & 183 & 595 & 3,510 & 318 & 1,960 & 259 & 2,295 & 4,832 \\
\hline 2002 & 8,398 & 255 & 2,508 & 196 & 594 & 3,554 & 309 & 1,987 & 276 & 2,272 & 4,844 \\
\hline 2003 & 8,454 & 257 & 2,538 & 210 & 593 & 3,597 & 301 & 2,017 & 295 & 2,245 & 4,857 \\
\hline 2004 & 8,510 & 258 & 2,566 & 224 & 592 & 3,640 & 293 & 2,046 & 314 & 2,216 & 4,870 \\
\hline 2005 & 8,571 & 260 & 2,596 & 239 & 590 & 3,685 & 286 & 2,077 & 335 & 2,187 & 4,885 \\
\hline 2006 & 8,611 & 261 & 2,618 & 254 & 589 & 3,722 & 279 & 2,101 & 353 & 2,155 & 4,889 \\
\hline 2007 & 8,690 & 264 & 2,652 & 270 & 589 & 3,775 & 274 & 2,137 & 375 & 2,128 & 4,915 \\
\hline
\end{tabular}




\begin{tabular}{|c|c|c|c|c|c|c|c|c|c|c|c|c|c|c|c|c|c|c|}
\hline Table 2.1 & Vital s & 5 & summar & & & & & & & & & & & & & & & \\
\hline Enstituent & sof the Uni & ted Kingo & & & & & & & & & & & & & Numb & oers (thou & usands) an & nd rates \\
\hline $\begin{array}{l}\text { Year and } \\
\text { quarter }\end{array}$ & $\begin{array}{l}\text { All I } \\
\text { birt }\end{array}$ & & $\begin{array}{r}\text { Live b } \\
\text { outside } m\end{array}$ & \begin{tabular}{|l|} 
irths \\
narriage
\end{tabular} & Marric & & $\begin{array}{r}\text { Civ } \\
\text { Partner }\end{array}$ & il & Divor & & Dea & & $\begin{array}{r}\text { Infa } \\
\text { mortc }\end{array}$ & & $\begin{array}{l}\text { Neon } \\
\text { morta }\end{array}$ & & & $\begin{array}{l}\text { natal } \\
\text { tality }\end{array}$ \\
\hline & Number & $\begin{array}{l}\text { Rate' } \\
\end{array}$ & Number & \begin{tabular}{l|l} 
Rate $^{2}$ \\
\end{tabular} & \begin{tabular}{|l|} 
Number \\
\end{tabular} & $\begin{array}{l}\text { Rate }^{3} \\
\end{array}$ & Number & $\begin{array}{l}\text { Rate }^{4} \\
\end{array}$ & Number & $\begin{array}{l}\text { Rate }^{5} \\
\end{array}$ & \begin{tabular}{|l|} 
Number \\
\end{tabular} & $\begin{array}{l}\text { Rate }^{1} \\
\end{array}$ & Number & Rate $^{2}$ & Number & \begin{tabular}{l|} 
Rate $^{2}$ \\
\end{tabular} & \begin{tabular}{|l|} 
Number \\
\end{tabular} & \begin{tabular}{|l|l|} 
Rate $^{9}$ \\
\end{tabular} \\
\hline $\begin{array}{l}\text { United Kingdom } \\
1976 \\
1981 \\
1986 \\
1991 \\
1996\end{array}$ & $\begin{array}{l}675.5 \\
730.7 \\
754.8 \\
792.3 \\
733.2\end{array}$ & $\begin{array}{l}12.0 \\
13.0 \\
13.3 \\
13.8 \\
12.6\end{array}$ & $\begin{array}{r}61.1 \\
91.3 \\
154.3 \\
236.1 \\
260.4\end{array}$ & $\begin{array}{r}90 \\
125 \\
204 \\
298 \\
355\end{array}$ & $\begin{array}{l}406.0 \\
399.8 \\
399.9 \\
349.7 \\
317.5\end{array}$ & $\begin{array}{c}49.4 \\
: . \\
. .\end{array}$ & & & $\begin{array}{l}135.4 \\
156.4 \\
168.2 \\
173.5 \\
171.7\end{array}$ & $\begin{array}{c}11.3 \\
. . \\
. .\end{array}$ & $\begin{array}{l}680.8 \\
658.0 \\
660.7 \\
646.2 \\
636.0\end{array}$ & $\begin{array}{l}12.1 \\
11.7 \\
11.7 \\
11.2 \\
10.9\end{array}$ & $\begin{array}{l}9.79 \\
8.16 \\
7.18 \\
5.52 \\
4.50\end{array}$ & $\begin{array}{l}14.5 \\
11.2 \\
9.5 \\
7.4 \\
6.1\end{array}$ & $\begin{array}{l}6.68 \\
4.93 \\
4.00 \\
3.46 \\
3.00\end{array}$ & $\begin{array}{l}9.9 \\
6.7 \\
5.3 \\
4.4 \\
4.1\end{array}$ & $\begin{array}{r}12.25 \\
87.79 \\
6.45 \\
6.41\end{array}$ & $\begin{array}{r}18.0 \\
12.0 \\
9.6 \\
8.1 \\
8.7\end{array}$ \\
\hline $\begin{array}{l}1999 \\
2000 \\
2001 \\
2002 \\
2003\end{array}$ & $\begin{array}{l}700.0 \\
679.0 \\
669.1 \\
668.8 \\
695.6\end{array}$ & $\begin{array}{l}11.9 \\
11.5 \\
11.3 \\
11.3 \\
11.7\end{array}$ & $\begin{array}{l}271.6 \\
268.1 \\
268.0 \\
271.7 \\
288.5\end{array}$ & $\begin{array}{l}388 \\
395 \\
401 \\
406 \\
415\end{array}$ & $\begin{array}{l}301.1 \\
30.9 \\
286.1 \\
299.0 \\
308.6\end{array}$ & 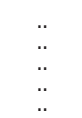 & . & & $\begin{array}{l}158.7 \\
154.6 \\
156.8 \\
160.5 \\
166.7\end{array}$ & $\begin{array}{l}. . \\
. . \\
. .\end{array}$ & $\begin{array}{l}632.1 \\
608.4 \\
602.3 \\
606.2 \\
612.0\end{array}$ & $\begin{array}{l}10.8 \\
10.3 \\
10.2 \\
10.2 \\
10.3\end{array}$ & $\begin{array}{l}4.05 \\
3.81 \\
33.66 \\
3.54 \\
3.69\end{array}$ & $\begin{array}{l}5.8 \\
5.6 \\
5.5 \\
5.3 \\
5.3\end{array}$ & $\begin{array}{l}2.73 \\
2.63 \\
2.44 \\
2.37 \\
2.54\end{array}$ & $\begin{array}{l}3.9 \\
3.9 \\
3.9 \\
3.6 \\
3.7\end{array}$ & $\begin{array}{l}5.79 \\
5.56 \\
5.39 \\
5.53 \\
5.92\end{array}$ & $\begin{array}{l}8.2 \\
8.1 \\
8.0 \\
8.2 \\
8.5\end{array}$ \\
\hline $\begin{array}{l}2004 \\
2005 \\
2006 \\
2007\end{array}$ & $\begin{array}{l}716.0 \\
722.5 \\
748.6 \\
772.2\end{array}$ & $\begin{array}{l}12.0 \\
12.0 \\
12.4 \\
12.7\end{array}$ & $\begin{array}{l}302.6 \\
310.2 \\
326.8 \\
343.2\end{array}$ & $\begin{array}{l}423 \\
429 \\
437 \\
444\end{array}$ & $\begin{array}{l}313.6 \\
286.6 \\
277.6^{p} \\
270.0^{p}\end{array}$ & $\begin{array}{l}. \\
\because\end{array}$ & $\begin{array}{c}1.955^{10} \\
16.11^{\mathrm{p}} \\
8.73^{\mathrm{P}}\end{array}$ & 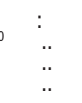 & $\begin{array}{l}167.1 \\
155.1 \\
148.1 \\
1442^{p}\end{array}$ & $\begin{array}{l}. . \\
.\end{array}$ & $\begin{array}{l}583.1 \\
582.7 \\
572.2 \\
574.7\end{array}$ & $\begin{array}{l}9.7 \\
9.7 \\
9.4\end{array}$ & $\begin{array}{l}3.66 \\
3.68 \\
3.74 \\
3.74\end{array}$ & $\begin{array}{l}5.1 \\
5.1 \\
5.0 \\
4.8\end{array}$ & $\begin{array}{l}2.49 \\
2.52 \\
2.61 \\
2.55\end{array}$ & $\begin{array}{l}3.5 \\
3.5 \\
3.5 \\
3.3\end{array}$ & $\begin{array}{l}5.88 \\
5.78 \\
5.94 \\
6.02\end{array}$ & $\begin{array}{l}8.2 \\
8.0 \\
7.9 \\
7.8\end{array}$ \\
\hline 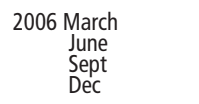 & $\begin{array}{l}178.9 \\
186.0 \\
195.2 \\
188.5\end{array}$ & $\begin{array}{l}12.0 \\
12.3 \\
12.8 \\
12.3\end{array}$ & $\begin{array}{l}77.5 \\
80.2 \\
85.8 \\
83.3\end{array}$ & $\begin{array}{l}433 \\
431 \\
439 \\
442\end{array}$ & $\begin{array}{r}30.3^{p} \\
17.6^{p} \\
12.9^{p} \\
48.6^{p}\end{array}$ & $\begin{array}{l}\because \\
\because\end{array}$ & $\begin{array}{l}4.87 \\
4.36 \\
4.49 \\
2.38\end{array}$ & . & $\begin{array}{l}37.7 \\
36.7 \\
37.0 \\
36.7\end{array}$ & $\begin{array}{l}. . \\
\ddot{.} \\
.\end{array}$ & $\begin{array}{l}159.9 \\
141.4 \\
130.7 \\
140.2\end{array}$ & $\begin{array}{l}10.7 \\
9.4 \\
8.6 \\
9.2\end{array}$ & $\begin{array}{l}0.99 \\
0.94 \\
0.93 \\
0.97\end{array}$ & $\begin{array}{l}5.1 \\
5.0 \\
4.8 \\
5.2\end{array}$ & $\begin{array}{l}0.61 \\
0.65 \\
0.67 \\
0.68\end{array}$ & $\begin{array}{l}3.4 \\
3.5 \\
3.4 \\
3.6\end{array}$ & $\begin{array}{l}1.45 \\
1.50 \\
1.54 \\
1.45\end{array}$ & $\begin{array}{l}8.1 \\
8.0 \\
7.8 \\
7.7\end{array}$ \\
\hline $\begin{array}{c}2007 \text { March } \\
\text { June } \\
\text { Sept } \\
\text { Dec }\end{array}$ & $\begin{array}{l}184.4 \\
189.8 \\
202.8 \\
195.3\end{array}$ & $\begin{array}{l}12.3 \\
12.5 \\
13.2 \\
12.7\end{array}$ & $\begin{array}{l}81.9 \\
82.6 \\
90.5 \\
88.1\end{array}$ & $\begin{array}{l}444 \\
435 \\
446 \\
451\end{array}$ & $\begin{array}{r}31.0^{p} \\
15.7^{p} \\
15.8^{p} \\
47.4^{p}\end{array}$ & :. & $\begin{array}{l}1.69 \\
2.37 \\
2.96 \\
1.71\end{array}$ & " & $\begin{array}{l}38.9^{p} \\
37.2^{p} \\
33.7^{p} \\
31.4^{p}\end{array}$ & $\begin{array}{l}. . \\
\ddot{.}\end{array}$ & $\begin{array}{l}159.3 \\
138.0 \\
129.9 \\
147.5\end{array}$ & $\begin{array}{l}10.6 \\
9.1 \\
8.4 \\
9.6\end{array}$ & $\begin{array}{l}0.91 \\
0.99 \\
0.96 \\
0.90\end{array}$ & $\begin{array}{l}4.9 \\
5.2 \\
4.6 \\
4.6\end{array}$ & $\begin{array}{l}0.64 \\
0.66 \\
0.63 \\
0.62\end{array}$ & $\begin{array}{l}3.4 \\
3.5 \\
3.1 \\
3.2\end{array}$ & $\begin{array}{l}1.47 \\
1.52 \\
1.51 \\
1.52\end{array}$ & $\begin{array}{l}7.9 \\
8.0 \\
7.4 \\
7.7\end{array}$ \\
\hline $\begin{array}{c}2008 \text { March } \\
\text { June } \\
\text { Sept }\end{array}$ & $\begin{array}{l}195.4^{p} \\
198.0^{p} \\
204.0^{\circ}\end{array}$ & $\begin{array}{l}12 . .^{p} \\
12.5^{p} \\
13.3^{p}\end{array}$ & $\begin{array}{l}88.8^{p} \\
82.6^{p} \\
93.0^{p}\end{array}$ & $\begin{array}{l}455^{p} \\
449^{p} \\
456^{p}\end{array}$ & .. & $\begin{array}{l}. . \\
. .\end{array}$ & $\begin{array}{l}1.25^{p} \\
1.94^{p}\end{array}$ &. & $\begin{array}{l}. . \\
. .\end{array}$ & $\begin{array}{l}. . \\
. .\end{array}$ & $\begin{array}{l}155.4^{p} \\
139.8^{p} \\
129.8^{p}\end{array}$ & $\begin{array}{c}10.2^{p} \\
9.2^{p} \\
8.4^{p}\end{array}$ & $\begin{array}{l}0.95^{p} \\
0.82^{p} \\
0.96^{p}\end{array}$ & $\begin{array}{l}4.9^{p} \\
4.2^{p} \\
4.7^{p}\end{array}$ & $\begin{array}{l}0.66^{p} \\
0.59^{p} \\
0.68^{p}\end{array}$ & $\begin{array}{l}3.4^{p} \\
3.0^{p} \\
3.3^{p}\end{array}$ & $\begin{array}{l}1.52^{p} \\
1.47^{p} \\
1.46^{p}\end{array}$ & $\begin{array}{l}7.7^{p} \\
7.4^{p} \\
7.1^{p}\end{array}$ \\
\hline $\begin{array}{l}\text { England and Wales } \\
1916 \\
1981 \\
1986 \\
1991 \\
1996\end{array}$ & $\begin{array}{l}584.3 \\
634.5 \\
661.0 \\
699.2 \\
649.5\end{array}$ & $\begin{array}{l}11.8 \\
12.8 \\
13.2 \\
13.8 \\
12.6\end{array}$ & $\begin{array}{r}53.8 \\
81.0 \\
141.3 \\
211.3 \\
232.7\end{array}$ & $\begin{array}{r}92 \\
128 \\
214 \\
302 \\
358\end{array}$ & $\begin{array}{l}358.6 \\
352.0 \\
347.9 \\
30.8 \\
279.0\end{array}$ & $\begin{array}{l}57.7 \\
49.6 \\
43.6 \\
36.0 \\
30.9\end{array}$ & & & $\begin{array}{l}126.7 \\
145.7 \\
153.9 \\
158.7 \\
157.1\end{array}$ & $\begin{array}{l}10.1 \\
11.9 \\
12.9 \\
13.5 \\
13.8\end{array}$ & $\begin{array}{l}598.5 \\
577.9 \\
581.2 \\
570.0 \\
560.1\end{array}$ & $\begin{array}{l}12.1 \\
11.6 \\
11.6 \\
11.2 \\
10.9\end{array}$ & $\begin{array}{l}8.34 \\
7.02 \\
6.31 \\
5.16 \\
3.99\end{array}$ & $\begin{array}{l}14.3 \\
11.1 \\
9.6 \\
7.4 \\
6.1\end{array}$ & $\begin{array}{l}5.66 \\
4.23 \\
3.49 \\
3.05 \\
2.68\end{array}$ & $\begin{array}{l}9.7 \\
6.7 \\
5.3 \\
4.4 \\
4.1\end{array}$ & $\begin{array}{l}10.45 \\
7.56 \\
6.37 \\
5.65 \\
5.62\end{array}$ & $\begin{array}{l}17.7 \\
11.8 \\
9.6 \\
8.0 \\
8.6\end{array}$ \\
\hline $\begin{array}{l}1999 \\
2000 \\
2001 \\
22002 \\
2003\end{array}$ & $\begin{array}{l}621.9 \\
6044.4 \\
594.6 \\
596.1 \\
621.5\end{array}$ & $\begin{array}{l}12.0 \\
11.6 \\
11.4 \\
11.3 \\
11.8\end{array}$ & $\begin{array}{l}241.9 \\
238.6 \\
238.1 \\
242.0 \\
257.2\end{array}$ & $\begin{array}{l}389 \\
395 \\
400 \\
406 \\
414\end{array}$ & $\begin{array}{l}263.5 \\
26.5 \\
249.2 \\
255.6 \\
270.1\end{array}$ & $\begin{array}{l}27.8 \\
27.8 \\
25.4 \\
25.6 \\
26.4\end{array}$ & & & $\begin{array}{l}144.6 \\
141.1 \\
143.8 \\
147.7 \\
153.5\end{array}$ & $\begin{array}{l}12.9 \\
12.7 \\
12.9 \\
13.4 \\
14.0\end{array}$ & $\begin{array}{l}556.1 \\
555.7 \\
530.4 \\
533.5 \\
538.3\end{array}$ & $\begin{array}{l}10.7 \\
10.3 \\
10.1 \\
10.1 \\
10.2\end{array}$ & $\begin{array}{l}3.62 \\
3.38 \\
3.24 \\
3.13 \\
3.31\end{array}$ & $\begin{array}{l}5.8 \\
5.6 \\
5.4 \\
5.2 \\
5.3\end{array}$ & $\begin{array}{l}2.44 \\
2.34 \\
2.14 \\
2.13 \\
2.26\end{array}$ & $\begin{array}{l}3.9 \\
3.9 \\
3.6 \\
3.6 \\
3.6\end{array}$ & $\begin{array}{l}5.14 \\
4.96 \\
4.76 \\
4.99 \\
5.36\end{array}$ & $\begin{array}{l}8.2 \\
8.2 \\
8.0 \\
8.3 \\
8.6\end{array}$ \\
\hline $\begin{array}{l}2004 \\
2005 \\
2006 \\
2007\end{array}$ & $\begin{array}{l}639.7 \\
6455.8 \\
669.6 \\
690.0\end{array}$ & $\begin{array}{l}12.1 \\
12.1 \\
12.1 \\
12.8\end{array}$ & $\begin{array}{l}269.7 \\
2276.5 \\
291.4 \\
305.6\end{array}$ & $\begin{array}{l}422 \\
428 \\
435 \\
443\end{array}$ & $\begin{array}{l}273.1 \\
2747.8 \\
239.0^{p} \\
231.4^{p}\end{array}$ & $\begin{array}{l}26.1 \\
23.1 \\
21.1 .1 \\
20.6^{p}\end{array}$ & $\begin{array}{c}1.866^{10} \\
14.94 \\
7.93\end{array}$ & 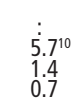 & $\begin{array}{l}153.4 \\
141.8 \\
132.6 \\
128.5^{\mathrm{p}}\end{array}$ & $\begin{array}{l}14.1 \\
13.1 \\
12.2 \\
12.0^{\circ}\end{array}$ & $\begin{array}{l}512.5 \\
552.7 \\
502.6 \\
504.1\end{array}$ & $\begin{array}{l}9.7 \\
9.7 \\
9.4 \\
9.3\end{array}$ & $\begin{array}{l}3.22 \\
3.26 \\
3.37 \\
3.35\end{array}$ & $\begin{array}{l}5.0 \\
5.0 \\
5.0 \\
4.8\end{array}$ & $\begin{array}{l}2.21 \\
2.23 \\
2.35 \\
2.28\end{array}$ & $\begin{array}{l}3.5 \\
3.4 \\
3.5 \\
3.3\end{array}$ & $\begin{array}{l}5.39 \\
5.21 \\
5.36 \\
5.39\end{array}$ & $\begin{array}{l}8.4 \\
8.0 \\
8.0 \\
7.8\end{array}$ \\
\hline $\begin{aligned} & 2006 \text { March } \\
& \text { June } \\
& \text { Sept } \\
& \text { Dec }\end{aligned}$ & $\begin{array}{l}159.5 \\
16.6 .2 \\
179.9 \\
169.0\end{array}$ & $\begin{array}{l}12.0 \\
12.4 \\
12.9 \\
12.5\end{array}$ & $\begin{array}{l}68.7 \\
71.4 \\
76.8 \\
74.5\end{array}$ & $\begin{array}{l}431 \\
430 \\
439 \\
441\end{array}$ & $\begin{array}{c}25.9^{p} \\
66.3^{p} \\
10.2^{p} \\
41.2^{p}\end{array}$ & $\begin{array}{l}9.6^{p} \\
24.2^{p} \\
38.4 p^{p} \\
14.9^{p}\end{array}$ & $\begin{array}{l}4.58 \\
4.01 \\
4.18 \\
2.18\end{array}$ & $\begin{array}{l}1.7 \\
1.5 \\
1.5 \\
0.8\end{array}$ & $\begin{array}{l}34.3 \\
33.0 \\
32.9 \\
32.4\end{array}$ & $\begin{array}{l}12.8 \\
12.2 \\
12.0 \\
11.8\end{array}$ & $\begin{array}{l}141.0 \\
123.9 \\
114.6 \\
123.1\end{array}$ & $\begin{array}{r}10.6 \\
9.2 \\
8.5 \\
9.1\end{array}$ & $\begin{array}{l}0.82 \\
0.84 \\
0.85 \\
0.86\end{array}$ & $\begin{array}{l}5.2 \\
5.1 \\
4.8 \\
5.1\end{array}$ & $\begin{array}{l}0.56 \\
0.58 \\
0.60 \\
0.60\end{array}$ & $\begin{array}{l}3.5 \\
3.5 \\
3.4 \\
3.6\end{array}$ & $\begin{array}{l}1.32 \\
1.37 \\
1.38 \\
1.30\end{array}$ & $\begin{array}{l}8.2 \\
8.2 \\
7.9 \\
7.6\end{array}$ \\
\hline $\begin{array}{l}2007 \text { March } \\
\text { June } \\
\text { Sept } \\
\text { Dec }\end{array}$ & $\begin{array}{l}164.0 \\
169.5 \\
181.4 \\
175.0\end{array}$ & $\begin{array}{l}12.3 \\
12.6 \\
13.3 \\
12.8\end{array}$ & $\begin{array}{l}72.5 \\
73.5 \\
80.8 \\
78.7\end{array}$ & 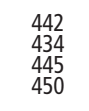 & $\begin{array}{l}26.77^{p} \\
65.2^{p} \\
99.4^{p} \\
40.1^{p}\end{array}$ & $\begin{array}{c}9.6^{p} \\
23.3^{p} \\
35.1^{p} \\
14.2^{p}\end{array}$ & $\begin{array}{l}1.55 \\
2.16 \\
2.68 \\
1.54\end{array}$ & $\begin{array}{l}0.6 \\
0.8 \\
0.9 \\
0.5\end{array}$ & $\begin{array}{l}34.77^{p} \\
33 . p^{p} \\
33.0^{p} \\
27.6^{p}\end{array}$ & $\begin{array}{l}13.2^{\mathrm{p}} \\
12.4^{\mathrm{p}} \\
12 . \mathrm{3}^{\mathrm{p}} \\
10.3^{\mathrm{p}}\end{array}$ & $\begin{array}{l}139.3 \\
121.0 \\
114.0 \\
129.7\end{array}$ & $\begin{array}{l}10.5 \\
9.0 \\
8.4 \\
9.5\end{array}$ & $\begin{array}{l}0.80 \\
0.88 \\
0.84 \\
0.83\end{array}$ & $\begin{array}{l}4.9 \\
5.2 \\
4.6 \\
4.7\end{array}$ & $\begin{array}{l}0.56 \\
0.60 \\
0.57 \\
0.56\end{array}$ & $\begin{array}{l}3.4 \\
3.5 \\
3.1 \\
3.2\end{array}$ & $\begin{array}{l}1.31 \\
1.36 \\
1.36 \\
1.37\end{array}$ & $\begin{array}{l}7.9 \\
8.0 \\
7.4 \\
7.8\end{array}$ \\
\hline $\begin{array}{c}2008 \text { March } \\
\text { June } \\
\text { Sept }\end{array}$ & $\begin{array}{l}173.8^{p} \\
176.8^{p} \\
182.0^{p}\end{array}$ & $\begin{array}{l}12 . .^{p} \\
13 . .^{p} \\
13.3^{p}\end{array}$ & $\begin{array}{l}78.5^{p} \\
79.3^{p} \\
82.8^{p}\end{array}$ & $\begin{array}{l}452^{\circ} \\
449^{\circ} \\
455^{\circ}\end{array}$ & .. & .. & $\begin{array}{l}1.13^{\mathrm{p}} \\
1.80^{\circ}\end{array}$ & $\begin{array}{l}0.4^{p} \\
0.6^{p}\end{array}$ & $\begin{array}{l}31.9^{p} \\
30.8^{p}\end{array}$ & $\begin{array}{l}12.0^{p} \\
11.6^{p}\end{array}$ & $\begin{array}{l}136.1^{p} \\
122.7^{p} \\
113.8^{p}\end{array}$ & $\begin{array}{c}10.1^{p} \\
9.1^{p} \\
8.3^{p}\end{array}$ & $\begin{array}{l}0.86^{p} \\
0.79^{p} \\
0.86^{p}\end{array}$ & $\begin{array}{l}5.0^{p} \\
4.5^{p} \\
4.7^{p}\end{array}$ & $\begin{array}{l}0.60^{\mathrm{p}} \\
0.53^{\mathrm{p}} \\
0.60^{\mathrm{p}}\end{array}$ & $\begin{array}{l}3.4^{p} \\
3.0^{p} \\
3.3^{p}\end{array}$ & $\begin{array}{l}1.36^{\mathrm{p}} \\
1.32^{\mathrm{p}} \\
1.30^{\mathrm{p}}\end{array}$ & $\begin{array}{l}7.9^{p} \\
7.5^{p} \\
7.1^{p}\end{array}$ \\
\hline $\begin{array}{l}\text { England } \\
1976 \\
1981 \\
1986 \\
1991 \\
1996\end{array}$ & $\begin{array}{l}550.4 \\
598.2 \\
662.6 \\
660.8 \\
614.2\end{array}$ & $\begin{array}{l}11.8 \\
12.8 \\
13.2 \\
13.8 \\
12.7\end{array}$ & $\begin{array}{r}50.8 \\
73.9 \\
13.5 \\
198.9 \\
218.2\end{array}$ & $\begin{array}{r}92 \\
129 \\
214 \\
301 \\
355\end{array}$ & $\begin{array}{l}339.0 \\
332.2 \\
328.4 \\
290.1 \\
264.2\end{array}$ & $\begin{array}{l}. . \\
\ddot{.} \\
. .\end{array}$ & & & $\begin{array}{l}146.0 \\
150.1 \\
148.7\end{array}$ & $\begin{array}{l}. . \\
. \\
.\end{array}$ & $\begin{array}{l}560.3 \\
541.0 \\
544.5 \\
534.0 \\
524.0\end{array}$ & $\begin{array}{l}12.0 \\
11.6 \\
11.6 \\
11.2 \\
10.8\end{array}$ & $\begin{array}{l}7.83 \\
6.50 \\
5.92 \\
4.86 \\
3.74\end{array}$ & $\begin{array}{r}14.2 \\
10.9 \\
9.5 \\
7.3 \\
6.1\end{array}$ & $\begin{array}{l}5.32 \\
3.93 \\
3.27 \\
2.87 \\
2.53\end{array}$ & $\begin{array}{l}9.7 \\
6.6 \\
5.2 \\
4.3 \\
4.1\end{array}$ & $\begin{array}{l}9.81 \\
7.04 \\
5.98 \\
5.33 \\
5.36\end{array}$ & $\begin{array}{r}17.6 \\
11.7 \\
9.5 \\
8.0 \\
8.7\end{array}$ \\
\hline $\begin{array}{l}1999 \\
2000 \\
2001 \\
2002 \\
2003\end{array}$ & $\begin{array}{l}589.5 \\
572.8 \\
563.7 \\
565.7 \\
589.9\end{array}$ & $\begin{array}{l}12.0 \\
11.7 \\
11.4 \\
11.4 \\
11.8\end{array}$ & $\begin{array}{l}226.7 \\
2223.8 \\
223.3 \\
2227.0 \\
241.4\end{array}$ & $\begin{array}{l}385 \\
391 \\
396 \\
401 \\
409\end{array}$ & $\begin{array}{l}249.5 \\
255.8 \\
236.2 \\
242.1 \\
255.6\end{array}$ & $\begin{array}{l}. \\
. \\
. .\end{array}$ & & & $\begin{array}{l}137.0 \\
133.9 \\
136.4 \\
140.2 \\
145.8\end{array}$ &.. & $\begin{array}{l}519.6 \\
501.0 \\
496.1 \\
499.1 \\
503.4\end{array}$ & $\begin{array}{l}10.8 \\
10.2 \\
10.0 \\
10.1 \\
10.1\end{array}$ & $\begin{array}{l}3.38 \\
3.18 \\
3.04 \\
2.97 \\
3.14\end{array}$ & $\begin{array}{l}5.7 \\
5.6 \\
5.4 \\
5.2 \\
5.3\end{array}$ & $\begin{array}{l}2.29 \\
2.21 \\
2.02 \\
2.022 \\
2.15\end{array}$ & $\begin{array}{l}3.9 \\
3.9 \\
3.6 \\
3.6 \\
3.7\end{array}$ & $\begin{array}{l}4.86 \\
4.69 \\
4.51 \\
4.75 \\
5.09\end{array}$ & $\begin{array}{l}8.2 \\
8.2 \\
8.0 \\
8.3 \\
8.6\end{array}$ \\
\hline $\begin{array}{l}2004 \\
2005 \\
2006 \\
2007\end{array}$ & $\begin{array}{l}607.2 \\
613.0 \\
635.7 \\
655.4\end{array}$ & $\begin{array}{l}12.1 \\
12.1 \\
12.5 \\
12.8\end{array}$ & $\begin{array}{l}253.1 \\
259.4 \\
27.5 \\
287.0\end{array}$ & $\begin{array}{l}417 \\
423 \\
430 \\
438^{\circ}\end{array}$ & $\begin{array}{l}258.2 \\
233.2 \\
222.0^{p} \\
215.6^{p}\end{array}$ & 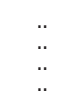 & $\begin{array}{c}i .7910 \\
14.38 \\
7.64\end{array}$ & : & $\begin{array}{l}145.5 \\
134.6 \\
125.6 \\
121.9^{p}\end{array}$ & .. & $\begin{array}{l}479.2 \\
479.4 \\
470.3 \\
470.7\end{array}$ & $\begin{array}{l}9.6 \\
9.6 \\
9.3 \\
9.2\end{array}$ & $\begin{array}{l}3.03 \\
3.10 \\
3.19 \\
3.13\end{array}$ & $\begin{array}{l}5.0 \\
5.0 \\
5.0 \\
4.8\end{array}$ & $\begin{array}{l}2.09 \\
2.12 \\
2.24 \\
2.15\end{array}$ & $\begin{array}{l}3.4 \\
3.5 \\
3.5 \\
3.3\end{array}$ & $\begin{array}{l}5.10 \\
4.92 \\
5.11 \\
5.10\end{array}$ & $\begin{array}{l}8.4 \\
8.0 \\
8.0 \\
7.7\end{array}$ \\
\hline $\begin{array}{c}2006 \text { March } \\
\text { June } \\
\text { Sept } \\
\text { Dec }\end{array}$ & $\begin{array}{l}151.4 \\
157.8 \\
166.0 \\
160.5\end{array}$ & $\begin{array}{l}12.1 \\
12.5 \\
13.0 \\
12.5\end{array}$ & $\begin{array}{l}64.5 \\
67.0 \\
72.0 \\
70.0\end{array}$ & $\begin{array}{l}426 \\
425 \\
434 \\
436\end{array}$ & $\begin{array}{c}24.4^{p} \\
2^{2} .5^{p} \\
13.19^{p} \\
38.9^{p}\end{array}$ & .. & $\begin{array}{l}4.42 \\
3.86 \\
4.02 \\
2.09\end{array}$ & .. & $\begin{array}{l}32.5 \\
31.2 \\
31.2 \\
30.7\end{array}$ &.. & $\begin{array}{l}132.0 \\
115.9 \\
107.1 \\
115.3\end{array}$ & $\begin{array}{l}10.5 \\
9.2 \\
8.4 \\
9.0\end{array}$ & $\begin{array}{l}0.79 \\
0.80 \\
0.80 \\
0.81\end{array}$ & $\begin{array}{l}5.2 \\
5.1 \\
4.8 \\
5.0\end{array}$ & $\begin{array}{l}0.54 \\
0.56 \\
0.57 \\
0.57\end{array}$ & $\begin{array}{l}3.6 \\
3.5 \\
3.4 \\
3.6\end{array}$ & $\begin{array}{l}1.26 \\
1.31 \\
1.31 \\
1.24\end{array}$ & $\begin{array}{l}8.3 \\
8.2 \\
7.8 \\
7.7\end{array}$ \\
\hline $\begin{array}{l}2007 \text { March } \\
\text { June } \\
\text { Sept } \\
\text { Dec }\end{array}$ & $\begin{array}{l}155.9 \\
161.0 \\
172.2 \\
166.3\end{array}$ & $\begin{array}{l}12.4 \\
12.6 \\
13.4 \\
12.9\end{array}$ & $\begin{array}{l}68.1 \\
69.0 \\
75.9 \\
74.0\end{array}$ & $\begin{array}{l}437 \\
429 \\
441 \\
445\end{array}$ & $\begin{array}{l}25.4 p^{p} \\
61.6 p^{p} \\
93.7^{p} \\
37.9^{p}\end{array}$ & $\begin{array}{l}. . \\
.\end{array}$ & $\begin{array}{l}1.49 \\
2.06 \\
2.60 \\
1.48\end{array}$ &.. & $\begin{array}{l}32.9^{p} \\
31.4{ }^{p} \\
31.4 \\
26.2^{p}\end{array}$ & .. & $\begin{array}{l}130.3 \\
112.9 \\
106.4 \\
121.2\end{array}$ & $\begin{array}{c}10.3 \\
8.9 \\
8.3 \\
9.4\end{array}$ & $\begin{array}{l}0.74 \\
0.84 \\
0.79 \\
0.76\end{array}$ & $\begin{array}{l}4.7 \\
5.2 \\
4.6 \\
4.6\end{array}$ & $\begin{array}{l}0.52 \\
0.57 \\
0.54 \\
0.53\end{array}$ & $\begin{array}{l}3.3 \\
3.5 \\
3.1 \\
3.2\end{array}$ & $\begin{array}{l}1.23 \\
1.30 \\
1.29 \\
1.28\end{array}$ & $\begin{array}{l}7.8 \\
8.0 \\
7.5 \\
7.6\end{array}$ \\
\hline $\begin{array}{c}2008 \text { March } \\
\text { June } \\
\text { Sept }\end{array}$ & $\begin{array}{l}165.0^{\circ} \\
167.8^{\circ} \\
172.8^{8}\end{array}$ & $\begin{array}{l}12.9^{p} \\
13.9^{p} \\
13.4^{p}\end{array}$ & $\begin{array}{l}73.7^{p} \\
74.3^{p} \\
77.7^{p}\end{array}$ & $\begin{array}{l}447^{p} \\
443^{p} \\
450^{p}\end{array}$ & $\ddot{.}$ & $\begin{array}{l}\ddot{.} \\
. .\end{array}$ & $\begin{array}{l}1.08^{\mathrm{p}} \\
1.73^{\mathrm{p}}\end{array}$ & $\begin{array}{l}. . \\
. .\end{array}$ & $\begin{array}{l}30.3^{p} \\
29.2^{p}\end{array}$ & .: & $\begin{array}{l}127.3^{p} \\
114.7^{p} \\
106.5^{p}\end{array}$ & $\begin{array}{l}9.9^{p} \\
9.0^{p} \\
8.2^{p}\end{array}$ & $\begin{array}{l}0.82^{p} \\
0.74^{p} \\
0.82^{p}\end{array}$ & $\begin{array}{l}5.0^{p} \\
4.4^{p} \\
4.5^{p}\end{array}$ & $\begin{array}{l}0.60^{p} \\
0.55^{p} \\
0.57^{p}\end{array}$ & $\begin{array}{l}3.6^{p} \\
3.0^{p} \\
3.1^{p}\end{array}$ & $\begin{array}{l}1.29^{p} \\
1.25^{p} \\
1.24^{p}\end{array}$ & $\begin{array}{l}7 . p^{p} \\
7.4^{p} \\
6.8^{p}\end{array}$ \\
\hline
\end{tabular}

Note: Death figures for England and Wales represent the number of deaths registered in each year up

Infant, neonatal and perinatal mortality rates for Northern Ireland have now been to 1992, and the number of deaths occurring in each year from 1993 to 2005. Death figures for amended to take account of the non-resident livebirths.

2006, 2007 and provisional death figures for 2008 relate to registrations.

The birth and death rates for 2008 are based on 2006-based population projections resident outside England and Wales. These events are, however, included in the totals for

for 2008 Math rates for 2008 are based on 2006-based population projections

England and Wales combined, and for the United Kingdom.
.

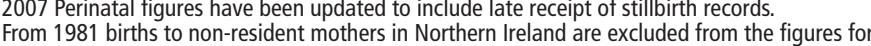
From 1981 births to non-resident mothers in No
Northern Ireland, and for the United Kingdom.

marital status estimates.
Provisional 2008 divorce statistics for Scotland have been withdrawn and will be released in spring 2009. 
Constituent countries of the United Kingdom

\begin{tabular}{|c|c|c|c|c|c|c|c|c|c|c|c|c|c|c|c|c|c|c|c|}
\hline \multirow{2}{*}{\multicolumn{2}{|c|}{$\begin{array}{l}\text { Year and } \\
\text { quarter }\end{array}$}} & \multicolumn{2}{|c|}{$\begin{array}{l}\text { All live } \\
\text { births }\end{array}$} & \multicolumn{2}{|c|}{$\begin{array}{c}\text { Live births } \\
\text { outside marriage }\end{array}$} & \multicolumn{2}{|c|}{ Marriages } & \multicolumn{2}{|c|}{$\begin{array}{c}\text { Civil } \\
\text { Partnerships }\end{array}$} & \multicolumn{2}{|c|}{ Divorces } & \multicolumn{2}{|c|}{ Deaths } & \multicolumn{2}{|c|}{$\begin{array}{c}\text { Infant } \\
\text { mortality }\end{array}$} & \multicolumn{2}{|c|}{$\begin{array}{l}\text { Neonatal } \\
\text { mortality }\end{array}$} & \multicolumn{2}{|c|}{$\begin{array}{l}\text { Perinatal } \\
\text { mortality }^{8}\end{array}$} \\
\hline & & Number & Rate $^{1}$ & Number & Rate $^{2}$ & Number & Rate $^{3}$ & Number & Rate $^{4}$ & Number & Rate $^{5}$ & Number & Rate $^{1}$ & Number & Rate $^{2}$ & Number & Rate $^{2}$ & Number & Rate $^{9}$ \\
\hline \multicolumn{2}{|c|}{$\begin{array}{l}\text { Wales } \\
1976 \\
1981 \\
1986 \\
1991 \\
1996\end{array}$} & $\begin{array}{l}33.4 \\
35.8 \\
37.0 \\
38.1 \\
34.9\end{array}$ & $\begin{array}{l}11.9 \\
12.7 \\
13.2 \\
13.3 \\
12.1\end{array}$ & $\begin{array}{r}2.9 \\
4.0 \\
7.8 \\
12.3 \\
14.4\end{array}$ & $\begin{array}{l}86 \\
112 \\
211 \\
323 \\
412\end{array}$ & $\begin{array}{l}19.5 \\
19.8 \\
19.5 \\
16.6 \\
14.8\end{array}$ & $\begin{array}{l}. . \\
. . \\
. . \\
. . \\
. .\end{array}$ & $\begin{array}{l}\vdots \\
\vdots \\
\vdots\end{array}$ & $\begin{array}{l}\vdots \\
\vdots \\
\vdots\end{array}$ & $\begin{array}{c}. . \\
7.8 \\
8.4 \\
8.4\end{array}$ & $\begin{array}{l}. . \\
. . \\
. . \\
. . \\
. .\end{array}$ & $\begin{array}{l}36.3 \\
35.0 \\
34.7 \\
34.1 \\
34.6\end{array}$ & $\begin{array}{l}13.0 \\
12.4 \\
12.3 \\
11.9 \\
12.0\end{array}$ & $\begin{array}{l}0.46 \\
0.45 \\
0.35 \\
0.25 \\
0.20\end{array}$ & $\begin{array}{r}13.7 \\
12.6 \\
9.5 \\
6.6 \\
5.6\end{array}$ & $\begin{array}{l}0.32 \\
0.29 \\
0.21 \\
0.16 \\
0.13\end{array}$ & $\begin{array}{l}9.6 \\
8.1 \\
5.6 \\
4.1 \\
3.6\end{array}$ & $\begin{array}{l}0.64 \\
0.51 \\
0.38 \\
0.30 \\
0.26\end{array}$ & $\begin{array}{r}19.0 \\
14.1 \\
10.3 \\
7.9 \\
7.5\end{array}$ \\
\hline $\begin{array}{l}1999 \\
2000 \\
2001 \\
2002 \\
2003\end{array}$ & & $\begin{array}{l}32.1 \\
31.3 \\
30.6 \\
30.2 \\
31.4\end{array}$ & $\begin{array}{l}11.1 \\
10.8 \\
10.5 \\
10.3 \\
10.7\end{array}$ & $\begin{array}{l}14.8 \\
14.8 \\
14.8 \\
15.0 \\
15.8\end{array}$ & $\begin{array}{l}461 \\
472 \\
483 \\
497 \\
503\end{array}$ & $\begin{array}{l}14.0 \\
14.1 \\
13.0 \\
13.5 \\
14.5\end{array}$ & $\begin{array}{l}. . \\
. . \\
. . \\
. . \\
. .\end{array}$ & $\begin{array}{l}\vdots \\
\vdots \\
\vdots\end{array}$ & $\begin{array}{l}\vdots \\
\vdots \\
\vdots\end{array}$ & $\begin{array}{l}7.5 \\
7.2 \\
7.4 \\
7.6 \\
7.7\end{array}$ & $\begin{array}{l}. . \\
. . \\
. . \\
. . \\
. .\end{array}$ & $\begin{array}{l}35.0 \\
33.3 \\
33.0 \\
33.2 \\
33.7\end{array}$ & $\begin{array}{l}12.1 \\
11.5 \\
11.3 \\
11.4 \\
11.5\end{array}$ & $\begin{array}{l}0.20 \\
0.17 \\
0.16 \\
0.14 \\
0.13\end{array}$ & $\begin{array}{l}6.1 \\
5.3 \\
5.4 \\
4.5 \\
4.3\end{array}$ & $\begin{array}{l}0.13 \\
0.11 \\
0.11 \\
0.10 \\
0.10\end{array}$ & $\begin{array}{l}4.0 \\
3.5 \\
3.5 \\
3.2 \\
3.1\end{array}$ & $\begin{array}{l}0.25 \\
0.23 \\
0.23 \\
0.24 \\
0.24\end{array}$ & $\begin{array}{l}7.7 \\
7.2 \\
7.5 \\
7.7 \\
7.6\end{array}$ \\
\hline $\begin{array}{l}2004 \\
2005 \\
2006 \\
2007\end{array}$ & & $\begin{array}{l}32.3 \\
32.6 \\
33.6 \\
34.4\end{array}$ & $\begin{array}{l}11.0 \\
11.0 \\
11.3 \\
11.5\end{array}$ & $\begin{array}{l}16.6 \\
17.1 \\
17.8 \\
18.5\end{array}$ & $\begin{array}{l}513 \\
524 \\
530 \\
538\end{array}$ & $\begin{array}{l}14.9^{2} \\
14.0 \\
13.5^{p} \\
12.8^{p}\end{array}$ & $\begin{array}{l}. . \\
. . \\
. . \\
. .\end{array}$ & $\begin{array}{l}0.07^{10} \\
0.56 \\
0.29\end{array}$ & $\begin{array}{l}: \\
. . \\
. . \\
. .\end{array}$ & $\begin{array}{l}7.9 \\
7.2 \\
6.9 \\
6.7^{p}\end{array}$ & $\begin{array}{l}. . \\
. . \\
. . \\
. .\end{array}$ & $\begin{array}{l}32.1 \\
32.1 \\
31.1 \\
32.1\end{array}$ & $\begin{array}{l}10.9 \\
10.9 \\
10.5 \\
10.8\end{array}$ & $\begin{array}{l}0.16 \\
0.13 \\
0.14 \\
0.18\end{array}$ & $\begin{array}{l}4.9 \\
4.1 \\
4.1 \\
5.3\end{array}$ & $\begin{array}{l}0.10 \\
0.09 \\
0.09 \\
0.12\end{array}$ & $\begin{array}{l}3.1 \\
2.9 \\
2.8 \\
3.4\end{array}$ & $\begin{array}{l}0.26 \\
0.24 \\
0.23 \\
0.25\end{array}$ & $\begin{array}{l}8.0 \\
7.4 \\
6.9 \\
7.3\end{array}$ \\
\hline 2006 & $\begin{array}{l}\text { March } \\
\text { June } \\
\text { Sept } \\
\text { Dec }\end{array}$ & $\begin{array}{l}8.1 \\
8.3 \\
8.8 \\
8.4\end{array}$ & $\begin{array}{l}11.1 \\
11.2 \\
11.8 \\
11.2\end{array}$ & $\begin{array}{l}4.2 \\
4.3 \\
4.8 \\
4.5\end{array}$ & $\begin{array}{l}520 \\
523 \\
543 \\
535\end{array}$ & $\begin{array}{l}1.4^{p} \\
3.8^{p} \\
6.1^{p} \\
2.2^{p}\end{array}$ & $\begin{array}{l}. . \\
. \\
. . \\
. .\end{array}$ & $\begin{array}{l}0.16 \\
0.15 \\
0.16 \\
0.09\end{array}$ & $\begin{array}{l}. . \\
. . \\
. . \\
. .\end{array}$ & $\begin{array}{l}1.8 \\
1.7 \\
1.7 \\
1.7\end{array}$ & $\begin{array}{l}. . \\
. . \\
. . \\
. .\end{array}$ & $\begin{array}{l}8.7 \\
7.6 \\
7.2 \\
7.5\end{array}$ & $\begin{array}{r}11.9 \\
10.3 \\
9.7 \\
10.1\end{array}$ & $\begin{array}{l}0.03 \\
0.03 \\
0.04 \\
0.04\end{array}$ & $\begin{array}{l}3.1 \\
4.1 \\
4.0 \\
5.1\end{array}$ & $\begin{array}{l}0.02 \\
0.02 \\
0.03 \\
0.03\end{array}$ & $\begin{array}{l}2.0 \\
2.4 \\
3.1 \\
3.6\end{array}$ & $\begin{array}{l}0.06 \\
0.05 \\
0.07 \\
0.06\end{array}$ & $\begin{array}{l}7.0 \\
6.3 \\
7.7 \\
6.6\end{array}$ \\
\hline 2007 & $\begin{array}{l}\text { March } \\
\text { June } \\
\text { Sept } \\
\text { Dec }\end{array}$ & $\begin{array}{l}8.1 \\
8.5 \\
9.1 \\
8.7\end{array}$ & $\begin{array}{l}11.0 \\
11.4 \\
12.1 \\
11.6\end{array}$ & $\begin{array}{l}4.3 \\
4.5 \\
4.9 \\
4.8\end{array}$ & $\begin{array}{l}536 \\
530 \\
541 \\
547\end{array}$ & $\begin{array}{l}1.3^{p} \\
3.6^{p} \\
5.7^{p} \\
2.2^{p}\end{array}$ & $\begin{array}{l}. . \\
. . \\
. . \\
. .\end{array}$ & $\begin{array}{l}0.06 \\
0.10 \\
0.08 \\
0.06\end{array}$ & $\begin{array}{l}. . \\
. . \\
. . \\
. .\end{array}$ & $\begin{array}{l}1.8^{\mathrm{p}} \\
1.8^{\mathrm{p}} \\
1.7^{\mathrm{p}} \\
1.4^{\mathrm{p}}\end{array}$ & $\begin{array}{l}. . \\
. . \\
. . \\
. .\end{array}$ & $\begin{array}{l}8.8 \\
7.9 \\
7.3 \\
8.3\end{array}$ & $\begin{array}{r}11.9^{\mathrm{P}} \\
10.6^{\mathrm{P}} \\
9.7^{\mathrm{P}} \\
11.0^{\mathrm{P}}\end{array}$ & $\begin{array}{l}0.05 \\
0.04 \\
0.04 \\
0.05\end{array}$ & $\begin{array}{l}6.3 \\
4.5 \\
4.4 \\
6.1\end{array}$ & $\begin{array}{l}0.03 \\
0.02 \\
0.03 \\
0.04\end{array}$ & $\begin{array}{l}3.7 \\
2.8 \\
2.8 \\
4.1\end{array}$ & $\begin{array}{l}0.07 \\
0.06 \\
0.05 \\
0.07\end{array}$ & $\begin{array}{l}9.0 \\
6.8 \\
5.7 \\
7.9\end{array}$ \\
\hline 2008 & $\begin{array}{l}\text { March } \\
\text { June } \\
\text { Sept }\end{array}$ & $\begin{array}{l}8.7^{p} \\
9.0^{p} \\
9.2^{p}\end{array}$ & $\begin{array}{l}11.8^{p} \\
12.0^{p} \\
12.2^{p}\end{array}$ & $\begin{array}{l}4.8^{p} \\
5.0^{p} \\
5.1^{p}\end{array}$ & $\begin{array}{l}552^{p} \\
555^{p} \\
556^{p}\end{array}$ & $\begin{array}{l}. . \\
. .\end{array}$ & $\begin{array}{l}. . \\
. . \\
. .\end{array}$ & $\begin{array}{l}0.06^{\mathrm{P}} \\
0.07^{\mathrm{P}} \\
. .\end{array}$ & $\begin{array}{l}. . \\
. . \\
. .\end{array}$ & $\begin{array}{l}1.6^{\mathrm{P}} \\
1.5^{\mathrm{P}} \\
. .\end{array}$ & $\begin{array}{l}. . \\
. .\end{array}$ & $\begin{array}{l}8.6^{p} \\
7.7^{p} \\
7.3^{p}\end{array}$ & $\begin{array}{r}11.5^{p} \\
10.4^{p} \\
9.6^{p}\end{array}$ & $\begin{array}{l}0.04^{p} \\
0.04^{p} \\
0.04^{p}\end{array}$ & $\begin{array}{l}4.1^{p} \\
4.3^{p} \\
3.8^{p}\end{array}$ & $\begin{array}{l}0.03^{p} \\
0.03^{p} \\
0.03^{p}\end{array}$ & $\begin{array}{l}3.0^{p} \\
3.3^{p} \\
2.9^{p}\end{array}$ & $\begin{array}{l}0.06^{\mathrm{p}} \\
0.06^{\mathrm{p}} \\
0.06^{\mathrm{P}}\end{array}$ & $\begin{array}{l}6.8^{p} \\
6.5^{p} \\
6.2^{p}\end{array}$ \\
\hline $\begin{array}{l}\text { Scotla } \\
1976 \\
1981 \\
1986 \\
1991 \\
1996\end{array}$ & & $\begin{array}{l}64.9 \\
69.1 \\
65.8 \\
67.0 \\
59.3\end{array}$ & $\begin{array}{l}12.5 \\
13.4 \\
12.9 \\
13.2 \\
11.6\end{array}$ & $\begin{array}{r}6.0 \\
8.5 \\
13.6 \\
19.5 \\
21.4\end{array}$ & $\begin{array}{r}93 \\
122 \\
206 \\
291 \\
360\end{array}$ & $\begin{array}{l}37.5 \\
36.2 \\
35.8 \\
33.8 \\
30.2\end{array}$ & $\begin{array}{l}53.8 \\
47.5 \\
42.9 \\
39.0 \\
33.2\end{array}$ & $\begin{array}{l}\vdots \\
\vdots \\
\vdots\end{array}$ & $\begin{array}{c}\vdots \\
\vdots \\
\vdots\end{array}$ & $\begin{array}{r}8.1 \\
9.9 \\
12.8 \\
12.4 \\
12.3\end{array}$ & $\begin{array}{r}6.5 \\
8.0 \\
10.7 \\
10.6 \\
10.9\end{array}$ & $\begin{array}{l}65.3 \\
63.8 \\
63.5 \\
61.0 \\
60.7\end{array}$ & $\begin{array}{l}12.5 \\
12.3 \\
12.4 \\
12.0 \\
11.9\end{array}$ & $\begin{array}{l}0.96 \\
0.78 \\
0.58 \\
0.47 \\
0.37\end{array}$ & $\begin{array}{r}14.8 \\
11.3 \\
8.8 \\
7.1 \\
6.2\end{array}$ & $\begin{array}{l}0.67 \\
0.47 \\
0.34 \\
0.29 \\
0.23\end{array}$ & $\begin{array}{r}10.3 \\
6.9 \\
5.2 \\
4.6 \\
3.9\end{array}$ & $\begin{array}{l}1.20 \\
0.81 \\
0.67 \\
0.58 \\
0.55\end{array}$ & $\begin{array}{r}18.3 \\
11.6 \\
10.2 \\
8.6 \\
9.2\end{array}$ \\
\hline $\begin{array}{l}1999 \\
2000 \\
2001 \\
2002 \\
2003\end{array}$ & & $\begin{array}{l}55.1 \\
53.1 \\
52.5 \\
51.3 \\
52.4\end{array}$ & $\begin{array}{l}10.9 \\
10.5 \\
10.4 \\
10.1 \\
10.4\end{array}$ & $\begin{array}{l}22.7 \\
22.6 \\
22.8 \\
22.5 \\
23.9\end{array}$ & $\begin{array}{l}412 \\
426 \\
433 \\
440 \\
455\end{array}$ & $\begin{array}{l}29.9 \\
30.4 \\
29.6 \\
29.8 \\
30.8\end{array}$ & $\begin{array}{l}31.5 \\
31.6 \\
31.0 \\
30.8 \\
31.3\end{array}$ & $\begin{array}{l}\vdots \\
\vdots \\
\vdots\end{array}$ & $\begin{array}{l}\vdots \\
\vdots \\
\vdots\end{array}$ & $\begin{array}{l}11.9 \\
11.1 \\
10.6 \\
10.8 \\
10.1\end{array}$ & $\begin{array}{r}10.9 \\
10.3 \\
9.7 \\
10.0 \\
10.2\end{array}$ & $\begin{array}{l}60.3 \\
57.8 \\
57.4 \\
58.1 \\
58.5\end{array}$ & $\begin{array}{l}11.9 \\
11.4 \\
11.3 \\
11.5 \\
11.6\end{array}$ & $\begin{array}{l}0.28 \\
0.31 \\
0.29 \\
0.27 \\
0.27\end{array}$ & $\begin{array}{l}5.0 \\
5.7 \\
5.5 \\
5.3 \\
5.1\end{array}$ & $\begin{array}{l}0.18 \\
0.21 \\
0.20 \\
0.16 \\
0.18\end{array}$ & $\begin{array}{l}3.3 \\
4.0 \\
3.8 \\
3.2 \\
3.4\end{array}$ & $\begin{array}{l}0.42 \\
0.45 \\
0.45 \\
0.39 \\
0.42\end{array}$ & $\begin{array}{l}7.6 \\
8.4 \\
8.5 \\
7.6 \\
8.0\end{array}$ \\
\hline $\begin{array}{l}2004 \\
2005 \\
2006 \\
2007\end{array}$ & & $\begin{array}{l}54.0 \\
54.4 \\
55.7 \\
57.8\end{array}$ & $\begin{array}{l}10.6 \\
10.7 \\
10.9 \\
11.2\end{array}$ & $\begin{array}{l}25.2 \\
25.6 \\
26.6 \\
28.4\end{array}$ & $\begin{array}{l}467 \\
471 \\
477 \\
491\end{array}$ & $\begin{array}{l}32.2 \\
30.9 \\
29.9 \\
29.9\end{array}$ & $\begin{array}{l}32.1 \\
30.3 \\
28.7 \\
28.1\end{array}$ & $\begin{array}{l}\vdots \\
0.08^{10} \\
1.05 \\
0.69\end{array}$ & $\begin{array}{l}\vdots \\
2.5^{10} \\
1.0 \\
0.6\end{array}$ & $\begin{array}{l}11.2 \\
10.9 \\
13.0 \\
12.8\end{array}$ & $\begin{array}{l}10.5 \\
10.3 \\
12.3 \\
12.2\end{array}$ & $\begin{array}{l}56.2 \\
55.7 \\
55.1 \\
56.0\end{array}$ & $\begin{array}{l}11.1 \\
11.0 \\
10.8 \\
10.9^{\mathrm{P}}\end{array}$ & $\begin{array}{l}0.27 \\
0.28 \\
0.25 \\
0.27\end{array}$ & $\begin{array}{l}4.9 \\
5.2 \\
4.5 \\
4.7\end{array}$ & $\begin{array}{l}0.17 \\
0.19 \\
0.17 \\
0.19\end{array}$ & $\begin{array}{l}3.1 \\
3.5 \\
3.1 \\
3.2\end{array}$ & $\begin{array}{l}0.44 \\
0.42 \\
0.42 \\
0.45\end{array}$ & $\begin{array}{l}8.1 \\
7.7 \\
7.4 \\
7.8\end{array}$ \\
\hline 2006 & $\begin{array}{l}\text { March } \\
\text { June } \\
\text { Sept } \\
\text { Dec }\end{array}$ & $\begin{array}{l}13.6 \\
14.0 \\
14.2 \\
13.9\end{array}$ & $\begin{array}{l}10.8 \\
11.0 \\
11.0 \\
10.8\end{array}$ & $\begin{array}{l}6.6 \\
6.7 \\
6.7 \\
6.6\end{array}$ & $\begin{array}{l}487 \\
475 \\
471 \\
477\end{array}$ & $\begin{array}{r}3.5 \\
8.3 \\
12.2 \\
5.9\end{array}$ & $\begin{array}{l}13.6 \\
32.1 \\
46.4 \\
22.4\end{array}$ & $\begin{array}{l}0.26 \\
0.32 \\
0.28 \\
0.19\end{array}$ & $\begin{array}{l}1.0 \\
1.2 \\
1.1 \\
0.7\end{array}$ & $\begin{array}{l}2.6 \\
3.1 \\
3.6 \\
3.7\end{array}$ & $\begin{array}{l}10.1 \\
11.7 \\
13.4 \\
14.1\end{array}$ & $\begin{array}{l}14.9 \\
13.9 \\
12.7 \\
13.6\end{array}$ & $\begin{array}{r}11.8 \\
10.9 \\
9.8 \\
10.6\end{array}$ & $\begin{array}{l}0.05 \\
0.07 \\
0.05 \\
0.07\end{array}$ & $\begin{array}{l}3.7 \\
5.0 \\
3.8 \\
5.3\end{array}$ & $\begin{array}{l}0.03 \\
0.05 \\
0.04 \\
0.04\end{array}$ & $\begin{array}{l}2.4 \\
3.3 \\
2.9 \\
3.7\end{array}$ & $\begin{array}{l}0.09 \\
0.09 \\
0.11 \\
0.12\end{array}$ & $\begin{array}{l}6.7 \\
6.4 \\
7.8 \\
8.7\end{array}$ \\
\hline
\end{tabular}

\begin{tabular}{|c|c|c|c|c|c|c|c|c|c|c|c|c|c|c|c|c|c|c|c|}
\hline & & & & & & & & & & & & & & & & & & & \\
\hline 2007 & $\begin{array}{l}\text { March } \\
\text { June } \\
\text { Sept } \\
\text { Dec }\end{array}$ & $\begin{array}{l}14.2 \\
14.3 \\
14.9 \\
14.4\end{array}$ & $\begin{array}{l}11.2 \\
11.1 \\
11.5 \\
11.1\end{array}$ & $\begin{array}{l}7.1 \\
6.9 \\
7.2 \\
7.1\end{array}$ & $\begin{array}{l}501 \\
482 \\
485 \\
497\end{array}$ & $\begin{array}{r}3.3 \\
8.1 \\
12.6 \\
5.8\end{array}$ & $\begin{array}{l}12.7 \\
30.7 \\
47.1 \\
21.6\end{array}$ & $\begin{array}{l}0.11 \\
0.18 \\
0.25 \\
0.15\end{array}$ & $\begin{array}{l}0.4 \\
0.7 \\
0.9 \\
0.6\end{array}$ & $\begin{array}{l}3.3 \\
3.3 \\
3.0 \\
3.1\end{array}$ & $\begin{array}{l}12.8 \\
12.7 \\
11.4 \\
11.7\end{array}$ & $\begin{array}{l}15.8 \\
13.4 \\
12.6 \\
14.2\end{array}$ & $\begin{array}{r}12.5 \\
10.4 \\
9.7 \\
10.9\end{array}$ & $\begin{array}{l}0.07 \\
0.08 \\
0.07 \\
0.06\end{array}$ & $\begin{array}{l}4.9 \\
5.3 \\
4.6 \\
3.9\end{array}$ & $\begin{array}{l}0.05 \\
0.05 \\
0.05 \\
0.04\end{array}$ & $\begin{array}{l}3.6 \\
3.4 \\
3.0 \\
2.9\end{array}$ & $\begin{array}{l}0.12 \\
0.12 \\
0.11 \\
0.11\end{array}$ & $\begin{array}{l}8.1 \\
8.6 \\
7.1 \\
7.5\end{array}$ \\
\hline 2008 & $\begin{array}{l}\text { March } \\
\text { June } \\
\text { Sept }\end{array}$ & $\begin{array}{l}15.1^{\mathrm{p}} \\
14.9^{\mathrm{p}} \\
15.5^{\mathrm{p}}\end{array}$ & $\begin{array}{l}11.8^{p} \\
11.6^{p} \\
12.1^{p}\end{array}$ & $\begin{array}{l}7.7^{p} \\
7.3^{p} \\
7.7^{p}\end{array}$ & $\begin{array}{l}510^{p} \\
488^{p} \\
498^{p}\end{array}$ & $\begin{array}{r}3.4^{\mathrm{p}} \\
7.9^{\mathrm{p}} \\
11.9^{\mathrm{p}}\end{array}$ & $\begin{array}{l}13.0^{p} \\
29.9^{p} \\
44.5^{p}\end{array}$ & $\begin{array}{l}0.10^{\mathrm{p}} \\
0.11^{\mathrm{P}} \\
0.20^{\mathrm{P}}\end{array}$ & $\begin{array}{l}0.4^{p} \\
0.4^{p} \\
0.7^{p}\end{array}$ & $\begin{array}{l}. . \\
. .\end{array}$ & $\begin{array}{l}. . \\
. .\end{array}$ & $\begin{array}{l}15.1^{p} \\
13.5^{p} \\
12.6^{p}\end{array}$ & $\begin{array}{r}11.8^{p} \\
10.5^{p} \\
9.7^{p}\end{array}$ & $\begin{array}{l}0.06^{p} \\
0.06^{p} \\
0.07^{p}\end{array}$ & $\begin{array}{l}3.9^{p} \\
4.0^{p} \\
4.8^{p}\end{array}$ & $\begin{array}{l}0.04^{p} \\
0.04^{p} \\
0.05^{p}\end{array}$ & $\begin{array}{l}2.8^{p} \\
2.5^{p} \\
3.4^{p}\end{array}$ & $\begin{array}{l}0.11^{\mathrm{P}} \\
0.11^{\mathrm{p}} \\
0.11^{\mathrm{p}}\end{array}$ & $\begin{array}{l}7.0^{p} \\
7.5^{p} \\
6.9^{p}\end{array}$ \\
\hline $\begin{array}{l}\text { North } \\
1976 \\
1981 \\
1986 \\
1991 \\
1996\end{array}$ & hern Ireland & $\begin{array}{l}26.4 \\
27.2 \\
28.0 \\
26.0 \\
24.4\end{array}$ & $\begin{array}{l}17.3 \\
17.6 \\
17.8 \\
16.2 \\
14.7\end{array}$ & $\begin{array}{l}1.3 \\
1.9 \\
3.6 \\
5.3 \\
6.3\end{array}$ & $\begin{array}{r}50 \\
70 \\
128 \\
203 \\
260\end{array}$ & $\begin{array}{r}9.9 \\
9.6 \\
10.2 \\
9.2 \\
8.3\end{array}$ & $\begin{array}{c}45.4 \\
. . \\
. . \\
. .\end{array}$ & $\begin{array}{l}\vdots \\
\vdots \\
\vdots\end{array}$ & $\begin{array}{l}\vdots \\
\vdots \\
\vdots\end{array}$ & $\begin{array}{l}0.6 \\
1.4 \\
1.5 \\
2.3 \\
2.3\end{array}$ & $\begin{array}{c}4.2 \\
. . \\
. . \\
. .\end{array}$ & $\begin{array}{l}17.0 \\
16.3 \\
16.1 \\
15.1 \\
15.2\end{array}$ & $\begin{array}{r}11.2 \\
10.6 \\
10.3 \\
9.4 \\
9.2\end{array}$ & $\begin{array}{l}0.48 \\
0.36 \\
0.36 \\
0.19 \\
0.14\end{array}$ & $\begin{array}{r}18.3 \\
13.2 \\
13.2 \\
7.4 \\
5.8\end{array}$ & $\begin{array}{l}0.35 \\
0.23 \\
0.23 \\
0.12 \\
0.09\end{array}$ & $\begin{array}{r}13.3 \\
8.3 \\
8.3 \\
4.6 \\
3.7\end{array}$ & $\begin{array}{l}0.59 \\
0.42 \\
0.42 \\
0.22 \\
0.23\end{array}$ & $\begin{array}{r}22.3 \\
15.3 \\
15.3 \\
8.4 \\
9.4\end{array}$ \\
\hline $\begin{array}{l}1999 \\
2000 \\
2001 \\
2002 \\
2003\end{array}$ & & $\begin{array}{l}23.0 \\
21.5 \\
22.0 \\
21.4 \\
21.6\end{array}$ & $\begin{array}{l}13.7 \\
12.8 \\
13.0 \\
12.6 \\
12.7\end{array}$ & $\begin{array}{l}7.0 \\
6.8 \\
7.1 \\
7.2 \\
7.4\end{array}$ & $\begin{array}{l}303 \\
318 \\
325 \\
335 \\
344\end{array}$ & $\begin{array}{l}7.6 \\
7.6 \\
7.3 \\
7.6 \\
7.8\end{array}$ & $\begin{array}{l}. . \\
. \\
. \\
. .\end{array}$ & $\begin{array}{c}\vdots \\
\vdots \\
\vdots\end{array}$ & $\begin{array}{l}\vdots \\
\vdots \\
\vdots\end{array}$ & $\begin{array}{l}2.3 \\
2.4 \\
2.4 \\
2.2 \\
2.3\end{array}$ & $\begin{array}{l}. . \\
. . \\
. . \\
. .\end{array}$ & $\begin{array}{l}15.7 \\
14.9 \\
14.5 \\
14.6 \\
14.5\end{array}$ & $\begin{array}{l}9.3 \\
8.9 \\
8.6 \\
8.6 \\
8.5\end{array}$ & $\begin{array}{l}0.15 \\
0.11 \\
0.13 \\
0.10 \\
0.12\end{array}$ & $\begin{array}{l}6.4 \\
5.0 \\
6.0 \\
4.6 \\
5.2\end{array}$ & $\begin{array}{l}0.11 \\
0.08 \\
0.10 \\
0.07 \\
0.09\end{array}$ & $\begin{array}{l}4.8 \\
3.8 \\
4.5 \\
3.5 \\
4.0\end{array}$ & $\begin{array}{l}0.23 \\
0.16 \\
0.19 \\
0.19 \\
0.18\end{array}$ & $\begin{array}{r}10.0 \\
7.2 \\
8.4 \\
8.7 \\
8.0\end{array}$ \\
\hline $\begin{array}{l}2004 \\
2005 \\
2006 \\
2007\end{array}$ & & $\begin{array}{l}22.3 \\
22.3 \\
23.3 \\
24.5\end{array}$ & $\begin{array}{l}13.0 \\
12.9 \\
13.4 \\
13.9\end{array}$ & $\begin{array}{l}7.7 \\
8.1 \\
8.8 \\
9.3\end{array}$ & $\begin{array}{l}345 \\
363 \\
380 \\
379\end{array}$ & $\begin{array}{l}8.3 \\
8.1 \\
8.3 \\
8.7\end{array}$ & $\begin{array}{l}. . \\
. . \\
. .\end{array}$ & $\begin{array}{l}\vdots \\
0.01^{10} \\
0.12^{\mathrm{p}} \\
0.11^{\mathrm{p}}\end{array}$ & $\begin{array}{l}: \\
. . \\
. . \\
. .\end{array}$ & $\begin{array}{l}2.5 \\
2.4 \\
2.6 \\
2.9^{p}\end{array}$ & $\begin{array}{l}. . \\
. . \\
. .\end{array}$ & $\begin{array}{l}14.4 \\
14.2 \\
14.5 \\
14.6\end{array}$ & $\begin{array}{l}8.4 \\
8.3 \\
8.4 \\
8.3\end{array}$ & $\begin{array}{l}0.12 \\
0.14 \\
0.12 \\
0.12\end{array}$ & $\begin{array}{l}5.5 \\
6.1 \\
5.1 \\
4.9\end{array}$ & $\begin{array}{l}0.08 \\
0.11 \\
0.09 \\
0.08\end{array}$ & $\begin{array}{l}3.7 \\
4.9 \\
3.8 \\
3.2\end{array}$ & $\begin{array}{l}0.19 \\
0.19 \\
0.17 \\
0.17\end{array}$ & $\begin{array}{l}8.0 \\
8.1 \\
6.9 \\
6.9\end{array}$ \\
\hline & $\begin{array}{l}\text { March } \\
\text { June } \\
\text { Sept } \\
\text { Dec }\end{array}$ & $\begin{array}{l}5.8 \\
5.8 \\
6.1 \\
5.6\end{array}$ & $\begin{array}{l}13.6 \\
13.3 \\
13.9 \\
12.8\end{array}$ & $\begin{array}{l}2.2 \\
2.2 \\
2.3 \\
2.2\end{array}$ & $\begin{array}{l}370 \\
381 \\
358 \\
393\end{array}$ & $\begin{array}{l}0.9 \\
2.3 \\
3.5 \\
1.5\end{array}$ & $\begin{array}{l}. . \\
. \\
. . \\
. .\end{array}$ & $\begin{array}{l}0.03 \\
0.04 \\
0.03 \\
0.02\end{array}$ & $\begin{array}{l}. . \\
. . \\
. .\end{array}$ & $\begin{array}{l}0.7 \\
0.7 \\
0.5 \\
0.6\end{array}$ & $\begin{array}{l}. . \\
. . \\
. .\end{array}$ & $\begin{array}{l}4.0 \\
3.6 \\
3.4 \\
3.5\end{array}$ & $\begin{array}{l}9.4 \\
8.4 \\
7.8 \\
7.9\end{array}$ & $\begin{array}{l}0.03 \\
0.03 \\
0.03 \\
0.03\end{array}$ & $\begin{array}{l}5.2 \\
4.6 \\
4.8 \\
5.8\end{array}$ & $\begin{array}{l}0.02 \\
0.02 \\
0.02 \\
0.03\end{array}$ & $\begin{array}{l}3.2 \\
3.6 \\
3.5 \\
4.9\end{array}$ & $\begin{array}{l}0.04 \\
0.04 \\
0.05 \\
0.04\end{array}$ & $\begin{array}{l}6.7 \\
7.3 \\
7.4 \\
6.3\end{array}$ \\
\hline 2007 & $\begin{array}{l}\text { March } \\
\text { June } \\
\text { Sept } \\
\text { Dec }\end{array}$ & $\begin{array}{l}6.1 \\
6.0 \\
6.5 \\
5.9\end{array}$ & $\begin{array}{l}14.2 \\
13.7 \\
14.5 \\
13.2\end{array}$ & $\begin{array}{l}2.4 \\
2.2 \\
2.5 \\
2.2\end{array}$ & $\begin{array}{l}383 \\
365 \\
386 \\
380\end{array}$ & $\begin{array}{l}1.0 \\
2.4 \\
3.8 \\
1.6\end{array}$ & $\begin{array}{l}. . \\
. \\
. .\end{array}$ & $\begin{array}{l}0.02 \\
0.03 \\
0.04 \\
0.02\end{array}$ & $\begin{array}{l}. . \\
. . \\
. .\end{array}$ & $\begin{array}{l}0.9 \\
0.7 \\
0.7 \\
0.6\end{array}$ & $\begin{array}{l}. . \\
. . \\
. .\end{array}$ & $\begin{array}{l}4.2 \\
3.6 \\
3.3 \\
3.6\end{array}$ & $\begin{array}{l}9.7 \\
8.2 \\
7.3 \\
8.1\end{array}$ & $\begin{array}{l}0.04 \\
0.03 \\
0.03 \\
0.02\end{array}$ & $\begin{array}{l}6.7 \\
5.1 \\
4.6 \\
3.3\end{array}$ & $\begin{array}{l}0.03 \\
0.02 \\
0.02 \\
0.01\end{array}$ & $\begin{array}{l}4.6 \\
2.9 \\
3.2 \\
2.2\end{array}$ & $\begin{array}{l}0.05 \\
0.04 \\
0.05 \\
0.04\end{array}$ & $\begin{array}{l}7.6 \\
6.7 \\
7.0 \\
6.5\end{array}$ \\
\hline 2008 & $\begin{array}{l}\text { March } \\
\text { June } \\
\text { Sept }\end{array}$ & $\begin{array}{l}6.5^{p} \\
6.3^{p} \\
6.5^{p}\end{array}$ & $\begin{array}{l}14.8^{p} \\
14.3^{p} \\
14.4^{p}\end{array}$ & $\begin{array}{l}2.4^{p} \\
2.4^{p} \\
2.5^{p}\end{array}$ & $\begin{array}{l}396^{p} \\
384^{p} \\
386^{p}\end{array}$ & $\begin{array}{l}1.1^{\mathrm{p}} \\
2.2^{\mathrm{p}} \\
3.6^{\mathrm{p}}\end{array}$ & $\begin{array}{l}. . \\
. .\end{array}$ & $\begin{array}{l}0.02^{p} \\
0.02^{p} \\
0.02^{p}\end{array}$ & $\begin{array}{l}. . \\
. .\end{array}$ & $\begin{array}{l}. . \\
. .\end{array}$ & $\begin{array}{l}. . \\
. .\end{array}$ & $\begin{array}{l}4.1^{p} \\
3.6^{p} \\
3.4^{p}\end{array}$ & $\begin{array}{l}9.4^{p} \\
8.2^{p} \\
7.7^{p}\end{array}$ & $\begin{array}{l}0.03^{\mathrm{P}} \\
0.03^{\mathrm{P}} \\
0.03^{\mathrm{P}}\end{array}$ & $\begin{array}{l}4.6^{p} \\
4.7^{p} \\
3.8^{p}\end{array}$ & $\begin{array}{l}0.03^{p} \\
0.02^{p} \\
0.02^{p}\end{array}$ & $\begin{array}{l}3.9^{p} \\
3.6^{p} \\
2.7^{p}\end{array}$ & $\begin{array}{l}0.05^{p} \\
0.04^{p} \\
0.05^{p}\end{array}$ & $\begin{array}{l}7.3^{p} \\
6.6^{p} \\
7.5^{p}\end{array}$ \\
\hline $\begin{array}{ll}1 & \mathrm{Pe} \\
2 & \mathrm{Pe} \\
3 & \mathrm{Pe} \\
4 & \mathrm{Pe} \\
5 & \mathrm{Pe} \\
6 & \mathrm{De}\end{array}$ & $\begin{array}{l}\text { ar 1,000 popul } \\
\text { ar 1,000 live b } \\
\text { arsons marryin } \\
\text { arsons forming } \\
\text { arsons divorci } \\
\text { eaths under } 1\end{array}$ & 1,0 & 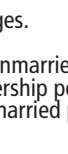 & & G. & & $e c$ & er. & & $\begin{array}{ll}7 & \text { Deatl } \\
8 & \text { Stillb } \\
9 & \text { Per } 1 \\
0 & \text { The C } \\
& \text { tables } \\
& \text { provis }\end{array}$ & $\begin{array}{l}\text { Part } \\
\text { al }\end{array}$ & n & 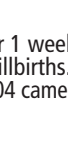 & 0 & & er & th & & \\
\hline
\end{tabular}




\begin{tabular}{|c|c|c|c|c|c|c|c|c|c|c|c|c|c|}
\hline \multirow[b]{2}{*}{$\begin{array}{l}\text { Year and } \\
\text { quarter }\end{array}$} & \multirow[b]{2}{*}{ Population } & \multirow[b]{2}{*}{$\begin{array}{l}\text { Live } \\
\text { births }\end{array}$} & \multirow[b]{2}{*}{ Deaths } & \multicolumn{2}{|c|}{ Dependency ratio } & \multicolumn{4}{|c|}{ Live births } & \multirow[b]{2}{*}{$\begin{array}{c}\text { Age- } \\
\text { standardised } \\
\text { mortality } \\
\text { rate }^{6}\end{array}$} & \multicolumn{2}{|c|}{$\begin{array}{l}\text { Period expectation of } \\
\text { life (in years) at birth }\end{array}$} & \\
\hline & & & & Children ${ }^{1}$ & Elderly $^{2}$ & $\mathrm{TFR}^{3}$ & $\begin{array}{c}\text { Standardised } \\
\text { mean age } \\
\text { of mother } \\
\text { at birth } \\
\text { (years) }^{4}\end{array}$ & $\begin{array}{l}\text { Unstand- } \\
\text { ardised } \\
\text { mean age of } \\
\text { mother at } \\
\text { birth (years) }\end{array}$ & $\begin{array}{c}\text { Outside } \\
\text { marriage as } \\
\text { percentage } \\
\text { of total } \\
\text { live births }\end{array}$ & & Males & Females & $\begin{array}{c}\text { Infant } \\
\text { mortality } \\
\text { rate }^{8}\end{array}$ \\
\hline
\end{tabular}

\section{United Kingdom}$$
1976 \text { Kingdom }
$$

$1981 \quad 56,216$.

$\begin{array}{ll}1986 & 56,683.8 \\ 1991 & 57,438\end{array}$

1996

$57,438.7$

\subsection{7}

754.8
792.3
733.2

680.8
658.0
660.7
646.2

658.0
660.7
646.2

42.1
37.1

33.5
33.2
33.9

$\begin{array}{ll}29.5 & 1.74 \\ 29.7 & 1.82 \\ 29.7 & 1.78 \\ 30.0 & 1.82\end{array}$

29.7
30.0
30.0

1.82
1.73

$\begin{array}{ll}2001 & 59,113.5 \\ 2002 & 59,323.5 \\ 2003 & 59,557.3 \\ 2004 & 59,845.8\end{array}$

669.1
668.8
695.6

602.3

716.0

606.2
612.0

32.6

32.2
31.8

29.8
29.8

29.9

31.4
31.0

29.9
30.0
30.0

1.63
1.64

1.64
1.71
1.77

583.1
582.7

2006

$60,587.3$
$60,975.4$

748.6
772.2

572.2
574.7

30.6
30.4

30.1
30.5

1.78

1.84
1.90

England

1976

1981
1986

$46,659.9 \quad 550.4$

$46,820.8$

$47,187.6$
$47,875.0$

550.4
598.2
623.6

623.6
660.8

560.3

$48,519.1$

614.2

541.0
544.5

544.5
534.0
524.0

41.4
36.4
33.1
32.9

29.7
29.9

29.8
30.0

1.70
1.79
1.76
1.81

$49,449.7$

563.7

2001
2002
2003

$49,652.3$

565.7
589.9

496.1
499.1

$50,110.7$

589.9
607.2

499.1
503.4

33.7

30.0

1.73

32.5
32.1
31.8

29.7
29.7
29.8

29.8
29.9

1.63
1.65
1.73

1.73
1.78

30.9

1.79

2006
2007

$50,762.9$
$51,092.0$

635.7
655.4

470.3

30.6
30.4

29.9
30.3

1.86
1.92

$2,799.3$

$2,813.5$
$2,810.9$

33.4
35.8
37.0
38.1
34.9

$\begin{array}{ll}36.3 & 42.0 \\ 35.0 & 37.6 \\ 34.7 & 34.3 \\ 34.1 & 34.4\end{array}$

30.9
31.6
32.5
33.5
33.7

1.78
1.87
1.86
1.88
1.81

$2,891.3$

30.6
30.2
31.4

$2,910.2$

2,919.8

$2,931.1$

31.4
32.3
32.6

33.0
33.2
33.7
32.1
32.1

33.7

33.3
32.8
32.3

33.6
33.7
33.8
33.9
34.1

1.66

1.64
1.73
1.78

1.78
1.81

$2,965.9 \quad 33.6$

2006

Scotland

1976

1981
1986

1991

$5,233.4$
$5,180.2$

$5,111.8$

$5,083.3$
$5,092.2$

64.9

69.1
65.8

65.8
67.0
59.3

31.1

31.4

34.3

1.86

1.86
1.90

$65.3 \quad 44.7$

63.8
63.5
61.0

63.5
61.0
60.7

44.7
38.2
33.6
32.4
32.3

28.4
28.4
28.1

28.1
28.9
29.2

1.79
1.84
1.67

1.67
1.69
1.56

2001
2002
2003

$5,064.2$

$5,054.8$

52.5
55.3
52.4
5

57.4
58.1
5

58.1
58.5
56.2
55.7

30.8
30.3

30.3
29.9

30.0

30.2
30.3
30.5
30.6

1.49
1.48

1.48
1.54
1.60

1.60

$5,094.8$

54.4

30.6
31.0

1.67
1.73

5,144.2

55.7
57.8

55.1
56.0

28.7
28.4

26.4

27.2
28.0

$\begin{array}{ll}17.0 & 56.1 \\ 16.3 & 50.6\end{array}$

16.1

15.1

56.1
50.6
46.1
44.1

25.3
25.3
25.5
26.1

25.5

2.68
2.59
2.45
2.16

2.16
1.95

38.6

$\begin{array}{ll}14.5 & 38.6 \\ 14.6 & 37.9\end{array}$

$\begin{array}{ll}14.6 & 37.9 \\ 14.4 & 36.4\end{array}$

25.5
25.7

25.9
26.2

1.80

1.77
1.81
1.87

1.87

$\begin{array}{ll}14.5 & 35.3 \\ 14.6 & 34.9\end{array}$

26.4
26.6

1.94
2.02

$\begin{array}{ll}2006 & 1,741.6 \\ 2007 & 1,759.1\end{array}$

23.3
24.5

Note: Death figures for England and Wales represent the number of deaths registered in each year up to 1992, and the number of deaths occurring in each year from 1993 to 2005. Dear up to 1992, and the number of deaths occurring in

Death figures for 2006 onwards relate to registrations.

列 usually resident outside England and Wales (apart from life expectancy figures - wher those occurring in England and Wales are assigned to England). These events are,

however, included in the total for the United Kingdom. From 1981 births to non-
resident mothers in Northern Ireland are excluded from the figures for Northern Ireland and for the United Kingdom.

1 Percentage of children under 16 to working-age population (males 16-64 and females 16-59).

2 Percentage of males 65 and over and females 60 and over to working-age population

(males 16-64 and females 16-59).

$\begin{array}{rrrrrrr}26.7 & 26.4 & 9.0 & 10,486 & & & \\ 27.0 & 26.8 & 12.5 & 9,506 & 70.8 & 76.8 & 14.5 \\ 27.4 & 27.0 & 20.4 & 8,914 & 71.9 & 77.7 & 9.5 \\ 27.7 & 27.7 & 29.8 & 8,168 & 73.2 & 78.7 & 7.4 \\ 28.2 & 28.6 & 35.5 & 7,584 & 74.2 & 79.4 & 6.1 \\ 28.6 & 29.2 & 40.1 & 6,807 & 75.6 & 80.4 & 5.5 \\ 28.7 & 29.3 & 40.6 & 6,765 & 75.9 & 80.5 & 5.2 \\ 28.8 & 29.4 & 41.5 & 6,758 & 76.2 & 80.7 & 5.3 \\ 28.9 & 29.4 & 42.3 & 6,394 & 76.5 & 80.9 & 5.0 \\ 29.1 & 29.5 & 42.9 & 6,268 & 76.9 & 81.3 & 5.1 \\ 29.1 & 29.5 & 43.7 & 6,067 & 77.2 & 81.5 & 5.0 \\ 29.3 & 29.5 & 44.4 & 5,966 & . . & . . & 4.7\end{array}$

$\begin{array}{rrrrrrr}26.5 & 26.4 & 9.2 & 10,271 & & & \\ 27.0 & 26.8 & 12.9 & 9.298 & 71.1 & 77.0 & 10.9 \\ 27.4 & 27.0 & 21.4 & 8,775 & 72.2 & 77.99 & 9.5 \\ 27.7 & 27.7 & 30.1 & 8,017 & 73.4 & 78.9 & 7.3 \\ 28.2 & 28.7 & 35.5 & 7,414 & 74.5 & 79.6 & 6.1 \\ 28.6 & 29.3 & 39.6 & 6,650 & 75.9 & 80.6 & 5.4 \\ 28.7 & 29.4 & 40.1 & 6,603 & 76.1 & 80.7 & 5.2 \\ 28.9 & 29.4 & 40.9 & 6,602 & 76.5 & 80.9 & 5.3 \\ 29.0 & 29.5 & 41.7 & 6,232 & 76.8 & 81.1 & 5.0 \\ 29.1 & 29.5 & 42.3 & 6,110 & 77.2 & 81.5 & 5.0 \\ 29.2 & 29.5 & 43.0 & 5,916 & 77.5 & 81.7 & 5.0 \\ 29.3 & 29.6 & 43.8 & 5.792 & & & 4.8\end{array}$

$26.2 \quad 26.0$

$\begin{array}{ll}26.7 & 26.6 \\ 26.9 & 26.5 \\ 27.1 & 27.0\end{array}$

8.6
11.2
21.1
32.3
41.2

10,858

9,846
9,043
8,149

$\begin{array}{ll}70.4 & 76 . .4 \\ 71.6 & 77.5 \\ 73.1 & 78.8 \\ 73.8 & 79.1\end{array}$

13.7
12.6

27.8

28.0
28.1
28.2

28.3
28.4
28.5

28.5
28.5
28.5

48.3
49.7
55.3
51.3
52.4

7,017

$75.3 \quad 80.0$

$\begin{array}{ll}75.5 & 80.1 \\ 75.8 & 80.3 \\ 76.1 & 80.6\end{array}$

6,984
6,588

76.1

80.9

76.7

81.1

6,307

$\begin{array}{rrr} & & \\ 69.1 & 75.3 & 14.8 \\ 70.2 & 76.2 & 8.3 \\ 71.4 & 77.1 & 7.1 \\ 72.2 & 77.9 & 6.2\end{array}$

$26.4 \quad 26.0$

$\begin{array}{ll}26.8 & 26.3 \\ 27.1 & 26.6 \\ 27.5 & 27.4\end{array}$

9.3
12.2
20.6
29.1
36.0

11,675
10,849

10,849
10,120

10,120
9,216
8,791

28.5

28.7

28.9

29.2

29.3

29.4

43.3
44.0
45.5

45.5
46.7
47.1

7,930
7,955

7,955
7,921
7,536

7,536

$\begin{array}{ll}73.3 & 78.8 \\ 73.5 & 78.9 \\ 73.8 & 79.1\end{array}$

$\begin{array}{ll}73.5 & 78.9 \\ 73.8 & 79.1 \\ 74.2 & 79.3 \\ 74.6 & 79.6\end{array}$

$74.6 \quad 79.6$

5.5
5.1
4.9

29.1
29.2

29.5

47.7
49.1

7,161
7,150

74.8

79.7

4.5
4.7

$\begin{array}{ll}27.8 & 27.4 \\ 28.1 & 27.5 \\ 28.1 & 27.5 \\ 28.3 & 28.0\end{array}$

$28.3 \quad 28.0$

5.0
7.0
12.8
20.3
26.0

11,746
10,567
10,071
8,303

8,303
7,742

69.2
70.9

75.5
77.1
78.4

18.3
13.2
13.2

$\begin{array}{llll}26.0 & 73.8 & 79.2 & 5.8\end{array}$

29.129 .4

29.2

29.5

32.5
33.5
34.4
34.5
36.3

$\begin{array}{ll}29.5 & 34.4 \\ 29.7 & 34.5\end{array}$

6,976
6,930

6,930
6,743
6,609

6,609

$\begin{array}{ll}75.2 & 80.1 \\ 75.6 & 80.4\end{array}$

$\begin{array}{ll}75.6 & 80.4 \\ 75.8 & 80.6\end{array}$

$\begin{array}{ll}76.0 & 80.8 \\ 76.1 & 81.0\end{array}$

6.1
4.7

4.7
5.3
5.5

$\begin{array}{llll}29.6 & 29.7 & 38.0 & 6,397 \\ 29.8 & 29.8 & 37.9 & 6,321\end{array}$

$76.2 \quad 81.2$

5.1
4.9

3 TFR (total fertility rate) is the number of children that would be born to a woman if current patterns of fertility persisted throughout her childbearing life. It is sometimes called the TPFR (total period fertility rate).

4 Standardised to take account of the age structure of the population.

5 Unstandardised and therefore takes no account of the age structure of the population.

6 Per million population. The age-standardised mortality rate makes allowances for changes in the age structure of the population. See Notes to tables.

7 All countries: figures for all years based on registered deaths. A minor methodological change was introduced for the 2006 figures to ensure consistency with population estimates of the very elderly for England and Wales. The effect on calculated life 


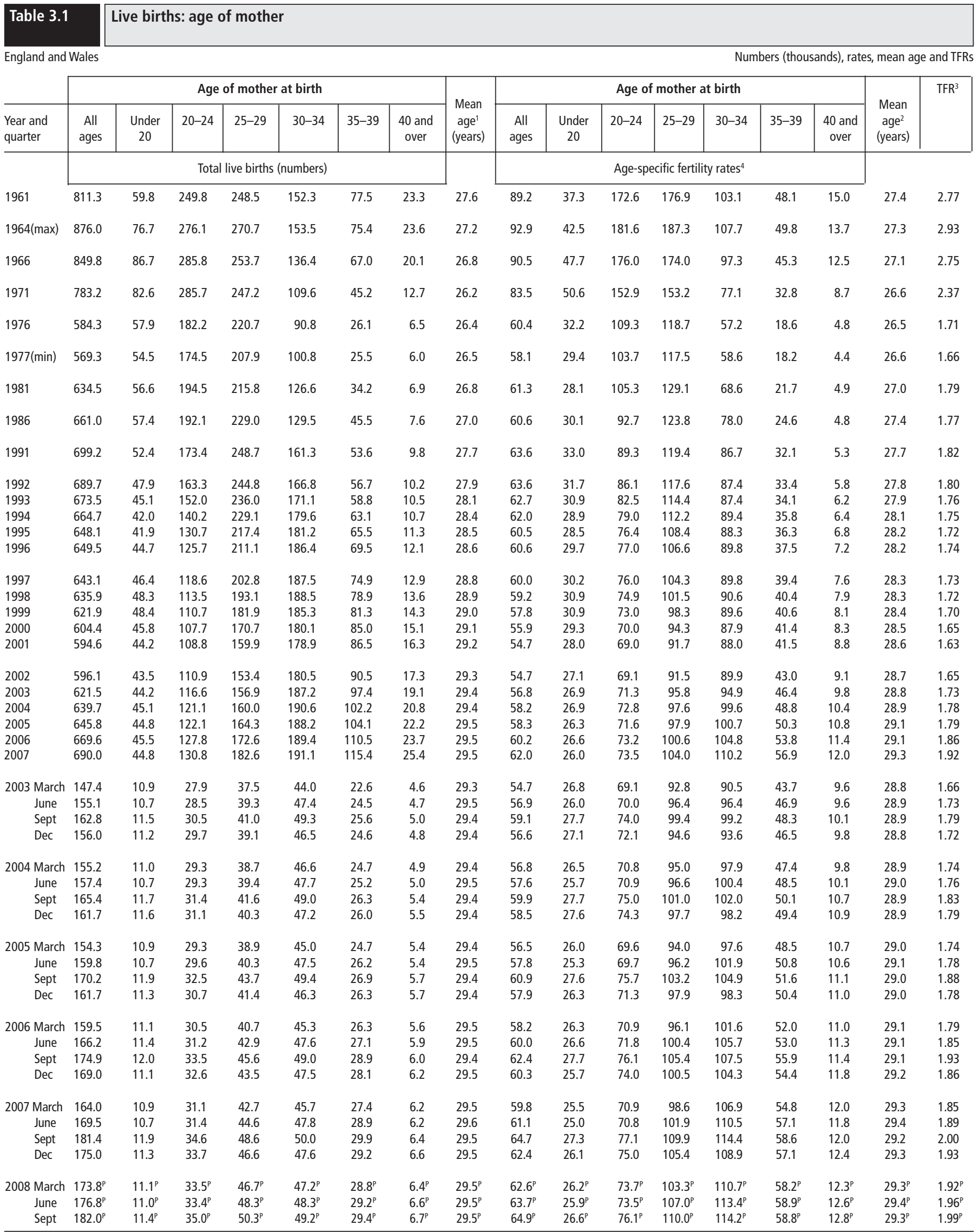

Note: The rates for women of all ages, under 20, and 40 and over are based upon the populations of women aged 15-44, 15-19, and 40-44 respectively. Rates for 2008 are based on 2006-based population projections for 2008.

1 Unstandardised and therefore takes no account of the age structure of the population.

2 Standardised to take account of the age structure of the population. This measure is more appropriate for use when analysing trends or making comparisons between different geographies.

3 TFR (total fertility rate) is the number of children that would be born to a woman if current patterns of fertility persisted throughout her childbearing life. It is sometimes called the TPFR (total period fertility rate).

4 Births per 1,000 women in the age-group; all quarterly age-specific fertility rates are adjusted for days in the quarter. They are not adjusted for seasonality.

p provisional. 
Numbers (thousands), mean age and percentages

\begin{tabular}{|c|c|c|c|c|c|c|c|c|c|c|c|c|c|c|c|c|c|c|}
\hline \multirow{3}{*}{$\begin{array}{l}\text { Year and } \\
\text { quarter }\end{array}$} & \multicolumn{7}{|c|}{ Age of mother at birth } & & \multicolumn{7}{|c|}{ Age of mother at birth } & \multicolumn{3}{|c|}{ Registration ${ }^{2}$} \\
\hline & All & Under & $20-24$ & $25-29$ & $30-34$ & $35-39$ & 40 and & \multirow{3}{*}{$\begin{array}{c}\text { Mean } \\
\text { age }^{1} \\
\text { (years) }\end{array}$} & \multirow{2}{*}{$\begin{array}{c}\text { All } \\
\text { ages }\end{array}$} & \multirow{2}{*}{$\begin{array}{l}\text { Under } \\
20\end{array}$} & \multirow[t]{2}{*}{$20-24$} & \multirow[t]{2}{*}{$25-29$} & \multirow[t]{2}{*}{$30-34$} & \multirow[t]{2}{*}{$35-39$} & \multirow{2}{*}{$\begin{array}{c}40 \text { and } \\
\text { over }\end{array}$} & \multicolumn{2}{|c|}{ Joint } & \multirow[t]{2}{*}{ Sole } \\
\hline & & & & & & & & & & & & & & & & $\begin{array}{l}\text { Same }^{3} \\
\text { address }\end{array}$ & $\begin{array}{c}\text { Different }{ }^{3} \\
\text { address }\end{array}$ & \\
\hline & \multicolumn{7}{|c|}{ Live births outside marriage (numbers) } & & \multicolumn{7}{|c|}{$\begin{array}{l}\text { Percentage of total live births } \\
\text { in age group }\end{array}$} & \multicolumn{3}{|c|}{$\begin{array}{l}\text { As a percentage of all } \\
\text { births outside marriage }\end{array}$} \\
\hline
\end{tabular}

\begin{tabular}{|c|c|c|c|c|c|c|c|c|c|c|c|c|c|c|c|c|c|c|c|}
\hline 1971 & & 65.7 & 21.6 & 22.0 & 11.5 & 6.2 & 3.2 & 1.1 & 23.7 & 8.4 & 26.1 & 7.7 & 4.7 & 5.7 & 7.0 & 9.0 & \multicolumn{2}{|c|}{45.5} & 54.5 \\
\hline 1976 & & 53.8 & 19.8 & 16.6 & 9.7 & 4.7 & 2.3 & 0.7 & 23.3 & 9.2 & 34.2 & 9.1 & 4.4 & 5.2 & 8.6 & 10.1 & \multirow{2}{*}{\multicolumn{2}{|c|}{$\begin{array}{l}51.0 \\
58.2\end{array}$}} & 49.0 \\
\hline 1981 & & 81.0 & 26.4 & 28.8 & 14.3 & 7.9 & 1.3 & 0.9 & 23.4 & 12.8 & 46.7 & 14.8 & 6.6 & 6.2 & 3.9 & 12.5 & & & 41.8 \\
\hline 1986 & & 141.3 & 39.6 & 54.1 & 27.7 & 13.1 & 5.7 & 1.1 & 23.8 & 21.4 & 69.0 & 28.2 & 12.1 & 10.1 & 12.6 & 14.7 & 46.6 & 19.6 & 33.8 \\
\hline 1991 & & 211.3 & 43.4 & 77.8 & 52.4 & 25.7 & 9.8 & 2.1 & 24.8 & 30.2 & 82.9 & 44.9 & 21.1 & 16.0 & 18.3 & 21.3 & 54.6 & 19.8 & 25.6 \\
\hline 1992 & & 215.2 & 40.1 & 77.1 & 55.9 & 28.9 & 10.9 & 2.3 & 25.2 & 31.2 & 83.7 & 47.2 & 22.8 & 17.3 & 19.3 & 22.9 & 55.4 & 20.7 & 23.9 \\
\hline 1993 & & 216.5 & 38.2 & 75.0 & 57.5 & 31.4 & 11.9 & 2.5 & 25.5 & 32.2 & 84.8 & 49.4 & 24.4 & 18.4 & 20.2 & 23.5 & 54.8 & 22.0 & 23.2 \\
\hline 1994 & & 215.5 & 35.9 & 71.0 & 58.5 & 34.0 & 13.4 & 2.7 & 25.8 & 32.4 & 85.5 & 50.6 & 25.5 & 18.9 & 21.2 & 25.2 & 57.5 & 19.8 & 22.7 \\
\hline 1995 & & 219.9 & 36.3 & 69.7 & 59.6 & 37.0 & 14.4 & 3.0 & 26.0 & 33.9 & 86.6 & 53.3 & 27.4 & 20.4 & 22.0 & 26.2 & 58.1 & 20.1 & 21.8 \\
\hline 1996 & & 232.7 & 39.3 & 71.1 & 62.3 & 40.5 & 16.2 & 3.2 & 26.1 & 35.8 & 88.0 & 56.5 & 29.5 & 21.7 & 23.4 & 26.7 & 58.1 & 19.9 & 21.9 \\
\hline 1997 & & 238.2 & 41.1 & 69.5 & 63.4 & 42.2 & 18.2 & 3.7 & 26.2 & 37.0 & 88.7 & 58.6 & 31.3 & 22.5 & 24.3 & 28.6 & 59.5 & 19.3 & 21.2 \\
\hline 1998 & & 240.6 & 43.0 & 67.8 & 62.4 & 43.9 & 19.6 & 3.9 & 26.3 & 37.8 & 89.1 & 59.7 & 32.3 & 23.3 & 24.8 & 29.0 & 60.9 & 18.3 & 20.8 \\
\hline 1999 & & 241.9 & 43.0 & 67.5 & 61.2 & 45.0 & 20.8 & 4.3 & 26.4 & 38.9 & 89.0 & 61.0 & 33.6 & 24.3 & 25.6 & 30.2 & 61.8 & 18.2 & 19.9 \\
\hline 2000 & & 238.6 & 41.1 & 67.5 & 59.1 & 43.9 & 22.3 & 4.7 & 26.5 & 39.5 & 89.7 & 62.6 & 34.6 & 24.4 & 26.2 & 31.0 & 62.7 & 18.2 & 19.2 \\
\hline 2001 & & 238.1 & 39.5 & 68.1 & 56.8 & 45.2 & 23.3 & 5.1 & 26.7 & 40.0 & 89.5 & 62.6 & 35.5 & 25.3 & 26.9 & 31.6 & 63.2 & 18.4 & 18.4 \\
\hline 2002 & & 242.0 & 38.9 & 70.2 & 55.8 & 46.4 & 25.1 & 5.6 & 26.8 & 40.6 & 89.5 & 63.3 & 36.4 & 25.7 & 27.7 & 32.2 & 63.7 & 18.5 & 17.8 \\
\hline 2003 & & 257.2 & 39.9 & 75.7 & 58.2 & 49.2 & 27.8 & 6.4 & 26.9 & 41.4 & 90.2 & 64.9 & 37.1 & 26.3 & 28.5 & 33.3 & 63.5 & 19.0 & 17.4 \\
\hline 2004 & & 269.7 & 41.0 & 79.8 & 61.4 & 50.7 & 29.7 & 7.1 & 27.0 & 42.2 & 91.0 & 65.9 & 38.4 & 26.6 & 29.0 & 34.0 & 63.6 & 19.6 & 16.8 \\
\hline 2005 & & 276.5 & 41.2 & 82.1 & 64.4 & 50.8 & 30.3 & 7.7 & 27.0 & 42.8 & 91.8 & 67.2 & 39.2 & 27.0 & 29.1 & 34.8 & 63.5 & 20.2 & 16.3 \\
\hline 2006 & & 291.4 & 42.3 & 87.7 & 69.3 & 51.4 & 32.2 & 8.4 & 27.0 & 43.5 & 93.0 & 68.6 & 40.1 & 27.1 & 29.2 & 35.5 & 63.7 & 20.8 & 15.6 \\
\hline 2007 & & 305.6 & 41.7 & 91.9 & 76.0 & 53.0 & 34.0 & 9.0 & 27.1 & 44.3 & 93.1 & 70.3 & 41.6 & 27.7 & 29.5 & 35.5 & 65.0 & 20.1 & 15.0 \\
\hline 2002 & March & 58.0 & 9.4 & 16.7 & 13.6 & 10.9 & 6.0 & 1.3 & 26.8 & 40.5 & 89.4 & 63.0 & 36.4 & 25.4 & 27.7 & 31.5 & 63.2 & 18.5 & 18.3 \\
\hline & June & 58.3 & 9.3 & 16.6 & 13.5 & 11.4 & 6.1 & 1.4 & 26.8 & 39.6 & 89.4 & 62.2 & 35.6 & 25.0 & 27.2 & 31.7 & 64.2 & 18.2 & 17.7 \\
\hline & Sept & 63.4 & 10.2 & 18.4 & 14.6 & 12.3 & 6.5 & 1.5 & 26.8 & 40.9 & 89.3 & 63.8 & 36.6 & 26.1 & 27.9 & 32.7 & 63.9 & 18.5 & 17.5 \\
\hline & Dec & 62.3 & 10.0 & 18.4 & 14.1 & 11.9 & 6.5 & 1.5 & 26.8 & 41.4 & 89.7 & 64.1 & 36.9 & 26.4 & 28.0 & 32.8 & 63.3 & 18.9 & 17.8 \\
\hline 2003 & March & 61.0 & 9.8 & 18.0 & 13.9 & 11.6 & 6.3 & 1.5 & 26.8 & 41.4 & 90.1 & 64.5 & 37.0 & 26.9 & 29.1 & 33.3 & 63.0 & 18.9 & 18.1 \\
\hline & June & 62.8 & 9.6 & 18.3 & 14.2 & 12.2 & 6.9 & 1.6 & 27.0 & 40.5 & 90.0 & 64.0 & 36.2 & 25.7 & 28.3 & 33.7 & 64.0 & 18.5 & 17.4 \\
\hline & Sept & 67.6 & 10.3 & 20.0 & 15.3 & 13.0 & 7.3 & 1.7 & 26.9 & 41.5 & 90.2 & 65.6 & 38.3 & 26.4 & 28.6 & 33.3 & 63.7 & 19.3 & 18.0 \\
\hline & Dec & 65.8 & 10.2 & 19.5 & 14.9 & 12.5 & 7.3 & 1.6 & 26.9 & 42.2 & 90.4 & 65.6 & 38.0 & 27.7 & 29.5 & 32.9 & 63.3 & 19.4 & 17.4 \\
\hline 2004 & March & 65.2 & 10.1 & 19.3 & 14.8 & 12.5 & 7.0 & 1.7 & 26.9 & 42.0 & 91.2 & 65.8 & 38.2 & 26.8 & 28.2 & 34.3 & 63.1 & 19.4 & 17.4 \\
\hline & June & 65.2 & 9.8 & 19.1 & 14.9 & 12.5 & 7.3 & 1.7 & 27.0 & 41.4 & 91.0 & 65.1 & 37.7 & 26.2 & 28.8 & 34.5 & 63.9 & 19.5 & 16.6 \\
\hline & Sept & 70.2 & 10.7 & 20.7 & 16.1 & 13.0 & 7.9 & 1.8 & 27.0 & 42.4 & 91.2 & 66.1 & 38.6 & 26.5 & 30.0 & 33.5 & 63.7 & 19.7 & 16.6 \\
\hline & Dec & 69.1 & 10.6 & 20.7 & 15.7 & 12.7 & 7.5 & 1.9 & 26.9 & 42.7 & 90.6 & 66.6 & 39.0 & 27.0 & 29.0 & 33.9 & 63.6 & 19.8 & 16.6 \\
\hline 2005 & March & 66.3 & 10.1 & 19.6 & 15.2 & 12.2 & 7.3 & 1.9 & 27.0 & 43.0 & 92.0 & 67.0 & 39.0 & 27.1 & 29.6 & 35.2 & 63.1 & 20.3 & 16.6 \\
\hline & June & 66.6 & 9.8 & 19.7 & 15.4 & 12.5 & 7.4 & 1.8 & 27.0 & 41.7 & 91.2 & 66.5 & 38.2 & 26.4 & 28.1 & 33.5 & 63.7 & 19.8 & 16.5 \\
\hline & Sept & 73.7 & 10.9 & 22.1 & 17.3 & 13.4 & 7.9 & 2.1 & 26.9 & 43.3 & 92.0 & 68.0 & 39.6 & 27.2 & 29.3 & 35.7 & 63.7 & 20.3 & 16.0 \\
\hline & Dec & 69.9 & 10.4 & 20.7 & 16.5 & 12.6 & 7.7 & 2.0 & 27.0 & 43.2 & 92.1 & 67.4 & 39.8 & 27.3 & 29.5 & 34.8 & 63.5 & 20.3 & 16.2 \\
\hline 2006 & March & 68.7 & 10.3 & 20.8 & 16.0 & 12.0 & 7.6 & 1.9 & 26.9 & 43.1 & 93.1 & 68.1 & 39.4 & 26.5 & 28.9 & 34.4 & 63.1 & 20.9 & 16.0 \\
\hline & June & 71.4 & 10.5 & 21.2 & 16.9 & 12.8 & 7.8 & 2.1 & 27.0 & 43.0 & 92.6 & 68.0 & 39.4 & 26.9 & 28.8 & 35.0 & 63.7 & 20.6 & 15.6 \\
\hline & Sept & 76.8 & 11.1 & 23.1 & 18.6 & 13.4 & 8.4 & 2.2 & 27.0 & 43.9 & 92.8 & 69.0 & 40.7 & 27.3 & 29.2 & 36.9 & 64.1 & 20.5 & 15.4 \\
\hline & Dec & 74.5 & 10.3 & 22.6 & 17.8 & 13.2 & 8.4 & 2.2 & 27.1 & 44.1 & 93.3 & 69.2 & 40.9 & 27.8 & 29.8 & 35.7 & 63.6 & 21.0 & 15.4 \\
\hline 2007 & March & 72.5 & 10.2 & 21.7 & 17.6 & 12.6 & 8.2 & 2.2 & 27.1 & 44.2 & 93.5 & 69.8 & 41.3 & 27.5 & 29.8 & 35.1 & 64.0 & 20.5 & 15.5 \\
\hline & June & 73.5 & 9.9 & 21.8 & 18.3 & 13.0 & 8.3 & 2.2 & 27.1 & 43.4 & 92.6 & 69.5 & 41.0 & 27.2 & 28.8 & 35.2 & 65.1 & 19.9 & 14.9 \\
\hline & Sept & 80.8 & 11.1 & 24.4 & 20.4 & 13.9 & 8.8 & 2.2 & 27.0 & 44.5 & 93.2 & 70.5 & 41.9 & 27.8 & 29.6 & 35.0 & 65.2 & 20.1 & 14.7 \\
\hline & Dec & 78.7 & 10.6 & 24.0 & 19.7 & 13.5 & 8.7 & 2.4 & 27.1 & 45.0 & 93.1 & 71.3 & 42.2 & 28.3 & 29.6 & 36.5 & 65.3 & 19.9 & 14.8 \\
\hline 2008 & March & $78.5^{p}$ & $10.5^{P}$ & $23.8^{\mathrm{p}}$ & $20.0^{p}$ & $13.3^{p}$ & $8.6^{p}$ & $2.3^{p}$ & $27.1^{\mathrm{P}}$ & $45.2^{p}$ & $94.3^{p}$ & $71.1^{\mathrm{p}}$ & $42.8^{p}$ & $28.3^{P}$ & $29.9^{p}$ & $35.8^{p}$ & $65.3^{P}$ & $20.0^{\mathrm{P}}$ & $14.7^{P}$ \\
\hline & June & $79.3^{P}$ & $10.3^{P}$ & $23.9^{p}$ & $20.5^{p}$ & $13.7^{\mathrm{P}}$ & $8.6^{P}$ & $2.4^{\mathrm{P}}$ & $27.1^{p}$ & $44.9^{p}$ & $93.5^{\mathrm{P}}$ & $71.4^{\mathrm{P}}$ & $42.4^{\mathrm{P}}$ & $28.4^{\mathrm{P}}$ & $29.5^{\mathrm{P}}$ & $35.9^{\mathrm{p}}$ & $66.0^{p}$ & $19.8^{\mathrm{p}}$ & $14.2^{P}$ \\
\hline & Sept & $82.8^{p}$ & $10.7^{p}$ & $25.3^{p}$ & $21.6^{p}$ & $13.9^{p}$ & $8.9^{p}$ & $2.4^{p}$ & $27.0^{p}$ & $45.5^{p}$ & $94.0^{p}$ & $72.3^{p}$ & $43.0^{P}$ & $28.2^{p}$ & $30.1^{p}$ & $36.2^{p}$ & $65.8^{p}$ & $20.5^{p}$ & $13.8^{p}$ \\
\hline
\end{tabular}

1 Unstandardised and therefore takes no account of the age structure of the population.

2 Births outside marriage can be registered by both the mother and father (joint) or by the mother alone (sole)

3 Usual address(es) of parents.

p provisional 
England and Wales (residents)

Numbers (thousands) and rates; and percentage terminated by abortion

\begin{tabular}{l|l|l|l|l|l|l|l|l|l|}
\cline { 2 - 7 } & \multicolumn{6}{|c|}{ Age of woman at conception } \\
\hline Year and quarter & All ages & Under 16 & Under 18 & Under 20 & $20-24$ & $25-29$ & $30-34$ & $35-39$ & 40 and over \\
\hline
\end{tabular}

\begin{tabular}{|c|c|c|}
\hline $\begin{array}{l}1991 \\
1996\end{array}$ & & $\begin{array}{l}853.7 \\
816.9\end{array}$ \\
\hline $\begin{array}{l}1999 \\
2000 \\
2001 \\
2002 \\
2003 \\
2004 \\
2005 \\
2006 \\
20071\end{array}$ & & $\begin{array}{l}774.0 \\
767.0 \\
763.7 \\
787.0 \\
806.8 \\
826.8 \\
841.8 \\
870.0 \\
894.1\end{array}$ \\
\hline 2004 & $\begin{array}{l}\text { March } \\
\text { June } \\
\text { Sept } \\
\text { Dec }\end{array}$ & $\begin{array}{l}207.9 \\
200.1 \\
203.6 \\
215.2\end{array}$ \\
\hline 2005 & $\begin{array}{l}\text { March } \\
\text { June } \\
\text { Sept } \\
\text { Dec }\end{array}$ & $\begin{array}{l}204.6 \\
204.7 \\
210.9 \\
221.7\end{array}$ \\
\hline 2006 & $\begin{array}{l}\text { March } \\
\text { June } \\
\text { Sept } \\
\text { Dec }\end{array}$ & $\begin{array}{l}214.0 \\
212.6 \\
215.1 \\
228.2\end{array}$ \\
\hline 2007 & $\begin{array}{l}\text { March'1,P } \\
\text { June } e^{1, P} \\
\text { Sept } t^{1, P} \\
\text { Dec }^{1, P}\end{array}$ & $\begin{array}{l}221.6 \\
221.1 \\
222.2 \\
229.2\end{array}$ \\
\hline
\end{tabular}

$\begin{array}{ll}7.5 & 40 . \\ 8.9 & 43 \\ 7.9 & 42 \\ 8.9 & 41 \\ 7.9 & 41 \\ 7.9 & 42 \\ 8.0 & 42 \\ 7.6 & 42 \\ 7.9 & 42 \\ 7.8 & 41 \\ 8.2 & 42 . \\ 2.0 & 10.9 \\ 1.9 & 10 \\ 1.8 & 10 \\ 1.9 & 10.8 \\ 1.9 & 10 \\ 2.0 & 10 \\ 2.0 & 10 \\ 2.0 & 11 .\end{array}$

(b) rates (conceptions per thousand women in age group)

\begin{tabular}{|c|c|c|c|}
\hline $\begin{array}{l}1991 \\
1996\end{array}$ & $\begin{array}{l}77.7 \\
76.2\end{array}$ & $\begin{array}{l}8.9 \\
9.5\end{array}$ & $\begin{array}{l}44.6 \\
46.3\end{array}$ \\
\hline $\begin{array}{l}1999 \\
2000 \\
2001 \\
2002 \\
2003 \\
2004 \\
2005 \\
2006 \\
20071,2, \mathrm{P}\end{array}$ & $\begin{array}{l}71.9 \\
70.9 \\
70.3 \\
72.2 \\
73.7 \\
75.2 \\
76.0 \\
78.3 \\
80.3\end{array}$ & $\begin{array}{l}8.3 \\
8.3 \\
8.0 \\
7.9 \\
7.9 \\
7.5 \\
7.8 \\
7.8 \\
8.3\end{array}$ & $\begin{array}{l}45.1 \\
43.9 \\
42.7 \\
42.9 \\
42.4 \\
41.8 \\
41.4 \\
40.9 \\
41.9\end{array}$ \\
\hline $\begin{array}{l}2004 \text { March } \\
\text { June } \\
\text { Sept } \\
\text { Dec }\end{array}$ & $\begin{array}{l}76.2 \\
73.2 \\
73.6 \\
77.7\end{array}$ & $\begin{array}{l}7.8 \\
7.7 \\
7.1 \\
7.4\end{array}$ & $\begin{array}{l}43.5 \\
42.2 \\
39.2 \\
42.4\end{array}$ \\
\hline $\begin{array}{l}2005 \text { March } \\
\text { June } \\
\text { Sept } \\
\text { Dec }\end{array}$ & $\begin{array}{l}75.1 \\
74.2 \\
75.5 \\
79.3\end{array}$ & $\begin{array}{l}7.6 \\
8.0 \\
7.8 \\
7.9\end{array}$ & $\begin{array}{l}41.5 \\
41.1 \\
40.5 \\
42.8\end{array}$ \\
\hline $\begin{array}{l}2006 \text { March } \\
\text { June } \\
\text { Sept } \\
\text { Dec }\end{array}$ & $\begin{array}{l}78.2 \\
76.7 \\
76.8 \\
81.4\end{array}$ & $\begin{array}{l}7.1 \\
8.2 \\
7.7 \\
8.1\end{array}$ & $\begin{array}{l}40.4 \\
41.4 \\
38.8 \\
42.8\end{array}$ \\
\hline 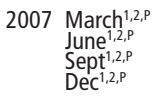 & $\begin{array}{l}80.8 \\
79.7 \\
79.2 \\
81.6\end{array}$ & $\begin{array}{l}8.1 \\
8.7 \\
8.2 \\
8.3\end{array}$ & $\begin{array}{l}42.9 \\
42.8 \\
40.1 \\
42.1\end{array}$ \\
\hline
\end{tabular}

$\begin{array}{ll}44.6 & 64.1 \\ 46.3 & 63.2 \\ 45.1 & 63.1 \\ 43.9 & 62.5 \\ 42.7 & 60.8 \\ 42.9 & 60.6 \\ 42.4 & 60.0 \\ 41.8 & 60.3 \\ 41.4 & 60.1 \\ 40.9 & 60.2 \\ 41.9 & 61.5 \\ 43.5 & 63.2 \\ 42.2 & 60 . \\ 39.2 & 56.8 \\ 42.4 & 61.5 \\ 41.5 & 60.0 \\ 41.1 & 59.1 \\ 40.5 & 59.0 \\ 42.8 & 62.4 \\ 40.4 & 60.3 \\ 41.4 & 60.3 \\ 38.8 & 57.2 \\ 42.8 & 63.0 \\ 42.9 & 62.9 \\ 42.8 & 62.6 \\ 40.1 & 59.0 \\ 42.1 & 62 . \\ & \end{array}$

\begin{tabular}{rr}
101.6 & 233. \\
94.9 & 179.8 \\
98.8 & 157. \\
97.7 & 159. \\
96.0 & 161. \\
97.1 & 167. \\
98.6 & 175. \\
101.3 & 181. \\
102.3 & 185. \\
103.1 & 191. \\
106.1 & 198. \\
26.2 & 45.9 \\
25.0 & 43. \\
24.0 & 44. \\
26.1 & 47. \\
25.1 & 45. \\
25.1 & 45. \\
25.3 & 45. \\
26.8 & 49.3 \\
25.4 & 47.5 \\
25.7 & 46. \\
24.7 & 46. \\
27.3 & 50. \\
26.7 & 49.4 \\
26.9 & 49. \\
25.6 & 48.5 \\
26.9 & 51. \\
\hline
\end{tabular}

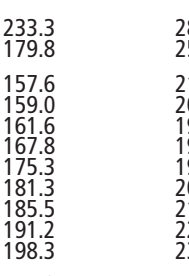

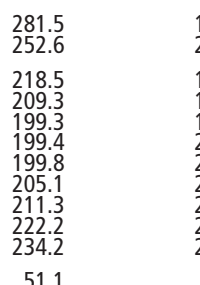

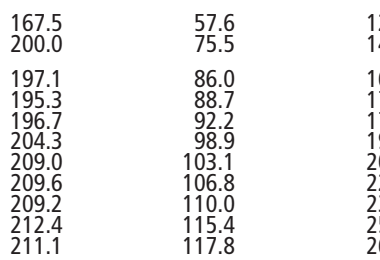

$12: 1$<smiles>[131IH]</smiles>

44.7

52.6
50.4
54.0
54.0

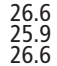

54.0

$\begin{array}{ll}45.4 & 5.8 \\ 45.2 & 55.0 \\ 45.6 & 53.0 \\ 45.6 & 5.2\end{array}$

$1.8 \quad 10.2$

51.0
53.3
56.2

51.0
50.7
53.3

$\begin{array}{ll}47.5 & 5.2 \\ 46.9 & 53.8 \\ 46.3 & 55.3 \\ 5.6 & 5.9 \\ 5.9 & -9.9\end{array}$

49.4
49.3 57.0

48.5
41.0

$64.1 \quad 120.2$

$120.2-135$

$135.1-90.1$

27.6

26.6
27.5
27.5

54.3

16.0

17.8
19.6

$\begin{array}{ll}3.1 & 103.9 \\ 5 & 103\end{array}$

$\begin{array}{ll}103.9 & 118.0 \\ 103.2 & 115.7\end{array}$

118.0
115.7
114.2
119.0

107.2

107.2
109.0
108.7

109.5
111.4

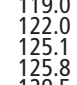

125.1
125.8
129.5
133.4

111.5
105.9
105.0

129.5
133.4
125.4

125.4
121.1
122.6
129.9

105.9

108.9
106.7
105.7

$\begin{array}{ll}60.0 & 108.9 \\ 59.1 & 106.7 \\ 59.0 & 105.7\end{array}$

123.8

$\begin{array}{ll}60.3 & 111.2 \\ 60.3 & 108.1\end{array}$

$\begin{array}{ll}57.2 & 104.8 \\ 63.0 & 114.1\end{array}$

129.2

$\begin{array}{ll}114.1 & 127.4 \\ 113.4 & 134.9\end{array}$

$\begin{array}{ll}62.9 & 113.4 \\ 62.6 & 111.3 \\ 59.0 & 107.8 \\ 62.1 & 112.6\end{array}$

132.8
131.6
132.2
136.2

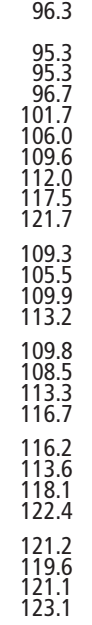

8.4

(c) percentage terminated by abortion

1991
1996
1999
2000
2001
2002
2003
2004
2005
2006
$20077^{1, p}$

$\begin{array}{ll}19.4 & 5 \\ 20.8 & 49.2 \\ 22.6 & 55.6 \\ 22.7 & 55.0 \\ 23.2 & 55.8 \\ 22.5 & 55.6 \\ 22.5 & 5 \\ 22.4 & 5.2 \\ 22.2 & 55.3 \\ 22.0 & 6\end{array}$

$\begin{array}{ll}51.1 & 39 . \\ 49.2 & 40.0 \\ 52.6 & 43 \\ 54.0 & 44 \\ 55.8 & 45 \\ 55.6 & 45 \\ 57.4 & 45 \\ 57.2 & 45 . \\ 57.1 & 46 \\ 59.8 & 48 \\ 61.4 & 50 .\end{array}$

39.9
40.0

$\begin{array}{ll}34.5 \\ 36.2\end{array} 22$.

$\begin{array}{ll}22.2 & 134 \\ 25.7 & 135 \\ 28.5 & 175 \\ 29.2 & 172 \\ 29.7 & 184 \\ 28.8 & 179 \\ 29.0 & 179 \\ 28.9 & 182 \\ 28.7 & 180 \\ 28.3 & 175 \\ 2.3 & \end{array}$

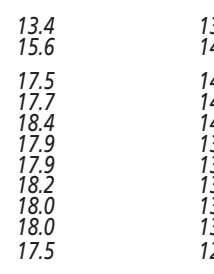

13.7
14.1
14.7
14.5

34.4
40.7

9.1

42.9
43.2

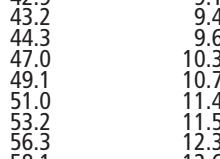

10.3
10.7
$11: 4$
11.5
12.3
12.5

51.7
49.7
50.6

11.5

11.4

11.4
$11: 4$
11.7

51.8
52.0
52.8
55.9

12.2
12.5
12.1

12.6
12.5
12.6
12.8

\begin{tabular}{cccc}
2004 March & 22.7 & 58.2 & 45.7 \\
June & 23.0 & 57.2 & 46.3 \\
Sept & 21.9 & 56.8 & 45.8 \\
Dec & 22.0 & 56.3 & 44.5 \\
2005 March & 22.5 & 57.5 & 47.3 \\
June & 22.7 & 57.0 & 45.8 \\
Sept & 21.4 & 56.2 & 45.3 \\
Dec & 22.2 & 57.5 & 46.9 \\
2006 March & 22.5 & 59.0 & 47.7 \\
June & 23.1 & 59.5 & 49.0 \\
Sept & 21.5 & 60.4 & 48.0 \\
Dec & 22.0 & 60.2 & 49.1 \\
2007 March Mar. $^{1,8}$ & 22.7 & 62.5 & 50.7 \\
June & 22.5 & 61.9 & 50.3 \\
Sept $^{1, p}$ & 21.1 & 57.9 & 48.6 \\
\hline
\end{tabular}

Dec $^{1, P}$

21.1
21.9

57.9
63.3

48.6
50.4

29.4
29.2
28.4
28.6

18.5
18.6
17.9
17.8

55.3
56.0
58.2

58.4
57.2
58.3
58.7

Note: Conception figures are estimates derived from birth registrations and abortion notifications.

Rates for women of all ages, under 16, under 18, under 20 and 40 and over are based on the population of women aged 15-44, 13-15, 15-17, 15-19 and 40-44 respectively.

For a quarterly analysis of conceptions to women under 18 for local authority areas see the ONS website, www.ons.gov.uk

1 Figures for conceptions in 2007 exclude maternities relating to births in 2008 where the mother's date of birth was not stated on the registration and could not be supplied from another source. See Explanatory notes in the 2007 Conceptions report in this volume.

2 Rates for both quarterly and annual 2007 conceptions are calculated using the 2007 mid-year estimates.

p provisional 


\begin{tabular}{|c|c|c|c|c|c|c|c|c|c|c|c|c|c|c|c|}
\hline \multirow{2}{*}{\multicolumn{2}{|c|}{$\begin{array}{l}\text { Table } 4.2 \\
\text { England and Wales }\end{array}$}} & \multicolumn{14}{|c|}{ Abortions: residents and non-residents; age and gestation (residents only) } \\
\hline & & \multicolumn{14}{|c|}{ Numbers (thousands) and rates; and percentages for gestation weeks } \\
\hline & & & & & \multicolumn{11}{|c|}{ All women (residents) } \\
\hline & & \multicolumn{3}{|c|}{ All ages } & \multicolumn{7}{|c|}{ Age group } & \multicolumn{4}{|c|}{ Gestation weeks (percentages) } \\
\hline \multicolumn{2}{|c|}{ Year and quarter } & $\begin{array}{c}\text { All' } \\
\text { women }\end{array}$ & Residents' & $\begin{array}{l}\text { Non-1 } \\
\text { residents }\end{array}$ & $\begin{array}{c}\text { Under } \\
16\end{array}$ & $16-19$ & $20-24$ & $25-29$ & 30-34 & $35-44$ & $\begin{array}{c}45 \text { and } \\
\text { over }\end{array}$ & $\begin{array}{c}\text { Under } \\
9\end{array}$ & $9-12$ & 13-19 & $\begin{array}{c}20 \text { and } \\
\text { over }\end{array}$ \\
\hline & & \multicolumn{3}{|c|}{ Numbers (thousands) } & & & & & & & & \multicolumn{2}{|c|}{ Percentages } & & \\
\hline $\begin{array}{l}19912 \\
1976 \\
1981 \\
1986 \\
1991 \\
1996\end{array}$ & & $\begin{array}{l}122.8 \\
129.7 \\
162.5 \\
172.3 \\
179.5 \\
177.5\end{array}$ & $\begin{array}{l}94.6 \\
101.9 \\
128.6 \\
147.6 \\
167.4 \\
167.9\end{array}$ & $\begin{array}{l}32.2 \\
27.8 \\
33.9 \\
24.7 \\
12.1 \\
9.6\end{array}$ & $\begin{array}{l}2.3 \\
3.4 \\
3.5 \\
3.5 \\
3.9 \\
3.2\end{array}$ & $\begin{array}{l}18.2 \\
24.0 \\
31.4 \\
33.8 \\
31.1 \\
28.8\end{array}$ & $\begin{array}{l}24.5 \\
23.6 \\
34.3 \\
45.3 \\
52.7 \\
46.4\end{array}$ & $\begin{array}{l}17.3 \\
19.3 \\
21.9 \\
28.7 \\
38.6 \\
39.3\end{array}$ & $\begin{array}{l}14.2 \\
14.6 \\
18.7 \\
18.0 \\
23.4 \\
28.2\end{array}$ & $\begin{array}{l}15.9 \\
14.7 \\
17.6 \\
17.5 \\
17.9 \\
21.1\end{array}$ & $\begin{array}{l}0.5 \\
0.5 \\
0.6 \\
0.4 \\
0.4 \\
0.4\end{array}$ & $\begin{array}{l}16.6 \\
24.8 \\
31.0 \\
33.4 \\
35.2 \\
40.0\end{array}$ & $\begin{array}{l}57.9 \\
55.8 \\
53.4 \\
53.8 \\
52.9 \\
48.7\end{array}$ & $\begin{array}{l}21.8 \\
15.0 \\
13.5 \\
11.5 \\
10.6 \\
10.1\end{array}$ & $\begin{array}{l}1.0 \\
1.1 \\
1.3 \\
1.4 \\
1.2\end{array}$ \\
\hline $\begin{array}{l}1997 \\
1998 \\
1999 \\
2000 \\
2001\end{array}$ & & $\begin{array}{l}179.7 \\
187.4 \\
188.2 \\
185.4 \\
186.3\end{array}$ & $\begin{array}{l}170.1 \\
177.9 \\
173.7 \\
1755.5 \\
176.4\end{array}$ & $\begin{array}{l}9.6 \\
9.5 \\
9.5 \\
9.8 \\
9.9\end{array}$ & $\begin{array}{l}3.4 \\
3.8 \\
3.6 \\
3.6 \\
3.7\end{array}$ & $\begin{array}{l}29.9 \\
33.2 \\
32.8 \\
33.2 \\
33.4\end{array}$ & $\begin{array}{l}45.0 \\
45.8 \\
45.0 \\
47.1 \\
48.3\end{array}$ & $\begin{array}{l}40.2 \\
40.4 \\
38.5 \\
37.9 \\
36.5\end{array}$ & $\begin{array}{l}28.9 \\
330.4 \\
29.1 \\
28.7 \\
28.8\end{array}$ & $\begin{array}{l}22.3 \\
23.8 \\
24.1 \\
24.4 \\
25.2\end{array}$ & $\begin{array}{l}0.5 \\
0.5 \\
0.5 \\
0.5 \\
0.5\end{array}$ & $\begin{array}{l}41.2 \\
41.4 \\
42.5 \\
43.3 \\
42.8\end{array}$ & $\begin{array}{l}47.9 \\
47.6 \\
46.5 \\
45.0 \\
45.0\end{array}$ & $\begin{array}{l}9.6 \\
9.7 \\
9.5 \\
10.3 \\
10.6\end{array}$ & $\begin{array}{l}1.2 \\
1.3 \\
1.4 \\
1.5 \\
1.6\end{array}$ \\
\hline $\begin{array}{l}2002 \\
2003 \\
2004 \\
2005\end{array}$ & & $\begin{array}{l}185.4 \\
190.7 \\
194.5 \\
194.4\end{array}$ & $\begin{array}{l}175.9 \\
181.6 \\
185.7 \\
186.4\end{array}$ & $\begin{array}{l}9.5 \\
99.1 \\
8.8\end{array}$ & $\begin{array}{l}3.7 \\
4.0 \\
3.8 \\
3.8\end{array}$ & $\begin{array}{l}33.0 \\
34.2 \\
35.5 \\
35.3\end{array}$ & $\begin{array}{l}48.4 \\
51.1 \\
52.8 \\
53.3\end{array}$ & $\begin{array}{l}35.8 \\
36.0 \\
37.8 \\
38.3\end{array}$ & $\begin{array}{l}28.5 \\
28.7 \\
28.1 \\
27.8\end{array}$ & $\begin{array}{l}26.0 \\
26.9 \\
27.3 \\
27.2\end{array}$ & $\begin{array}{l}0.5 \\
0.5 \\
0.5 \\
0.6\end{array}$ & $\begin{array}{l}42.2 \\
43.6 \\
46.2 \\
53.6\end{array}$ & $\begin{array}{l}45.2 \\
43.7 \\
41.5 \\
35.7\end{array}$ & $\begin{array}{l}11.0 \\
11.1 \\
10.8 \\
9.3\end{array}$ & $\begin{array}{l}1.6 \\
1.6 \\
1.6\end{array}$ \\
\hline 2006 & & $\begin{array}{l}201.2 \\
205.6\end{array}$ & $\begin{array}{l}193.7 \\
198.5\end{array}$ & 7.4 & $\begin{array}{l}4.0 \\
4.4\end{array}$ & $\begin{array}{l}37.3 \\
39.6\end{array}$ & $\begin{array}{l}55.3 \\
57.0\end{array}$ & $\begin{array}{l}40.4 \\
41.7\end{array}$ & 28.2 & $\begin{array}{l}27.9 \\
27.9\end{array}$ & $\begin{array}{l}0.7 \\
0.7\end{array}$ & $\begin{array}{l}54.9 \\
57.9\end{array}$ & $\begin{array}{l}34.3 \\
31.9\end{array}$ & $\begin{array}{l}9.2 \\
8.8\end{array}$ & 1.5 \\
\hline 2004 & $\begin{array}{l}\text { March } \\
\text { June } \\
\text { Sept } \\
\text { Dec }\end{array}$ & $\begin{array}{l}51.1 \\
48.9 \\
48.9 \\
46.1\end{array}$ & $\begin{array}{l}48.7 \\
46.6 \\
46.3 \\
44.2\end{array}$ & $\begin{array}{l}2.4 \\
2.3 \\
2.1 \\
1.9\end{array}$ & $\begin{array}{l}1.0 \\
1.0 \\
1.0 \\
1.0\end{array}$ & $\begin{array}{l}9.4 \\
8.9 \\
8.9 \\
8.4\end{array}$ & $\begin{array}{l}13.9 \\
13.3 \\
13.0 \\
12.6\end{array}$ & $\begin{array}{l}9.8 \\
9.5 \\
9.4 \\
9.1\end{array}$ & $\begin{array}{l}7.5 \\
6.9 \\
7.0 \\
6.6\end{array}$ & $\begin{array}{l}7.0 \\
6.99 \\
6.99 \\
6.5\end{array}$ & $\begin{array}{l}0.1 \\
0.1 \\
0.1 \\
0.1\end{array}$ & $\begin{array}{l}41.7 \\
43.6 \\
47.8 \\
52.0\end{array}$ & $\begin{array}{l}44.5 \\
43.3 \\
40.5 \\
37.2\end{array}$ & $\begin{array}{l}12.1 \\
11.2 \\
10.3 \\
9.5\end{array}$ & $\begin{array}{l}1.7 \\
1.8 \\
1.4\end{array}$ \\
\hline 2005 & $\begin{array}{l}\text { March } \\
\text { June } \\
\text { Sept } \\
\text { Dec }\end{array}$ & $\begin{array}{l}50.1 \\
50.1 \\
47.0 \\
47.2\end{array}$ & $\begin{array}{l}47.9 \\
48.0 \\
45.1 \\
45.3\end{array}$ & $\begin{array}{l}2.1 \\
2.1 \\
1.9 \\
1.8\end{array}$ & $\begin{array}{l}0.9 \\
1.0 \\
1.0 \\
0.9\end{array}$ & $\begin{array}{l}9.1 \\
9.2 \\
8.5 \\
8.6\end{array}$ & $\begin{array}{l}13.9 \\
13.9 \\
12.7 \\
12.9\end{array}$ & $\begin{array}{l}9.7 \\
9.9 \\
9.3 \\
9.5\end{array}$ & $\begin{array}{l}7.2 \\
7.1 \\
6.9 \\
6.7\end{array}$ & $\begin{array}{l}7.0 \\
6.99 \\
6.7\end{array}$ & $\begin{array}{l}0.1 \\
0.1 \\
0.1 \\
0.1\end{array}$ & $\begin{array}{l}47.2 \\
53.8 \\
56.5 \\
57.2\end{array}$ & $\begin{array}{l}40.4 \\
35.6 \\
33.6 \\
32.9\end{array}$ & $\begin{array}{l}11.0 \\
9.2 \\
8.5 \\
8.3\end{array}$ & $\begin{array}{l}1.4 \\
1.4 \\
1.5\end{array}$ \\
\hline 2006 & $\begin{array}{l}\text { March } \\
\text { June } \\
\text { Sept } \\
\text { Dec }\end{array}$ & $\begin{array}{l}52.4 \\
51.3 \\
49.8 \\
47.7\end{array}$ & $\begin{array}{l}50.4 \\
49.3 \\
47.9 \\
46.0\end{array}$ & $\begin{array}{l}2.0 \\
2: 0 \\
1.8 \\
1.6\end{array}$ & $\begin{array}{l}1.0 \\
1.0 \\
1.0 \\
1.0\end{array}$ & $\begin{array}{l}9.8 \\
9.4 \\
9.2 \\
8.8\end{array}$ & $\begin{array}{l}14.6 \\
14.2 \\
13.6 \\
13.0\end{array}$ & $\begin{array}{l}10.4 \\
10.3 \\
10.0 \\
9.6\end{array}$ & $\begin{array}{l}7.2 \\
7.2 \\
7.0 \\
6.7\end{array}$ & $\begin{array}{l}7.3 \\
7.1 \\
6.9 \\
6.7\end{array}$ & $\begin{array}{l}0.2 \\
0.2 \\
0.2 \\
0.1\end{array}$ & $\begin{array}{l}50.6 \\
53.6 \\
56.5 \\
59.5\end{array}$ & $\begin{array}{l}37.3 \\
35.3 \\
33.0 \\
31.3\end{array}$ & $\begin{array}{l}10.5 \\
9.4 \\
9.0 \\
7.9\end{array}$ & $\begin{array}{l}1.6 \\
1.7 \\
1.5\end{array}$ \\
\hline 2007 & $\begin{array}{l}\text { March } \\
\text { June } \\
\text { Sept } \\
\text { Dec }\end{array}$ & $\begin{array}{l}55.3 \\
51.2 \\
49.9 \\
49.2\end{array}$ & $\begin{array}{l}53.3 \\
49.4 \\
48.2 \\
47.6\end{array}$ & $\begin{array}{l}1.9 \\
1.8 \\
1.7\end{array}$ & $\begin{array}{l}1.2 \\
1.1 \\
1.0 \\
1.1\end{array}$ & $\begin{array}{c}10.8 \\
9.9 \\
9.5 \\
9.4\end{array}$ & $\begin{array}{l}15.4 \\
15.3 \\
13.5 \\
13.7\end{array}$ & $\begin{array}{l}11.1 \\
10.3 \\
10.2 \\
10.0\end{array}$ & $\begin{array}{l}7.3 \\
6.7 \\
6.6 \\
6.6\end{array}$ & $\begin{array}{l}7.4 \\
6.8 \\
7.0 \\
6.7\end{array}$ & $\begin{array}{l}0.2 \\
0.2 \\
0.2 \\
0.2\end{array}$ & $\begin{array}{l}54.1 \\
56.6 \\
58.6 \\
62.7\end{array}$ & $\begin{array}{l}35.0 \\
32.6 \\
31.2 \\
28.3\end{array}$ & $\begin{array}{l}9.4 \\
9.2 \\
8.8 \\
7.6\end{array}$ & $\begin{array}{l}1.6 \\
1.5 \\
1.4\end{array}$ \\
\hline 2008 & $\begin{array}{l}\text { Marchp } \\
\text { Junep } \\
\text { Seppte }\end{array}$ & $\begin{array}{l}53.3 \\
52.5 \\
48.4\end{array}$ & $\begin{array}{l}51.5 \\
50.8 \\
46.7\end{array}$ & $\begin{array}{l}1.8 \\
1.8 \\
1.7\end{array}$ & $\begin{array}{l}1: 1 \\
1: 1 \\
1.0\end{array}$ & $\begin{array}{l}10.5 \\
10.1 \\
9.1\end{array}$ & $\begin{array}{l}14.7 \\
4.6 \\
13.3\end{array}$ & $\begin{array}{l}10.8 \\
10.9 \\
10.2\end{array}$ & $\begin{array}{l}7.0 \\
6.9 \\
6.5\end{array}$ & $\begin{array}{l}7.1 \\
7.0 \\
6.4\end{array}$ & $\begin{array}{l}0.2 \\
0.2 \\
0.2\end{array}$ & $\begin{array}{l}58.4 \\
60.8 \\
62.9\end{array}$ & $\begin{array}{l}31.0 \\
29.4 \\
27.6\end{array}$ & $\begin{array}{l}9.0 \\
8.3 \\
8.0\end{array}$ & $\begin{array}{l}1.6 \\
1.4 \\
1.5\end{array}$ \\
\hline \multicolumn{16}{|c|}{$\begin{array}{l}\text { Rates (per thousand women residents) } \\
\text { ASR R } \\
\text { (women 15-44) (women 15te-44) }\end{array}$} \\
\hline $\begin{array}{l}1971 \\
1976 \\
1981 \\
1986 \\
1991 \\
1996\end{array}$ & & $\begin{array}{r}9.9 \\
10.2 \\
11.9 \\
13.0 \\
15.0 \\
16.0\end{array}$ & $\begin{array}{l}10.1 \\
10.5 \\
12.4 \\
13.5 \\
15.2 \\
15.7\end{array}$ & & $\begin{array}{l}2.3 \\
2.9 \\
3.0 \\
3.7 \\
3.8 \\
3.9 \\
3.9\end{array}$ & $\begin{array}{l}13.9 \\
16.9 \\
19.4 \\
22.0 \\
24.0 \\
24.2\end{array}$ & $\begin{array}{l}13.1 \\
14.2 \\
18.6 \\
21.9 \\
27.1 \\
28.4\end{array}$ & $\begin{array}{l}10.7 \\
10.4 \\
13.1 \\
15.5 \\
18.5 \\
19.9\end{array}$ & $\begin{array}{l}10.0 \\
9.2 \\
10.1 \\
10.8 \\
12.6 \\
13.6\end{array}$ & $\begin{array}{l}5.6 \\
5.3 \\
5.9 \\
5.1 \\
5.1 \\
6.0\end{array}$ & $\begin{array}{l}0.3 \\
0.3 \\
0.4 \\
0.3 \\
0.3 \\
0.2\end{array}$ & & & & \\
\hline $\begin{array}{l}1997 \\
1998 \\
1999 \\
2000 \\
2001\end{array}$ & & $\begin{array}{l}16.3 \\
17.1 \\
16.8 \\
17.0 \\
17.0\end{array}$ & $\begin{array}{l}15.9 \\
16.9 \\
16.2 \\
16.2 \\
16.3 \\
16.2\end{array}$ & & $\begin{array}{l}3.7 \\
4.0 \\
3.8 \\
3.9 \\
3.7\end{array}$ & $\begin{array}{l}24.4 \\
26.8 \\
26.3 \\
26.9 \\
26.6\end{array}$ & $\begin{array}{l}28.8 \\
30.2 \\
29.7 \\
30.7 \\
30.6\end{array}$ & $\begin{array}{l}20.7 \\
21.2 \\
20.8 \\
20.9 \\
20.9\end{array}$ & $\begin{array}{l}13.8 \\
14.6 \\
14.1 \\
14.1 \\
14.2\end{array}$ & $\begin{array}{l}6.2 \\
6.5 \\
6.4 \\
6.3 \\
6.4\end{array}$ & $\begin{array}{l}0.3 \\
0.3 \\
0.3 \\
0.3 \\
0.3\end{array}$ & & & & \\
\hline $\begin{array}{l}2002 \\
2003 \\
2004 \\
2005\end{array}$ & & $\begin{array}{l}17.0 \\
17.5 \\
17.8 \\
17.8\end{array}$ & $\begin{array}{l}16.1 \\
16.6 \\
16.9 \\
17.0\end{array}$ & & $\begin{array}{l}3.7 \\
3.9 \\
3.7 \\
3.7\end{array}$ & $\begin{array}{l}25.8 \\
26.1 \\
26.5 \\
26.3\end{array}$ & $\begin{array}{l}30.1 \\
3.2 \\
31.9 \\
32.0\end{array}$ & $\begin{array}{l}21.4 \\
22.1 \\
22.3 \\
23.6\end{array}$ & $\begin{array}{l}14.2 \\
14.6 \\
14.7 \\
14.5\end{array}$ & $\begin{array}{l}6.5 \\
6.6 \\
6.7 \\
6.6\end{array}$ & $\begin{array}{l}0.3 \\
0.3 \\
0.3 \\
0.3\end{array}$ & & & & \\
\hline 2006 & & $\begin{array}{l}18.3 \\
18.6\end{array}$ & $\begin{array}{l}17.5 \\
17.9\end{array}$ & & $\begin{array}{l}3.9 \\
4.4\end{array}$ & $\begin{array}{l}27.3 \\
28.9\end{array}$ & $\begin{array}{l}32.5 \\
32.6\end{array}$ & 24.3 & 15.1 & 6.8 & 0.4 & & & & \\
\hline 2004 & $\begin{array}{l}\text { March } \\
\text { June } \\
\text { Sept } \\
\text { Dec }\end{array}$ & $\begin{array}{l}18.7 \\
17.9 \\
17.8 \\
17.0\end{array}$ & $\begin{array}{l}17.8 \\
17.0 \\
16.9 \\
16.2\end{array}$ & & $\begin{array}{l}3.9 \\
3.9 \\
3.7 \\
3.5\end{array}$ & $\begin{array}{l}28.3 \\
26.7 \\
26.6 \\
25.0\end{array}$ & $\begin{array}{l}33.8 \\
32.3 \\
31.5 \\
30.4\end{array}$ & $\begin{array}{l}24.1 \\
22.3 \\
22.0 \\
22.3\end{array}$ & $\begin{array}{l}15.4 \\
14.4 \\
14.8 \\
14.2\end{array}$ & $\begin{array}{l}6.9 \\
6.7 \\
6.8 \\
6.3\end{array}$ & $\begin{array}{l}0.3 \\
0.3 \\
0.3 \\
0.3\end{array}$ & & & & \\
\hline 2005 & $\begin{array}{l}\text { March } \\
\text { June } \\
\text { Sept } \\
\text { Dec }\end{array}$ & $\begin{array}{l}18.4 \\
18.4 \\
17.3 \\
17.4\end{array}$ & $\begin{array}{l}17.5 \\
17.5 \\
16.4 \\
16.5\end{array}$ & & $\begin{array}{l}3.7 \\
3.8 \\
3.8 \\
3.6\end{array}$ & $\begin{array}{l}27.0 \\
27.2 \\
25.2 \\
25.4\end{array}$ & $\begin{array}{l}33.5 \\
33.3 \\
33.5 \\
30.9\end{array}$ & $\begin{array}{l}23.8 \\
24.1 \\
22.6 \\
23.0\end{array}$ & $\begin{array}{l}15.2 \\
15.3 \\
14.8 \\
14.4\end{array}$ & $\begin{array}{l}6.8 \\
6.7 \\
6.5 \\
6.5\end{array}$ & $\begin{array}{l}0.3 \\
0.3 \\
0.3 \\
0.3\end{array}$ & & & & \\
\hline 2006 & $\begin{array}{l}\text { March } \\
\text { June } \\
\text { Sept } \\
\text { Dec }\end{array}$ & $\begin{array}{l}19.3 \\
18.9 \\
18.3 \\
17.5\end{array}$ & $\begin{array}{l}\begin{array}{l}18.4 \\
18.0 \\
17.5 \\
16.8\end{array} \\
16.8\end{array}$ & & $\begin{array}{l}3.9 \\
3.9 \\
4.0 \\
4.0\end{array}$ & $\begin{array}{l}29.0 \\
27.8 \\
27.0 \\
25.9\end{array}$ & $\begin{array}{l}34.8 \\
33.8 \\
32.2 \\
30.8\end{array}$ & $\begin{array}{l}25.0 \\
24.7 \\
22.9 \\
22.8\end{array}$ & $\begin{array}{l}15.9 \\
16.0 \\
15.9 \\
15.3\end{array}$ & $\begin{array}{l}7.0 \\
6.8 \\
6.7 \\
6.5\end{array}$ & $\begin{array}{l}0.3 \\
0.4 \\
0.4 \\
0.3\end{array}$ & & & & \\
\hline 2007 & $\begin{array}{l}\text { March } \\
\text { June } \\
\text { Dect } \\
\text { Dec }\end{array}$ & $\begin{array}{l}20.0 \\
18.5 \\
18.0 \\
17.8\end{array}$ & $\begin{array}{l}19.2 \\
17.8 \\
17.4 \\
17.2\end{array}$ & & $\begin{array}{l}4.7 \\
4.4 \\
4.2 \\
4.4\end{array}$ & $\begin{array}{l}31.4 \\
28.8 \\
27.7 \\
27.2\end{array}$ & $\begin{array}{l}35.3 \\
32.7 \\
30.8 \\
31.1\end{array}$ & $\begin{array}{l}25.6 \\
22.7 \\
22.3 \\
22.7\end{array}$ & $\begin{array}{l}16.7 \\
15.5 \\
15.4 \\
15.3\end{array}$ & $\begin{array}{l}7.2 \\
6.6 \\
6.8 \\
6.5\end{array}$ & $\begin{array}{l}0.4 \\
0.4 \\
0.4 \\
0.4\end{array}$ & & & & \\
\hline 2008 & $\begin{array}{l}\text { Marcch } \\
\text { Junep } \\
\text { Sept }\end{array}$ & $\begin{array}{l}19.3 \\
19.0 \\
17.4\end{array}$ & $\begin{array}{l}18.6 \\
18.3 \\
16.9\end{array}$ & & $\begin{array}{l}4.6 \\
4.4 \\
4.0\end{array}$ & $\begin{array}{l}30.4 \\
29.5 \\
26.6\end{array}$ & $\begin{array}{l}33.2 \\
33.8 \\
29.7\end{array}$ & $\begin{array}{l}24.3 \\
24.4 \\
22.7\end{array}$ & $\begin{array}{l}16.5 \\
16.3 \\
15.3\end{array}$ & $\begin{array}{l}7.0 \\
6.8 \\
6.3\end{array}$ & $\begin{array}{l}0.4 \\
0.3 \\
0.4\end{array}$ & & & & \\
\hline
\end{tabular}

Notes: Rates for under 16 and 45 and over are based on female populations aged 13-15 and 45-49 respectively.

1 Includes cases with not stated age and/or gestation week.

p provisional 
Constituent countries of the United Kingdom

\begin{tabular}{|c|c|c|c|c|c|c|c|c|c|c|c|c|c|c|c|c|c|}
\hline \multirow{3}{*}{ Year } & \multicolumn{8}{|c|}{ Males } & \multirow{3}{*}{ Year } & \multicolumn{8}{|c|}{ Females } \\
\hline & \multirow{2}{*}{$\underset{\text { birth }}{\mathrm{At}}$} & \multicolumn{7}{|c|}{ At age } & & \multirow{2}{*}{$\begin{array}{c}\text { At } \\
\text { birth }\end{array}$} & \multicolumn{7}{|c|}{ At age } \\
\hline & & 5 & 20 & 30 & 50 & 60 & 70 & 80 & & & 5 & 20 & 30 & 50 & 60 & 70 & 80 \\
\hline
\end{tabular}

\begin{tabular}{|c|c|c|c|c|c|c|c|c|c|c|c|c|c|c|c|c|c|}
\hline $\begin{array}{l}\text { United Kingdom } \\
1981 \\
1986 \\
1991 \\
1996\end{array}$ & $\begin{array}{l}70.8 \\
71.9 \\
73.2 \\
74.2\end{array}$ & $\begin{array}{l}66.9 \\
67.8 \\
68.9 \\
69.8\end{array}$ & $\begin{array}{l}52.3 \\
53.2 \\
54.2 \\
55.1\end{array}$ & $\begin{array}{l}42.7 \\
43.6 \\
44.7 \\
45.6\end{array}$ & $\begin{array}{l}24.1 \\
24.9 \\
26.0 \\
26.9\end{array}$ & $\begin{array}{l}16.3 \\
16.8 \\
17.7 \\
18.5\end{array}$ & $\begin{array}{l}10.1 \\
10.5 \\
11.1 \\
11.6\end{array}$ & $\begin{array}{l}5.8 \\
6.0 \\
6.4 \\
6.6\end{array}$ & $\begin{array}{l}1981 \\
1986 \\
1991 \\
1996\end{array}$ & $\begin{array}{l}76.8 \\
77.7 \\
78.7 \\
79.4\end{array}$ & $\begin{array}{l}72.7 \\
73.4 \\
74.3 \\
74.9\end{array}$ & $\begin{array}{l}57.9 \\
58.6 \\
59.5 \\
60.1\end{array}$ & $\begin{array}{l}48.2 \\
48.8 \\
49.7 \\
50.3\end{array}$ & $\begin{array}{l}29.2 \\
29.8 \\
30.6 \\
31.2\end{array}$ & $\begin{array}{l}20.8 \\
21.2 \\
21.9 \\
22.3\end{array}$ & $\begin{array}{l}13.3 \\
13.8 \\
14.3 \\
14.5\end{array}$ & $\begin{array}{l}7.5 \\
7.8 \\
8.2 \\
8.3\end{array}$ \\
\hline $\begin{array}{l}2000 \\
2001 \\
2002 \\
2003 \\
2004 \\
2005 \\
2006\end{array}$ & $\begin{array}{l}75.3 \\
75.6 \\
75.9 \\
76.2 \\
76.5 \\
76.9 \\
77.2\end{array}$ & $\begin{array}{l}70.9 \\
71.2 \\
71.4 \\
71.7 \\
72.0 \\
72.4 \\
72.7\end{array}$ & $\begin{array}{l}56.1 \\
56.4 \\
56.6 \\
56.9 \\
57.3 \\
57.6 \\
57.9\end{array}$ & $\begin{array}{l}46.6 \\
46.9 \\
47.1 \\
47.4 \\
47.7 \\
48.0 \\
48.3\end{array}$ & $\begin{array}{l}28.0 \\
28.2 \\
28.5 \\
28.7 \\
29.0 \\
29.4 \\
29.6\end{array}$ & $\begin{array}{l}19.5 \\
19.7 \\
19.9 \\
20.2 \\
20.5 \\
20.8 \\
21.1\end{array}$ & $\begin{array}{l}12.3 \\
12.5 \\
12.6 \\
12.8 \\
13.1 \\
13.4 \\
13.6\end{array}$ & $\begin{array}{l}7.0 \\
7.1 \\
7.1 \\
7.3 \\
7.4 \\
7.6 \\
7.7\end{array}$ & $\begin{array}{l}2000 \\
2001 \\
2002 \\
2003 \\
2004 \\
2005 \\
2006\end{array}$ & $\begin{array}{l}80.1 \\
80.4 \\
80.5 \\
80.7 \\
80.9 \\
81.3 \\
81.5\end{array}$ & $\begin{array}{l}75.6 \\
75.8 \\
75.9 \\
76.1 \\
76.4 \\
76.7 \\
76.9\end{array}$ & $\begin{array}{l}60.8 \\
61.0 \\
61.1 \\
61.3 \\
61.5 \\
61.9 \\
62.0\end{array}$ & $\begin{array}{l}51.0 \\
51.2 \\
51.3 \\
51.5 \\
51.7 \\
52.0 \\
52.2\end{array}$ & $\begin{array}{l}31.9 \\
32.1 \\
32.2 \\
32.4 \\
32.6 \\
32.9 \\
33.1\end{array}$ & $\begin{array}{l}23.0 \\
23.2 \\
23.3 \\
23.4 \\
23.6 \\
23.9 \\
24.1\end{array}$ & $\begin{array}{l}15.0 \\
15.1 \\
15.2 \\
15.3 \\
15.5 \\
15.8 \\
15.9\end{array}$ & $\begin{array}{l}8.6 \\
8.7 \\
8.7 \\
8.7 \\
8.8 \\
9.0 \\
9.1\end{array}$ \\
\hline $\begin{array}{l}\text { England and Wales } \\
1981 \\
1986 \\
1991 \\
1996\end{array}$ & $\begin{array}{l}71.0 \\
72.1 \\
73.4 \\
74.5\end{array}$ & $\begin{array}{l}67.1 \\
68.0 \\
69.1 \\
70.1\end{array}$ & $\begin{array}{l}52.5 \\
53.4 \\
54.4 \\
55.3\end{array}$ & $\begin{array}{l}42.9 \\
43.8 \\
44.8 \\
45.8\end{array}$ & $\begin{array}{l}24.3 \\
25.0 \\
26.1 \\
27.1\end{array}$ & $\begin{array}{l}16.4 \\
16.9 \\
17.8 \\
18.6\end{array}$ & $\begin{array}{l}10.1 \\
10.5 \\
11.2 \\
11.6\end{array}$ & $\begin{array}{l}5.8 \\
6.1 \\
6.4 \\
6.6\end{array}$ & $\begin{array}{l}1981 \\
1986 \\
1991 \\
1996\end{array}$ & $\begin{array}{l}77.0 \\
77.9 \\
78.9 \\
79.6\end{array}$ & $\begin{array}{l}72.9 \\
73.6 \\
74.5 \\
75.1\end{array}$ & $\begin{array}{l}58.1 \\
58.8 \\
59.7 \\
60.2\end{array}$ & $\begin{array}{l}48.3 \\
49.0 \\
49.9 \\
50.4\end{array}$ & $\begin{array}{l}29.4 \\
30.0 \\
30.8 \\
31.3\end{array}$ & $\begin{array}{l}20.9 \\
21.4 \\
22.0 \\
22.5\end{array}$ & $\begin{array}{l}13.4 \\
13.9 \\
14.4 \\
14.6\end{array}$ & $\begin{array}{l}7.5 \\
7.9 \\
8.3 \\
8.4\end{array}$ \\
\hline $\begin{array}{l}2000 \\
2001 \\
2002 \\
2003 \\
2004 \\
2005 \\
2006\end{array}$ & $\begin{array}{l}75.6 \\
75.9 \\
76.1 \\
76.4 \\
76.8 \\
77.2 \\
77.4\end{array}$ & $\begin{array}{l}71.1 \\
71.4 \\
71.6 \\
71.9 \\
72.3 \\
72.7 \\
72.9\end{array}$ & $\begin{array}{l}56.4 \\
56.7 \\
56.9 \\
57.2 \\
57.5 \\
57.9 \\
58.2\end{array}$ & $\begin{array}{l}46.8 \\
47.1 \\
47.3 \\
47.6 \\
47.9 \\
48.3 \\
48.5\end{array}$ & $\begin{array}{l}28.1 \\
28.4 \\
28.6 \\
28.9 \\
29.2 \\
29.6 \\
29.8\end{array}$ & $\begin{array}{l}19.6 \\
19.9 \\
20.1 \\
20.3 \\
20.6 \\
21.0 \\
21.2\end{array}$ & $\begin{array}{l}12.3 \\
12.5 \\
12.7 \\
12.9 \\
13.2 \\
13.5 \\
13.7\end{array}$ & $\begin{array}{l}7.0 \\
7.1 \\
7.2 \\
7.3 \\
7.4 \\
7.6 \\
7.7\end{array}$ & $\begin{array}{l}2000 \\
2001 \\
2002 \\
2003 \\
2004 \\
2005 \\
2006\end{array}$ & $\begin{array}{l}80.3 \\
80.5 \\
80.7 \\
80.9 \\
81.1 \\
81.5 \\
81.7\end{array}$ & $\begin{array}{l}75.8 \\
76.0 \\
76.1 \\
76.3 \\
76.6 \\
76.9 \\
77.1\end{array}$ & $\begin{array}{l}60.9 \\
61.2 \\
61.3 \\
61.5 \\
61.7 \\
62.0 \\
62.2\end{array}$ & $\begin{array}{l}51.1 \\
51.3 \\
51.5 \\
51.7 \\
51.9 \\
52.2 \\
52.4\end{array}$ & $\begin{array}{l}32.0 \\
32.2 \\
32.3 \\
32.5 \\
32.7 \\
33.1 \\
33.2\end{array}$ & $\begin{array}{l}23.1 \\
23.3 \\
23.4 \\
23.6 \\
23.8 \\
24.1 \\
24.3\end{array}$ & $\begin{array}{l}15.1 \\
15.2 \\
15.3 \\
15.4 \\
15.6 \\
15.9 \\
16.0\end{array}$ & $\begin{array}{l}8.6 \\
8.7 \\
8.7 \\
8.8 \\
8.9 \\
9.1 \\
9.2\end{array}$ \\
\hline $\begin{array}{l}\text { England } \\
1981 \\
1986 \\
1991 \\
1996\end{array}$ & $\begin{array}{l}71.1 \\
72.2 \\
73.4 \\
74.5\end{array}$ & $\begin{array}{l}67.1 \\
68.1 \\
69.1 \\
70.1\end{array}$ & $\begin{array}{l}52.5 \\
53.4 \\
54.4 \\
55.4\end{array}$ & $\begin{array}{l}42.9 \\
43.8 \\
44.9 \\
45.8\end{array}$ & $\begin{array}{l}24.3 \\
25.1 \\
26.2 \\
27.1\end{array}$ & $\begin{array}{l}16.4 \\
17.0 \\
17.8 \\
18.7\end{array}$ & $\begin{array}{l}10.1 \\
10.6 \\
11.2 \\
11.7\end{array}$ & $\begin{array}{l}5.8 \\
6.1 \\
6.4 \\
6.6\end{array}$ & $\begin{array}{l}1981 \\
1986 \\
1991 \\
1996\end{array}$ & $\begin{array}{l}77.0 \\
77.9 \\
78.9 \\
79.6\end{array}$ & $\begin{array}{l}72.9 \\
73.6 \\
74.5 \\
75.1\end{array}$ & $\begin{array}{l}58.2 \\
58.8 \\
59.7 \\
60.3\end{array}$ & $\begin{array}{l}48.4 \\
49.0 \\
49.9 \\
50.4\end{array}$ & $\begin{array}{l}29.4 \\
30.0 \\
30.8 \\
31.3\end{array}$ & $\begin{array}{l}20.9 \\
21.4 \\
22.0 \\
22.5\end{array}$ & $\begin{array}{l}13.4 \\
13.9 \\
14.4 \\
14.6\end{array}$ & $\begin{array}{l}7.5 \\
7.9 \\
8.3 \\
8.4\end{array}$ \\
\hline $\begin{array}{l}2000 \\
2001 \\
2002 \\
2003 \\
2004 \\
2005 \\
2006\end{array}$ & $\begin{array}{l}75.6 \\
75.9 \\
76.1 \\
76.5 \\
76.8 \\
77.2 \\
77.5\end{array}$ & $\begin{array}{l}71.2 \\
71.4 \\
71.7 \\
72.0 \\
72.3 \\
72.7 \\
73.0\end{array}$ & $\begin{array}{l}56.4 \\
56.7 \\
56.9 \\
57.2 \\
57.6 \\
57.9 \\
58.2\end{array}$ & $\begin{array}{l}46.9 \\
47.1 \\
47.4 \\
47.6 \\
48.0 \\
48.3 \\
48.6\end{array}$ & $\begin{array}{l}28.2 \\
28.5 \\
28.7 \\
28.9 \\
29.2 \\
29.6 \\
29.8\end{array}$ & $\begin{array}{l}19.6 \\
19.9 \\
20.1 \\
20.4 \\
20.7 \\
21.0 \\
21.2\end{array}$ & $\begin{array}{l}12.4 \\
12.6 \\
12.7 \\
12.9 \\
13.2 \\
13.5 \\
13.7\end{array}$ & $\begin{array}{l}7.0 \\
7.1 \\
7.2 \\
7.3 \\
7.4 \\
7.6 \\
7.7\end{array}$ & $\begin{array}{l}2000 \\
2001 \\
2002 \\
2003 \\
2004 \\
2005 \\
2006\end{array}$ & $\begin{array}{l}80.3 \\
80.6 \\
80.7 \\
80.9 \\
81.1 \\
81.5 \\
81.7\end{array}$ & $\begin{array}{l}75.8 \\
76.0 \\
76.1 \\
76.4 \\
76.6 \\
76.9 \\
77.1\end{array}$ & $\begin{array}{l}61.0 \\
61.2 \\
61.3 \\
61.5 \\
61.7 \\
62.1 \\
62.3\end{array}$ & $\begin{array}{l}51.2 \\
51.4 \\
51.5 \\
51.7 \\
51.9 \\
52.3 \\
52.4\end{array}$ & $\begin{array}{l}32.0 \\
32.2 \\
32.4 \\
32.6 \\
32.8 \\
33.1 \\
33.3\end{array}$ & $\begin{array}{l}23.1 \\
23.3 \\
23.4 \\
23.6 \\
23.8 \\
24.1 \\
24.3\end{array}$ & $\begin{array}{l}15.1 \\
15.2 \\
15.3 \\
15.4 \\
15.6 \\
15.9 \\
16.0\end{array}$ & $\begin{array}{l}8.6 \\
8.7 \\
8.7 \\
8.8 \\
8.9 \\
9.1 \\
9.2\end{array}$ \\
\hline $\begin{array}{l}\text { Wales } \\
1981 \\
1986 \\
1991 \\
1996\end{array}$ & $\begin{array}{l}70.4 \\
71.6 \\
73.1 \\
73.8\end{array}$ & $\begin{array}{l}66.5 \\
67.5 \\
68.8 \\
69.4\end{array}$ & $\begin{array}{l}51.9 \\
52.8 \\
54.1 \\
54.7\end{array}$ & $\begin{array}{l}42.2 \\
43.2 \\
44.6 \\
45.3\end{array}$ & $\begin{array}{l}23.6 \\
24.6 \\
25.8 \\
26.6\end{array}$ & $\begin{array}{l}15.8 \\
16.6 \\
17.6 \\
18.2\end{array}$ & $\begin{array}{c}9.7 \\
10.3 \\
11.0 \\
11.3\end{array}$ & $\begin{array}{l}5.6 \\
6.0 \\
6.4 \\
6.4\end{array}$ & $\begin{array}{l}1981 \\
1986 \\
1991 \\
1996\end{array}$ & $\begin{array}{l}76.4 \\
77.5 \\
78.8 \\
79.1\end{array}$ & $\begin{array}{l}72.3 \\
73.3 \\
74.3 \\
74.6\end{array}$ & $\begin{array}{l}57.5 \\
58.5 \\
59.5 \\
59.7\end{array}$ & $\begin{array}{l}47.7 \\
48.7 \\
49.7 \\
49.9\end{array}$ & $\begin{array}{l}28.9 \\
29.7 \\
30.6 \\
30.9\end{array}$ & $\begin{array}{l}20.5 \\
21.1 \\
21.8 \\
22.1\end{array}$ & $\begin{array}{l}13.1 \\
13.7 \\
14.3 \\
14.4\end{array}$ & $\begin{array}{l}7.4 \\
7.8 \\
8.3 \\
8.3\end{array}$ \\
\hline $\begin{array}{l}2000 \\
2001 \\
2002 \\
2003 \\
2004 \\
2005 \\
2006\end{array}$ & $\begin{array}{l}74.8 \\
75.3 \\
75.5 \\
75.8 \\
76.1 \\
76.6 \\
76.7\end{array}$ & $\begin{array}{l}70.4 \\
70.8 \\
70.9 \\
71.2 \\
71.6 \\
72.0 \\
72.1\end{array}$ & $\begin{array}{l}55.7 \\
56.0 \\
56.2 \\
56.5 \\
56.8 \\
57.3 \\
57.4\end{array}$ & $\begin{array}{l}46.2 \\
46.6 \\
46.8 \\
47.0 \\
47.3 \\
47.7 \\
47.8\end{array}$ & $\begin{array}{l}27.6 \\
28.0 \\
28.2 \\
28.4 \\
28.7 \\
29.2 \\
29.3\end{array}$ & $\begin{array}{l}19.1 \\
19.5 \\
19.7 \\
19.9 \\
20.2 \\
20.6 \\
20.8\end{array}$ & $\begin{array}{l}12.0 \\
12.3 \\
12.4 \\
12.6 \\
12.8 \\
13.2 \\
13.4\end{array}$ & $\begin{array}{l}6.8 \\
7.0 \\
7.1 \\
7.2 \\
7.3 \\
7.6 \\
7.6\end{array}$ & $\begin{array}{l}2000 \\
2001 \\
2002 \\
2003 \\
2004 \\
2005 \\
2006\end{array}$ & $\begin{array}{l}79.7 \\
80.0 \\
80.1 \\
80.3 \\
80.6 \\
80.9 \\
81.1\end{array}$ & $\begin{array}{l}75.2 \\
75.4 \\
75.5 \\
75.7 \\
76.0 \\
76.3 \\
76.5\end{array}$ & $\begin{array}{l}60.4 \\
60.6 \\
60.7 \\
60.9 \\
61.1 \\
61.5 \\
61.6\end{array}$ & $\begin{array}{l}50.6 \\
50.8 \\
50.9 \\
51.1 \\
51.3 \\
51.6 \\
51.8\end{array}$ & $\begin{array}{l}31.5 \\
31.7 \\
31.8 \\
32.0 \\
32.2 \\
32.6 \\
32.7\end{array}$ & $\begin{array}{l}22.6 \\
22.8 \\
22.9 \\
23.1 \\
23.3 \\
23.7 \\
23.8\end{array}$ & $\begin{array}{l}14.7 \\
14.9 \\
15.0 \\
15.1 \\
15.2 \\
15.5 \\
15.7\end{array}$ & $\begin{array}{l}8.4 \\
8.5 \\
8.6 \\
8.6 \\
8.7 \\
8.9 \\
9.0\end{array}$ \\
\hline $\begin{array}{l}\text { Scotland } \\
1981 \\
1986 \\
1991 \\
1996\end{array}$ & $\begin{array}{l}69.1 \\
70.2 \\
71.4 \\
72.2\end{array}$ & $\begin{array}{l}65.2 \\
66.0 \\
67.1 \\
67.8\end{array}$ & $\begin{array}{l}50.6 \\
51.4 \\
52.5 \\
53.1\end{array}$ & $\begin{array}{l}41.1 \\
41.9 \\
43.0 \\
43.7\end{array}$ & $\begin{array}{l}22.9 \\
23.5 \\
24.6 \\
25.3\end{array}$ & $\begin{array}{l}15.4 \\
15.8 \\
16.6 \\
17.3\end{array}$ & $\begin{array}{c}9.6 \\
9.9 \\
10.4 \\
10.9\end{array}$ & $\begin{array}{l}5.5 \\
5.7 \\
6.1 \\
6.3\end{array}$ & $\begin{array}{l}1981 \\
1986 \\
1991 \\
1996\end{array}$ & $\begin{array}{l}75.3 \\
76.2 \\
77.1 \\
77.9\end{array}$ & $\begin{array}{l}71.2 \\
71.9 \\
72.7 \\
73.3\end{array}$ & $\begin{array}{l}56.4 \\
57.1 \\
57.9 \\
58.5\end{array}$ & $\begin{array}{l}46.7 \\
47.3 \\
48.1 \\
48.8\end{array}$ & $\begin{array}{l}27.9 \\
28.4 \\
29.2 \\
29.8\end{array}$ & $\begin{array}{l}19.7 \\
20.1 \\
20.7 \\
21.2\end{array}$ & $\begin{array}{l}12.7 \\
13.0 \\
13.5 \\
13.8\end{array}$ & $\begin{array}{l}7.2 \\
7.5 \\
7.9 \\
8.0\end{array}$ \\
\hline $\begin{array}{l}2000 \\
2001 \\
2002 \\
2003 \\
2004 \\
2005 \\
2006\end{array}$ & $\begin{array}{l}73.1 \\
73.3 \\
73.5 \\
73.8 \\
74.2 \\
74.6 \\
74.8\end{array}$ & $\begin{array}{l}68.6 \\
68.8 \\
69.0 \\
69.3 \\
69.7 \\
70.1 \\
70.3\end{array}$ & $\begin{array}{l}53.9 \\
54.2 \\
54.3 \\
54.6 \\
55.0 \\
55.4 \\
55.5\end{array}$ & $\begin{array}{l}44.6 \\
44.8 \\
45.0 \\
45.2 \\
45.6 \\
45.9 \\
46.1\end{array}$ & $\begin{array}{l}26.3 \\
26.6 \\
26.7 \\
27.0 \\
27.3 \\
27.7 \\
27.9\end{array}$ & $\begin{array}{l}18.2 \\
18.4 \\
18.6 \\
18.8 \\
19.1 \\
19.4 \\
19.6\end{array}$ & $\begin{array}{l}11.5 \\
11.7 \\
11.8 \\
12.0 \\
12.2 \\
12.5 \\
12.6\end{array}$ & $\begin{array}{l}6.6 \\
6.8 \\
6.8 \\
6.9 \\
7.0 \\
7.2 \\
7.2\end{array}$ & $\begin{array}{l}2000 \\
2001 \\
2002 \\
2003 \\
2004 \\
2005 \\
2006\end{array}$ & $\begin{array}{l}78.6 \\
78.8 \\
78.9 \\
79.1 \\
79.3 \\
79.6 \\
79.7\end{array}$ & $\begin{array}{l}74.0 \\
74.2 \\
74.3 \\
74.5 \\
74.7 \\
75.0 \\
75.1\end{array}$ & $\begin{array}{l}59.2 \\
59.4 \\
59.5 \\
59.7 \\
59.9 \\
60.2 \\
60.3\end{array}$ & $\begin{array}{l}49.4 \\
49.6 \\
49.7 \\
49.9 \\
50.1 \\
50.4 \\
50.5\end{array}$ & $\begin{array}{l}30.5 \\
30.7 \\
30.8 \\
30.9 \\
31.1 \\
31.4 \\
31.5\end{array}$ & $\begin{array}{l}21.8 \\
22.0 \\
22.1 \\
22.2 \\
22.4 \\
22.7 \\
22.8\end{array}$ & $\begin{array}{l}14.1 \\
14.3 \\
14.4 \\
14.5 \\
14.7 \\
14.9 \\
15.0\end{array}$ & $\begin{array}{l}8.1 \\
8.2 \\
8.2 \\
8.3 \\
8.4 \\
8.5 \\
8.6\end{array}$ \\
\hline $\begin{array}{l}\text { Northern Ireland } \\
1981 \\
1986 \\
1991 \\
1996\end{array}$ & $\begin{array}{l}69.2 \\
70.9 \\
72.6 \\
73.8\end{array}$ & $\begin{array}{l}65.4 \\
66.8 \\
68.2 \\
69.4\end{array}$ & $\begin{array}{l}50.9 \\
52.2 \\
53.6 \\
54.7\end{array}$ & $\begin{array}{l}41.5 \\
42.7 \\
44.1 \\
45.3\end{array}$ & $\begin{array}{l}23.2 \\
24.2 \\
25.5 \\
26.6\end{array}$ & $\begin{array}{l}15.6 \\
16.4 \\
17.3 \\
18.2\end{array}$ & $\begin{array}{l}9.7 \\
10.4 \\
11.0 \\
11.4\end{array}$ & $\begin{array}{l}5.8 \\
6.2 \\
6.4 \\
6.6\end{array}$ & $\begin{array}{l}1981 \\
1986 \\
1991 \\
1996\end{array}$ & $\begin{array}{l}75.5 \\
77.1 \\
78.4 \\
79.2\end{array}$ & $\begin{array}{l}71.6 \\
72.9 \\
74.0 \\
74.7\end{array}$ & $\begin{array}{l}56.8 \\
58.1 \\
59.2 \\
59.9\end{array}$ & $\begin{array}{l}47.1 \\
48.3 \\
49.4 \\
50.0\end{array}$ & $\begin{array}{l}28.3 \\
29.3 \\
30.3 \\
30.9\end{array}$ & $\begin{array}{l}20.0 \\
20.8 \\
21.6 \\
22.1\end{array}$ & $\begin{array}{l}12.8 \\
13.4 \\
14.2 \\
14.4\end{array}$ & $\begin{array}{l}7.3 \\
7.8 \\
8.3 \\
8.4\end{array}$ \\
\hline $\begin{array}{l}2000 \\
2001 \\
2002 \\
2003 \\
2004 \\
2005 \\
2006\end{array}$ & $\begin{array}{l}74.8 \\
75.2 \\
75.6 \\
75.8 \\
76.0 \\
76.1 \\
76.2\end{array}$ & $\begin{array}{l}70.4 \\
70.7 \\
71.1 \\
71.4 \\
71.6 \\
71.6 \\
71.7\end{array}$ & $\begin{array}{l}55.7 \\
56.1 \\
56.4 \\
56.7 \\
56.9 \\
57.0 \\
57.1\end{array}$ & $\begin{array}{l}46.2 \\
46.6 \\
46.9 \\
47.1 \\
47.4 \\
47.5 \\
47.6\end{array}$ & $\begin{array}{l}27.6 \\
27.9 \\
28.2 \\
28.4 \\
28.7 \\
28.9 \\
29.1\end{array}$ & $\begin{array}{l}19.1 \\
19.4 \\
19.7 \\
19.9 \\
20.2 \\
20.4 \\
20.6\end{array}$ & $\begin{array}{l}11.9 \\
12.3 \\
12.4 \\
12.6 \\
12.8 \\
13.0 \\
13.1\end{array}$ & $\begin{array}{l}6.6 \\
6.9 \\
7.0 \\
7.2 \\
7.3 \\
7.3 \\
7.3\end{array}$ & $\begin{array}{l}2000 \\
2001 \\
2002 \\
2003 \\
2004 \\
2005 \\
2006\end{array}$ & $\begin{array}{l}79.8 \\
80.1 \\
80.4 \\
80.6 \\
80.8 \\
81.0 \\
81.2\end{array}$ & $\begin{array}{l}75.2 \\
75.6 \\
75.9 \\
76.0 \\
76.3 \\
76.4 \\
76.6\end{array}$ & $\begin{array}{l}60.4 \\
60.7 \\
61.0 \\
61.1 \\
61.4 \\
61.6 \\
61.8\end{array}$ & $\begin{array}{l}50.6 \\
50.9 \\
51.2 \\
51.3 \\
51.6 \\
51.8 \\
52.0\end{array}$ & $\begin{array}{l}31.5 \\
31.8 \\
32.0 \\
32.2 \\
32.5 \\
32.7 \\
32.8\end{array}$ & $\begin{array}{l}22.6 \\
22.9 \\
23.1 \\
23.3 \\
23.5 \\
23.7 \\
23.9\end{array}$ & $\begin{array}{l}14.6 \\
14.9 \\
15.1 \\
15.2 \\
15.4 \\
15.6 \\
15.7\end{array}$ & $\begin{array}{l}8.2 \\
8.4 \\
8.5 \\
8.6 \\
8.7 \\
8.8 \\
8.9\end{array}$ \\
\hline
\end{tabular}

Note: All figures are based on a three-year period, so that for instance 2003 represents 2002-2004. The population estimates used to calculate these life expectancies are the latest available at time of publication of the 2005-2007 interim life tables (30 October 2008). All figures are based on death registrations. A minor methodological change was introduced for the 2006 figures to ensure consistency with population estimates of the very elderly for England and Wales. The effect on calculated life expectancies is marginal. 


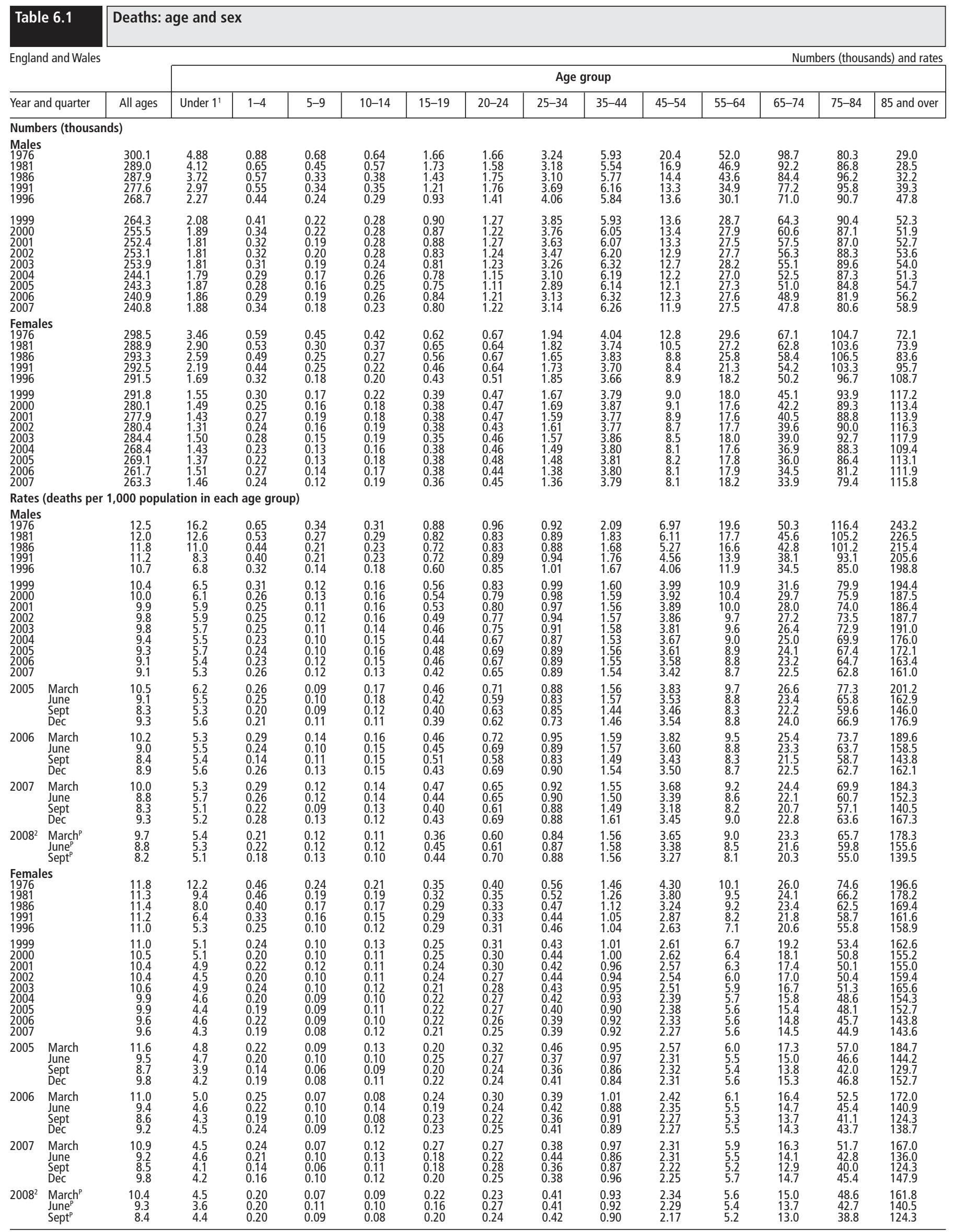

Note: Figures represent the numbers of deaths registered in each year up to 1992 and the numbers of deaths occurring in each year from 1993 to $2005.2006,2007$ and provisional 2008 figures relate to registrations.

2 Death rates for 2008 are based on the 2006-based population projections for 2008.

p provisional. 


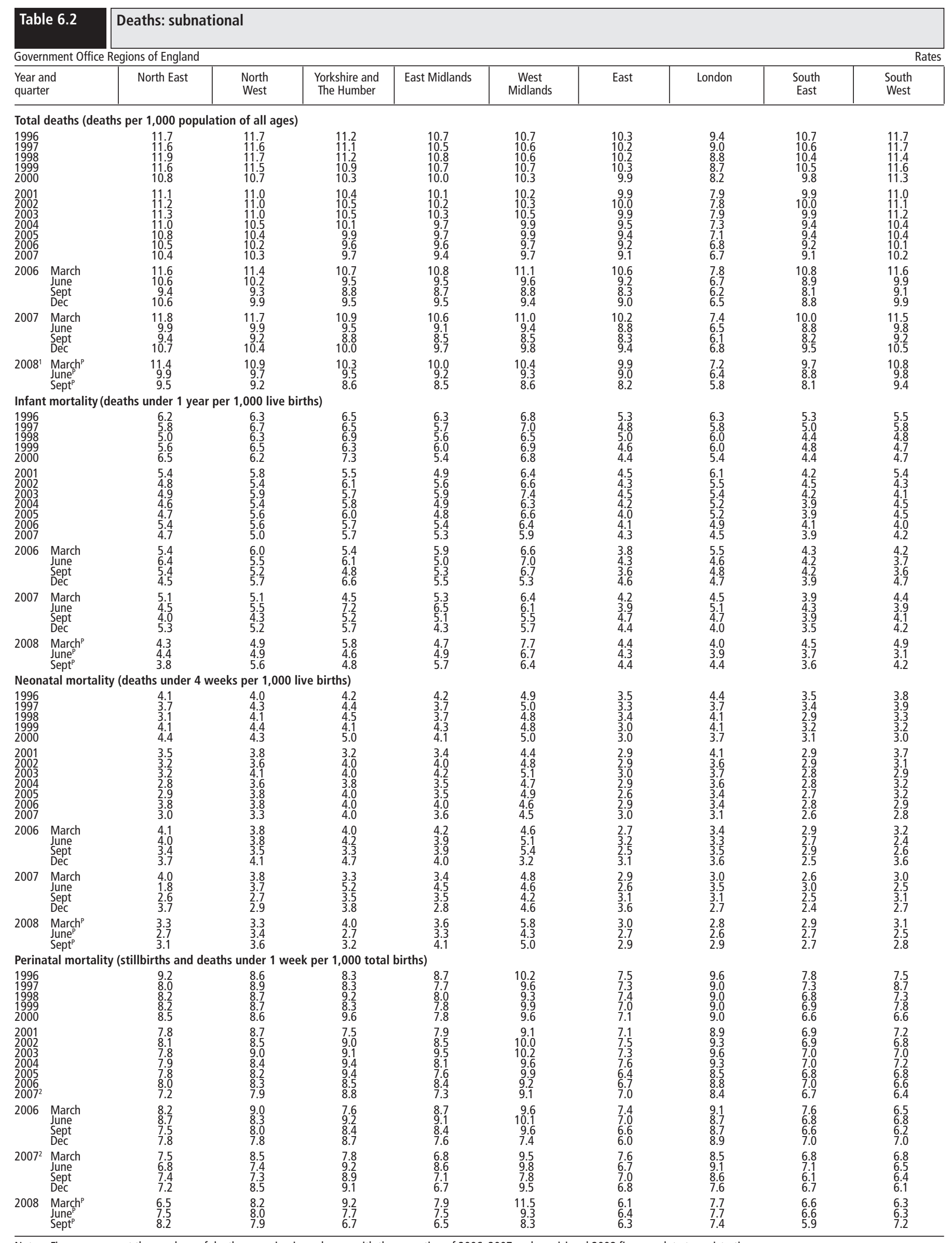

Note: Figures represent the numbers of deaths occurring in each year with the exception of 2006, 2007 and provisional 2008 figures relate to registrations. 1 Total deaths rates for 2008 have been calculated using the mid-2007 population estimates published on 21 August 2008.

22007 Perinatal figures have been updated to include late receipt of stillbirth records.

p provisional. 
Number (thousands) and rate for all deaths and age-standardised rates per million population for selected causes

\begin{tabular}{|c|c|c|c|c|c|c|c|c|c|c|c|c|c|}
\hline \multirow{3}{*}{$\begin{array}{l}\text { Year and } \\
\text { quarter }\end{array}$} & & & & & & & & Malignant ne & plasms & & & & \\
\hline & \multicolumn{2}{|c|}{ All deaths } & \multirow{2}{*}{$\begin{array}{l}\text { All causes } \\
\text { (age - } \\
\text { standardised } \\
\text { rates per } \\
\text { million } \\
\text { population²) }\end{array}$} & \multirow[t]{2}{*}{ Oesophagus } & \multirow[t]{2}{*}{ Stomach } & \multirow[t]{2}{*}{ Colon } & \multirow{2}{*}{$\begin{array}{l}\text { Rectosigmoid } \\
\text { junction, } \\
\text { rectum, and } \\
\text { anus }\end{array}$} & \multirow{2}{*}{$\begin{array}{l}\text { Trachea, } \\
\text { bronchus } \\
\text { and lung }\end{array}$} & \multirow{2}{*}{$\begin{array}{c}\text { Melanoma } \\
\text { of skin }\end{array}$} & \multirow{2}{*}{$\begin{array}{c}\text { Other } \\
\text { malignant } \\
\text { neoplasms } \\
\text { of skin }\end{array}$} & \multirow[t]{2}{*}{ Breast } & \multirow{2}{*}{$\begin{array}{l}\text { Cervix } \\
\text { uteri }\end{array}$} & \multirow[t]{2}{*}{ Ovary } \\
\hline & $\begin{array}{l}\text { Number } \\
\text { (thousands) }\end{array}$ & $\begin{array}{c}\text { Crude } \\
\text { rate per } \\
100,000 \\
\text { population }\end{array}$ & & & & & & & & & & & \\
\hline & & & $\begin{array}{l}\text { A00-R99 } \\
\text { V01-Y89 }\end{array}$ & (C15) & (C16) & (C18) & (C19-C21) & (С33-C34) & (C43) & (C44) & (C50) & (C53) & (C56) \\
\hline
\end{tabular}

Males

$1971 \quad 288.4 \quad 1,207 \quad 13,466$

$\begin{array}{llll}1981 & 288.4 & 1,207 & 13,466 \\ 1991 & 289.0 & 1,196 & 12,189\end{array}$

$1991 \quad 277.6 \quad 1,125 \quad 10,291$

$\begin{array}{llll}1998 & 264.7 & 1,064 & 8,981 \\ 1999 & 264.3 & 1,044 & 8,862\end{array}$

$\begin{array}{llll}1999 & 264.3 & 1,044 & 8,862 \\ 2000 & 255.5 & 1,005 & 8,437\end{array}$

$\begin{array}{rrrr}2001 & 252.4 & 987 & 8,188\end{array}$

$\begin{array}{llll}2002 & 253.1 & 985 & 8,081\end{array}$

$\begin{array}{llll}2003 & 253.9 & 982 & 8,000\end{array}$

$2005-243.3 \quad 929$

$2006 \quad 240.9 \quad 913 \quad 7,35$

$2007 \quad 240.9$

2006 March $\quad 66.5 \quad 1,023$

June

Sept

Dec

2007 March

Sept

Dec

62.0

June

Sept

Females

$1971 \quad 278.9 \quad 1,104 \quad 8,189$

$\begin{array}{llll}1981 & 288.9 & 1,134 & 8,189 \\ 199 & 292.5 & 1,122 & 6,425\end{array}$

$1991 \quad 292.5 \quad 1,122$

$\begin{array}{llll}1998 & 290.3 & 1,108 & 5,945\end{array}$

$\begin{array}{llll}1999 & 291.8 & 1,097 & 5,929 \\ 2000 & 280.1 & 1,049 & 5,655\end{array}$

$\begin{array}{llll}2001 & 280.1 & 1,049 & 5,655 \\ 2002 & 280.4 & 1,038 & 5,543\end{array}$

$\begin{array}{llll}2002 & 280.4 & 1,043 & 5,524\end{array}$

$2003 \quad 284.4 \quad 1,055 \quad 5,575$

$\begin{array}{rrrr}2004 & 268.4 & 1,075 & 5,206 \\ 2005 & 269.4 & 990 & 5,188\end{array}$

$\begin{array}{llll}2005 & 269.4 & 990 & 5,188 \\ 2006 & 261.7 & 956 & 4,989\end{array}$

$\begin{array}{llll}2006 & 261.7 & 956 & 4,989 \\ 2007 & 263.3 & 957 & 4,921\end{array}$

2006 March $\quad 74.5 \quad 1,104 \quad 5,658$

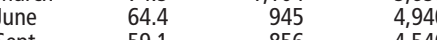

$\begin{array}{llll}\text { Sept } & 59.1 & 856 & 4,540 \\ \text { Dec } & 63.7 & 923 & 4,832\end{array}$

2007 March $\quad 74.0 \quad 1,091 \quad 5,523$

$\begin{array}{lrrr}\text { June } & 62.8 & 916 & 4,757 \\ \text { Sept } & 58.8 & 847 & 4,408\end{array}$

$\begin{array}{llll}\text { Dec } & 67.7 & 976 & 4,408 \\ & 67.8 & & 5167\end{array}$

$2008^{3}$ March $\quad 71.7^{\mathrm{P}} \quad 1,042^{\mathrm{p}} \quad 5,252^{\mathrm{p}}$

\begin{tabular}{lrrr} 
June & $63.8^{\mathrm{P}}$ & $927^{\mathrm{P}}$ & $4,758^{\mathrm{P}}$ \\
Sept & $58.8^{\mathrm{P}}$ & $844^{\mathrm{P}}$ & $4,402^{\mathrm{P}}$ \\
\hline
\end{tabular}

4,402

$\begin{array}{rrrrrrrr}76 & 317 & 187 & 144 & 1,066 & 10 & 12 & 4 \\ 90 & 251 & 181 & 135 & 1,028 & 17 & 9 & 3 \\ 117 & 185 & 194 & 117 & 842 & 23 & 10 & \\ 129 & 132 & 169 & 95 & 643 & 26 & 8 & 3 \\ 127 & 127 & 161 & 90 & 611 & 27 & 7 & 2 \\ 128 & 118 & 158 & 89 & 592 & 28 & 7 & 2 \\ 129 & 111 & 155 & 89 & 570 & 26 & 7 & 3 \\ 131 & 110 & 151 & 90 & 559 & 27 & 8 & 3 \\ 135 & 102 & 145 & 90 & 539 & 28 & 8 & 2 \\ 129 & 95 & 143 & 92 & 521 & 30 & 9 & 2 \\ 132 & 93 & 137 & 92 & 515 & 28 & 8 & \\ 131 & 83 & 132 & 90 & 509 & 31 & 7 & 2 \\ 128 & 82 & 128 & 88 & 498 & 31 & 8 & 3 \\ 131 & 82 & 134 & 98 & 522 & 32 & 7 & 2 \\ 132 & 82 & 128 & 87 & 504 & 30 & 7 & 2 \\ 128 & 81 & 133 & 85 & 497 & 29 & 7 & 1 \\ 131 & 86 & 133 & 91 & 515 & 31 & 8 & 3 \\ 126 & 88 & 132 & 86 & 523 & 33 & 7 & 2 \\ 130 & 84 & 122 & 86 & 493 & 31 & 8 & 3 \\ 128 & 80 & 126 & 87 & 470 & 29 & 7 & 3 \\ 129 & 78 & 132 & 94 & 509 & 31 & 9 & 3\end{array}$

$135^{\mathrm{P}} \quad 80^{\mathrm{P}} \quad 132^{\mathrm{P}}$

$\begin{array}{lllll}125^{\mathrm{P}} & 77^{\mathrm{P}} & 130^{\mathrm{P}} & 90^{\mathrm{P}} & 476^{\mathrm{P}} \\ 126^{\mathrm{P}} & 79^{\mathrm{P}} & 129^{\mathrm{P}} & 91^{\mathrm{P}} & 483\end{array}$

$29^{\mathrm{p}}$
$33^{\mathrm{P}}$
$30^{\mathrm{P}}$

$\begin{array}{ll}5^{p} & 1^{p} \\ 8^{p} & 2^{p} \\ 8^{p} & 2^{p}\end{array}$

$\begin{array}{rrrrrrrrrr}40 & 149 & 176 & 79 & 183 & 14 & 6 & 379 & 83 & 126 \\ 42 & 111 & 157 & 74 & 252 & 16 & 5 & 405 & 69 & 121 \\ 50 & 74 & 146 & 61 & 300 & 18 & 4 & 401 & 54 & 118 \\ 49 & 54 & 117 & 47 & 291 & 21 & 3 & 328 & 35 & 116 \\ 52 & 51 & 115 & 46 & 289 & 20 & 3 & 319 & 33 & 111 \\ 51 & 48 & 107 & 45 & 285 & 21 & 3 & 311 & 33 & 109 \\ 48 & 46 & 103 & 45 & 283 & 20 & 3 & 308 & 31 & 112 \\ 51 & 44 & 103 & 44 & 284 & 19 & 3 & 302 & 29 & 112 \\ 50 & 42 & 98 & 46 & 285 & 20 & 3 & 293 & 27 & 108 \\ 48 & 41 & 96 & 46 & 283 & 19 & 3 & 278 & 26 & 100 \\ 48 & 39 & 96 & 46 & 290 & 21 & 3 & 284 & 26 & 102 \\ 48 & 35 & 93 & 46 & 300 & 19 & 4 & 277 & 24 & 99 \\ 47 & 35 & 92 & 48 & 300 & 21 & 4 & 267 & 24 & 96 \\ 48 & 40 & 90 & 45 & 309 & 16 & 4 & 296 & 26 & 105 \\ 46 & 34 & 89 & 46 & 294 & 18 & 4 & 266 & 22 & 101 \\ 47 & 33 & 99 & 44 & 289 & 19 & 3 & 272 & 23 & 96 \\ 51 & 34 & 95 & 49 & 307 & 21 & 4 & 273 & 23 & 93 \\ 50 & 35 & 92 & 49 & 315 & 22 & 4 & 284 & 25 & 95 \\ 48 & 31 & 88 & 45 & 296 & 22 & 4 & 267 & 22 & 97 \\ 40 & 37 & 91 & 47 & 285 & 21 & 3 & 251 & 22 & 97 \\ 51 & 37 & 96 & 49 & 306 & 20 & 4 & 269 & 25 & 94\end{array}$

\begin{tabular}{ll}
$50^{\mathrm{P}}$ & $35^{\mathrm{P}}$ \\
$46^{\mathrm{P}}$ & $36^{\mathrm{P}}$ \\
\hline
\end{tabular}

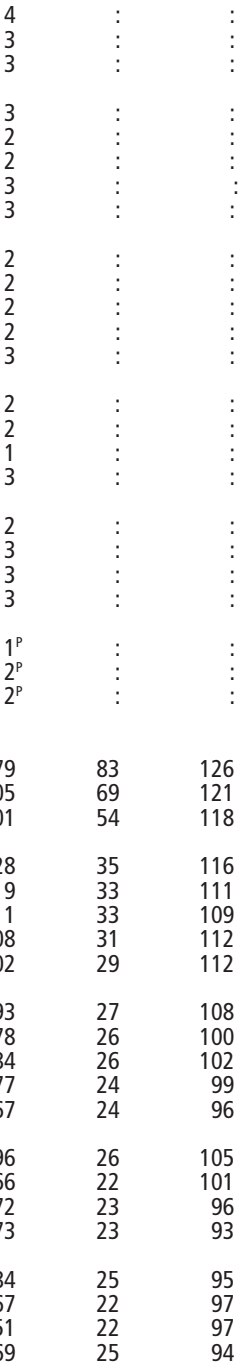

$\begin{array}{lll}269^{\mathrm{P}} & 24^{\mathrm{P}} & 93^{\mathrm{P}} \\ 259^{\mathrm{P}} & 23^{\mathrm{P}} & 91^{\mathrm{P}} \\ 253^{\mathrm{P}} & 23^{\mathrm{P}} & 92^{\mathrm{P}}\end{array}$

Note: Figures represent the number of deaths registered in each year up to 1992 and the number of deaths occurring in each year from 1993 to 2005 . The number of deaths for 2006 onwards relate to registrations.

The rates by cause of death in this table are based on final underlying cause. For further details see the Explanatory Notes in the 'Report: Death registrations in England and Wales, 2004: causes' in $\mathrm{HSQ} 26$.

Death rates from 2002 to 2005 have been updated to include the latest revised mid-year population estimates that take into account improved estimates of international migration.

1 The Ninth Revision of the International Classification of Diseases, 1975, came into operation in England and Wales on 1 January 1979. The Tenth Revision of the International Classification of

Diseases, 1992, came into operation in England and Wales on 1 January 2001. The cause descriptions and codes relate to ICD-10. For changes to this table see 'In Brief', Health Statistics Quarterly 14.

Dopulation. See Notes to Tables.

Death rates for 2008 are provisional and based on the 2006-based population projections for 2008

provisional. 
Age-standardised rates ${ }^{2}$ per million population for selected causes Malignant neoplasms

\begin{tabular}{|c|c|c|c|c|c|c|c|c|c|c|c|c|c|}
\hline Prostate & Bladder & Leukaemia & $\begin{array}{l}\text { Diabetes } \\
\text { mellitus }\end{array}$ & $\begin{array}{l}\text { Ischaemic } \\
\text { heart } \\
\text { disease }\end{array}$ & $\begin{array}{l}\text { Cerebro } \\
\text { vascular } \\
\text { diseases }\end{array}$ & Pneumonia & $\begin{array}{l}\text { Bronchitis, } \\
\text { emphysema } \\
\text { and other } \\
\text { chronic } \\
\text { obstructive } \\
\text { pulmonary } \\
\text { disease }\end{array}$ & Asthma & $\begin{array}{c}\text { Gastric } \\
\text { and } \\
\text { duodenal } \\
\text { ulcer }\end{array}$ & $\begin{array}{l}\text { Diseases } \\
\text { of the liver }\end{array}$ & $\begin{array}{c}\text { Land } \\
\text { transport } \\
\text { accidents }\end{array}$ & $\begin{array}{l}\text { Intentional } \\
\text { self-harm and } \\
\text { events of } \\
\text { undetermined } \\
\text { intent }\end{array}$ & $\begin{array}{c}\text { Year and } \\
\text { quarter }\end{array}$ \\
\hline (C61) & (C67) & (C91-C95) & (E10-E14) & $(120-125)$ & (160-169) & (J12-J18) & (J40-J44) & (J45-J46) & (K25-K27) & (K70-K76) & (V01-V89) & $\begin{array}{l}\text { (X60-X84, } \\
\text { Y10-Y34) }\end{array}$ & \\
\hline $\begin{array}{l}198 \\
214 \\
304\end{array}$ & $\begin{array}{l}124 \\
121 \\
121\end{array}$ & $\begin{array}{l}74 \\
74 \\
77\end{array}$ & $\begin{array}{r}82 \\
82 \\
131\end{array}$ & $\begin{array}{l}3,801 \\
3,664 \\
2,984\end{array}$ & $\begin{array}{r}1,541 \\
1,141 \\
940\end{array}$ & $\begin{array}{r}920 \\
1,053 \\
391\end{array}$ & $\begin{array}{l}944 \\
683 \\
606\end{array}$ & $\begin{array}{l}21 \\
28 \\
31\end{array}$ & $\begin{array}{r}107 \\
90 \\
73\end{array}$ & $\begin{array}{l}41 \\
58 \\
76\end{array}$ & $\begin{array}{l}209 \\
119 \\
125\end{array}$ & $\begin{array}{l}124 \\
151 \\
160\end{array}$ & $\begin{array}{r}\text { Males } \\
1971 \\
1981 \\
1991\end{array}$ \\
\hline $\begin{array}{l}277 \\
272 \\
260 \\
274 \\
271\end{array}$ & $\begin{array}{l}99 \\
93 \\
92 \\
93 \\
90\end{array}$ & $\begin{array}{l}67 \\
67 \\
67 \\
70 \\
68\end{array}$ & $\begin{array}{l}94 \\
94 \\
88 \\
94 \\
91\end{array}$ & $\begin{array}{l}2,215 \\
2,095 \\
1,959 \\
1,872 \\
1,784\end{array}$ & $\begin{array}{l}706 \\
673 \\
622 \\
690 \\
690\end{array}$ & $\begin{array}{l}720 \\
770 \\
735 \\
388 \\
388\end{array}$ & $\begin{array}{l}463 \\
474 \\
416 \\
403 \\
396\end{array}$ & $\begin{array}{l}18 \\
18 \\
17 \\
16 \\
15\end{array}$ & $\begin{array}{l}60 \\
64 \\
59 \\
55 \\
56\end{array}$ & $\begin{array}{l}115 \\
119 \\
119 \\
139 \\
144\end{array}$ & $\begin{array}{l}86 \\
86 \\
86 \\
86 \\
83\end{array}$ & $\begin{array}{l}152 \\
151 \\
141 \\
134 \\
131\end{array}$ & $\begin{array}{l}1998 \\
1999 \\
2000 \\
2001 \\
2002\end{array}$ \\
\hline $\begin{array}{l}273 \\
267 \\
256 \\
250 \\
247\end{array}$ & $\begin{array}{l}87 \\
85 \\
80 \\
81 \\
81\end{array}$ & $\begin{array}{l}71 \\
67 \\
67 \\
68 \\
66\end{array}$ & $\begin{array}{l}91 \\
83 \\
79 \\
74 \\
71\end{array}$ & $\begin{array}{l}1,703 \\
1,566 \\
1,470 \\
1,353 \\
1,280\end{array}$ & $\begin{array}{l}662 \\
595 \\
555 \\
520 \\
481\end{array}$ & $\begin{array}{l}408 \\
360 \\
353 \\
320 \\
303\end{array}$ & $\begin{array}{l}411 \\
364 \\
368 \\
343 \\
337\end{array}$ & $\begin{array}{l}14 \\
15 \\
12 \\
10 \\
11\end{array}$ & $\begin{array}{l}53 \\
50 \\
46 \\
45 \\
39\end{array}$ & $\begin{array}{l}157 \\
151 \\
156 \\
161 \\
162\end{array}$ & $\begin{array}{l}84 \\
77 \\
75 \\
83 \\
79\end{array}$ & $\begin{array}{l}129 \\
125 \\
118 \\
123 \\
119\end{array}$ & $\begin{array}{l}2003 \\
2004 \\
2005 \\
2006 \\
2007\end{array}$ \\
\hline $\begin{array}{l}256 \\
249 \\
241 \\
252\end{array}$ & $\begin{array}{l}79 \\
81 \\
83 \\
80\end{array}$ & $\begin{array}{l}73 \\
63 \\
67 \\
69\end{array}$ & $\begin{array}{l}86 \\
75 \\
66 \\
71\end{array}$ & $\begin{array}{l}1,543 \\
1,351 \\
1,210 \\
1,312\end{array}$ & $\begin{array}{l}611 \\
506 \\
454 \\
509\end{array}$ & $\begin{array}{l}434 \\
318 \\
242 \\
287\end{array}$ & $\begin{array}{l}440 \\
351 \\
271 \\
312\end{array}$ & $\begin{array}{r}11 \\
10 \\
11 \\
8\end{array}$ & $\begin{array}{l}52 \\
48 \\
41 \\
41\end{array}$ & $\begin{array}{l}158 \\
164 \\
158 \\
164\end{array}$ & $\begin{array}{l}83 \\
90 \\
77 \\
82\end{array}$ & $\begin{array}{l}128 \\
117 \\
112 \\
134\end{array}$ & $\begin{array}{r}2006 \text { March } \\
\text { June } \\
\text { Sept } \\
\text { Dec }\end{array}$ \\
\hline $\begin{array}{l}252 \\
246 \\
233 \\
257\end{array}$ & $\begin{array}{l}83 \\
80 \\
78 \\
82\end{array}$ & $\begin{array}{l}66 \\
69 \\
61 \\
69\end{array}$ & $\begin{array}{l}77 \\
65 \\
65 \\
75\end{array}$ & $\begin{array}{l}1,442 \\
1,249 \\
1,146 \\
1,287\end{array}$ & $\begin{array}{l}541 \\
465 \\
429 \\
489\end{array}$ & $\begin{array}{l}395 \\
286 \\
226 \\
306\end{array}$ & $\begin{array}{l}433 \\
311 \\
265 \\
339\end{array}$ & $\begin{array}{r}11 \\
11 \\
9 \\
11\end{array}$ & $\begin{array}{l}44 \\
37 \\
35 \\
40\end{array}$ & $\begin{array}{l}178 \\
154 \\
152 \\
167\end{array}$ & $\begin{array}{l}84 \\
77 \\
73 \\
82\end{array}$ & $\begin{array}{l}115 \\
121 \\
116 \\
125\end{array}$ & $\begin{array}{r}2007 \text { March } \\
\text { June } \\
\text { Sept } \\
\text { Dec }\end{array}$ \\
\hline $\begin{array}{l}239^{p} \\
228^{p} \\
238^{p}\end{array}$ & $\begin{array}{l}75^{p} \\
78^{p} \\
76^{p}\end{array}$ & $\begin{array}{l}64^{p} \\
65^{p} \\
61^{p}\end{array}$ & $\begin{array}{l}75^{p} \\
72^{p} \\
69^{p}\end{array}$ & $\begin{array}{l}1,324^{\mathrm{P}} \\
1,191^{\mathrm{P}} \\
1,069^{\mathrm{P}}\end{array}$ & $\begin{array}{l}514^{p} \\
453^{p} \\
409^{p}\end{array}$ & $\begin{array}{l}363^{p} \\
283^{p} \\
225^{p}\end{array}$ & $\begin{array}{l}412^{\mathrm{P}} \\
321^{\mathrm{P}} \\
260^{\mathrm{P}}\end{array}$ & $\begin{array}{r}11^{\mathrm{P}} \\
8^{\mathrm{P}} \\
8^{\mathrm{P}}\end{array}$ & $\begin{array}{l}47^{p} \\
39^{p} \\
34^{p}\end{array}$ & $\begin{array}{l}164^{\mathrm{p}} \\
160^{\mathrm{p}} \\
161^{\mathrm{P}}\end{array}$ & $\begin{array}{l}67^{p} \\
70^{p} \\
66^{p}\end{array}$ & $\begin{array}{l}107^{\mathrm{p}} \\
121^{\mathrm{p}} \\
137^{\mathrm{P}}\end{array}$ & $\begin{array}{r}2008 \text { March } \\
\text { June } \\
\text { Sept }\end{array}$ \\
\hline $\begin{array}{l}\vdots \\
\vdots \\
\vdots\end{array}$ & $\begin{array}{l}32 \\
35 \\
34\end{array}$ & $\begin{array}{l}47 \\
47 \\
44\end{array}$ & $\begin{array}{l}89 \\
66 \\
95\end{array}$ & $\begin{array}{l}1,668 \\
1,601 \\
1,407\end{array}$ & $\begin{array}{r}1,352 \\
1,012 \\
812\end{array}$ & $\begin{array}{l}624 \\
740 \\
325\end{array}$ & $\begin{array}{l}193 \\
155 \\
211\end{array}$ & $\begin{array}{l}25 \\
30 \\
30\end{array}$ & $\begin{array}{l}44 \\
57 \\
46\end{array}$ & $\begin{array}{l}31 \\
43 \\
49\end{array}$ & $\begin{array}{l}82 \\
41 \\
45\end{array}$ & $\begin{array}{l}84 \\
81 \\
51\end{array}$ & $\begin{array}{r}\text { Females } \\
1971 \\
1981 \\
1991\end{array}$ \\
\hline $\begin{array}{l}\vdots \\
\vdots \\
\vdots\end{array}$ & $\begin{array}{l}32 \\
30 \\
31 \\
29 \\
30\end{array}$ & $\begin{array}{l}41 \\
45 \\
39 \\
41 \\
43\end{array}$ & $\begin{array}{l}65 \\
65 \\
62 \\
62 \\
65\end{array}$ & $\begin{array}{r}1,055 \\
986 \\
907 \\
878 \\
843\end{array}$ & $\begin{array}{l}645 \\
629 \\
577 \\
620 \\
616\end{array}$ & $\begin{array}{l}546 \\
591 \\
546 \\
307 \\
316\end{array}$ & $\begin{array}{l}226 \\
241 \\
216 \\
220 \\
224\end{array}$ & $\begin{array}{l}22 \\
22 \\
20 \\
19 \\
20\end{array}$ & $\begin{array}{l}41 \\
39 \\
41 \\
39 \\
37\end{array}$ & $\begin{array}{l}64 \\
67 \\
68 \\
77 \\
79\end{array}$ & $\begin{array}{l}28 \\
28 \\
24 \\
23 \\
24\end{array}$ & $\begin{array}{l}43 \\
45 \\
45 \\
40 \\
41\end{array}$ & $\begin{array}{l}1998 \\
1999 \\
2000 \\
2001 \\
2002\end{array}$ \\
\hline $\begin{array}{l}\vdots \\
\vdots \\
\vdots\end{array}$ & $\begin{array}{l}30 \\
28 \\
28 \\
29 \\
27\end{array}$ & $\begin{array}{l}39 \\
39 \\
39 \\
36 \\
38\end{array}$ & $\begin{array}{l}66 \\
60 \\
57 \\
54 \\
53\end{array}$ & $\begin{array}{l}811 \\
736 \\
686 \\
629 \\
592\end{array}$ & $\begin{array}{l}606 \\
548 \\
519 \\
478 \\
455\end{array}$ & $\begin{array}{l}337 \\
296 \\
298 \\
261 \\
251\end{array}$ & $\begin{array}{l}244 \\
214 \\
224 \\
213 \\
218\end{array}$ & $\begin{array}{l}20 \\
17 \\
17 \\
16 \\
14\end{array}$ & $\begin{array}{l}36 \\
35 \\
32 \\
29 \\
26\end{array}$ & $\begin{array}{l}81 \\
78 \\
81 \\
87 \\
87\end{array}$ & $\begin{array}{l}24 \\
20 \\
22 \\
24 \\
23\end{array}$ & $\begin{array}{l}41 \\
38 \\
38 \\
39 \\
35\end{array}$ & $\begin{array}{l}2003 \\
2004 \\
2005 \\
2006 \\
2007\end{array}$ \\
\hline $\begin{array}{l}\vdots \\
\vdots \\
\vdots\end{array}$ & $\begin{array}{l}29 \\
27 \\
29 \\
29\end{array}$ & $\begin{array}{l}42 \\
34 \\
35 \\
35\end{array}$ & $\begin{array}{l}60 \\
56 \\
51 \\
51\end{array}$ & $\begin{array}{l}733 \\
637 \\
562 \\
585\end{array}$ & $\begin{array}{l}551 \\
477 \\
427 \\
459\end{array}$ & $\begin{array}{l}371 \\
259 \\
186 \\
231\end{array}$ & $\begin{array}{l}283 \\
214 \\
163 \\
193\end{array}$ & $\begin{array}{l}19 \\
16 \\
13 \\
16\end{array}$ & $\begin{array}{l}37 \\
27 \\
27 \\
25\end{array}$ & $\begin{array}{l}87 \\
85 \\
86 \\
89\end{array}$ & $\begin{array}{l}25 \\
27 \\
21 \\
23\end{array}$ & $\begin{array}{l}40 \\
37 \\
41 \\
38\end{array}$ & $\begin{array}{r}2006 \text { March } \\
\text { June } \\
\text { Sept } \\
\text { Dec }\end{array}$ \\
\hline $\begin{array}{l}\vdots \\
\vdots \\
\vdots\end{array}$ & $\begin{array}{l}29 \\
29 \\
24 \\
26\end{array}$ & $\begin{array}{l}40 \\
36 \\
34 \\
41\end{array}$ & $\begin{array}{l}58 \\
49 \\
50 \\
56\end{array}$ & $\begin{array}{l}689 \\
569 \\
520 \\
591\end{array}$ & $\begin{array}{l}517 \\
439 \\
399 \\
464\end{array}$ & $\begin{array}{l}353 \\
228 \\
178 \\
249\end{array}$ & $\begin{array}{l}298 \\
204 \\
156 \\
217\end{array}$ & $\begin{array}{l}18 \\
14 \\
12 \\
14\end{array}$ & $\begin{array}{l}28 \\
27 \\
24 \\
26\end{array}$ & $\begin{array}{l}96 \\
86 \\
80 \\
86\end{array}$ & $\begin{array}{l}23 \\
27 \\
22 \\
20\end{array}$ & $\begin{array}{l}33 \\
31 \\
35 \\
39\end{array}$ & $\begin{array}{r}2007 \text { March } \\
\text { June } \\
\text { Sept } \\
\text { Dec }\end{array}$ \\
\hline $\begin{array}{l}\vdots \\
\vdots\end{array}$ & $\begin{array}{l}29^{p} \\
27^{p} \\
25^{p}\end{array}$ & $\begin{array}{l}36^{p} \\
36^{p} \\
36^{p}\end{array}$ & $\begin{array}{l}54^{\mathrm{P}} \\
51^{\mathrm{p}} \\
46^{\mathrm{P}}\end{array}$ & $\begin{array}{l}613^{p} \\
547^{p} \\
499^{p}\end{array}$ & $\begin{array}{l}487^{p} \\
432^{p} \\
381^{p}\end{array}$ & $\begin{array}{l}320^{p} \\
237^{p} \\
170^{p}\end{array}$ & $\begin{array}{l}262^{p} \\
206^{p} \\
159^{p}\end{array}$ & $\begin{array}{l}17^{p} \\
14^{p} \\
11^{p}\end{array}$ & $\begin{array}{l}28^{p} \\
26^{p} \\
27^{p}\end{array}$ & $\begin{array}{l}90^{p} \\
85^{p} \\
78^{p}\end{array}$ & $\begin{array}{l}20^{p} \\
22^{p} \\
28^{p}\end{array}$ & $\begin{array}{l}33^{p} \\
39^{p} \\
40^{p}\end{array}$ & $\begin{array}{r}2008^{3} \text { March } \\
\text { June } \\
\text { Sept }\end{array}$ \\
\hline
\end{tabular}

